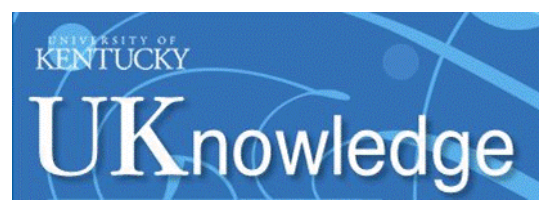

University of Kentucky

UKnowledge

$11-29-2016$

\title{
SDSS-IV MaNGA IFS Galaxy Survey-Survey Design, Execution, and Initial Data Quality
}

\author{
Renbin Yan \\ University of Kentucky, yanrenbin@uky.edu \\ Kevin Bundy \\ The University of Tokyo, Japan \\ David R. Law \\ Space Telescope Science Institute \\ Matthew A. Bershady \\ University of Wisconsin - Madison \\ Brett Andrews \\ University of Pittsburgh \\ Follow this and additional works at: https://uknowledge.uky.edu/physastron_facpub \\ Part of the Astrophysics and Astronomy Commons, and the Physics Commons \\ See next page for additional authors \\ Right click to open a feedback form in a new tab to let us know how this document benefits you.
}

\section{Repository Citation}

Yan, Renbin; Bundy, Kevin; Law, David R.; Bershady, Matthew A.; Andrews, Brett; Cherinka, Brian; DiamondStanic, Aleksandar M.; Drory, Niv; MacDonald, Nicholas; Sánchez-Gallego, José R.; Thomas, Daniel; Wake, David A.; Weijmans, Anne-Marie; Westfall, Kyle B.; Zhang, Kai; Aragón-Salamanca, Alfonso; Belfiore, Francesco; Bizyaev, Dmitry; Blanc, Guillermo A.; Blanton, Michael R.; Brownstein, Joel; Cappellari, Michele; D'Souza, Richard; Emsellem, Eric; Fu, Hai; Gaulme, Patrick; Graham, Mark T.; Goddard, Daniel; Gunn, James E.; and Harding, Paul, "SDSS-IV MaNGA IFS Galaxy Survey-Survey Design, Execution, and Initial Data Quality" (2016). Physics and Astronomy Faculty Publications. 497.

https://uknowledge.uky.edu/physastron_facpub/497

This Article is brought to you for free and open access by the Physics and Astronomy at UKnowledge. It has been accepted for inclusion in Physics and Astronomy Faculty Publications by an authorized administrator of UKnowledge. For more information, please contact UKnowledge@lsv.uky.edu. 


\title{
SDSS-IV MaNGA IFS Galaxy Survey-Survey Design, Execution, and Initial Data Quality
}

\author{
Digital Object Identifier (DOI) \\ https://doi.org/10.3847/0004-6256/152/6/197
}

\section{Notes/Citation Information}

Published in The Astronomical Journal, v. 152, no. 6, 197, p. 1-32.

(C) 2016. The American Astronomical Society. All rights reserved.

The copyright holder has granted the permission for posting the article here.

Due to the large number of authors, only the first 30 and the authors affiliated with the University of Kentucky are listed in the author section above. For the complete list of authors, please download this article.

\section{Authors}

Renbin Yan, Kevin Bundy, David R. Law, Matthew A. Bershady, Brett Andrews, Brian Cherinka, Aleksandar M. Diamond-Stanic, Niv Drory, Nicholas MacDonald, José R. Sánchez-Gallego, Daniel Thomas, David A. Wake, Anne-Marie Weijmans, Kyle B. Westfall, Kai Zhang, Alfonso Aragón-Salamanca, Francesco Belfiore, Dmitry Bizyaev, Guillermo A. Blanc, Michael R. Blanton, Joel Brownstein, Michele Cappellari, Richard D'Souza, Eric Emsellem, Hai Fu, Patrick Gaulme, Mark T. Graham, Daniel Goddard, James E. Gunn, and Paul Harding 


\title{
SDSS-IV MaNGA IFS GALAXY SURVEY—SURVEY DESIGN, EXECUTION, AND INITIAL DATA QUALITY
}

\author{
Renbin Yan ${ }^{1}$, Kevin Bundy ${ }^{2}$, David R. Law ${ }^{3}$, Matthew A. Bershady ${ }^{4}$, Brett Andrews ${ }^{5}$, Brian Cherinka ${ }^{6}$, \\ Aleksandar M. Diamond-Stanic ${ }^{4}$, Niv Drory ${ }^{7}$, Nicholas MacDonald ${ }^{8}$, José R. Sánchez-Gallego ${ }^{8}$, Daniel Thomas ${ }^{9,38}$, \\ David A. Wake ${ }^{10}$, Anne-Marie Weijmans ${ }^{11}$, Kyle B. Westfall ${ }^{9,38}$, Kai Zhang $^{1}$, Alfonso Aragón-Salamanca ${ }^{12}$, \\ Francesco Belfiore ${ }^{13,14}$, Dmitry Bizyaev ${ }^{15,16}$, Guillermo A. Blanc ${ }^{17,18,39}{ }^{2}$, Michael R. Blanton ${ }^{19}$, Joel Brownstein ${ }^{20}$, \\ Michele Cappellari $^{21}{ }^{2}$, Richard D’Souza ${ }^{22}$, Eric Emsellem ${ }^{23,24,25}$, Hai Fu ${ }^{26}$, Patrick Gaulme ${ }^{15}$, Mark T. Graham ${ }^{21}$, \\ Daniel Goddard ${ }^{9,38}$, James E. Gunn ${ }^{27}$, Paul Harding ${ }^{28}$, Amy Jones ${ }^{22}$, Karen Kinemuchi ${ }^{15}$, Cheng Li $^{29,30}$, HongYu Li $^{31,32}$, \\ Roberto Maiolino $^{13,14}$, Shude Mao ${ }^{30,31,33}$, Claudia Maraston ${ }^{9,38}$, Karen Masters ${ }^{9,38}$, Michael R. Merrifield ${ }^{12}$, \\ Daniel Oravetz ${ }^{15}$, Kaike Pan ${ }^{15}$, John K. Parejko ${ }^{8}$, Sebastian F. Sanchez ${ }^{34}$, David Schlegel ${ }^{35}$, Audrey Simmons ${ }^{15}$, \\ Karun Thanjavur $^{36}$, Jeremy Tinker ${ }^{19}$, Christy Tremonti ${ }^{4}$, Remco van den Bosch ${ }^{37}$, and Zheng Zheng ${ }^{31}$ \\ ${ }^{1}$ Department of Physics and Astronomy, University of Kentucky, 505 Rose Street, \\ Lexington, KY 40506-0057, USA; yanrenbin@uky.edu \\ ${ }^{2}$ Kavli IPMU (WPI), UTIAS, The University of Tokyo, Kashiwa, Chiba 277-8583, Japan \\ ${ }^{3}$ Space Telescope Science Institute, 3700 San Martin Drive, Baltimore, MD 21218, USA \\ ${ }^{4}$ Department of Astronomy, University of Winsconsin-Madison, 475 N. Charter Street, Madison, WI 53706-1582, USA \\ ${ }^{5}$ Department of Physics and Astronomy and Pittsburgh Particle Physics, Astrophysics and Cosmology Center (PITT PACC), \\ University of Pittsburgh, 3941 OHara Street, Pittsburgh, PA 15260, USA \\ ${ }^{6}$ Department of Physics and Astronomy, Johns Hopkins University, Bloomberg Center, \\ 3400 N. Charles Street, Baltimore, MD 21218, USA \\ ${ }^{7}$ McDonald Observatory, University of Texas at Austin, 1 University Station, Austin, TX 78712-0259, USA \\ ${ }^{8}$ Department of Astronomy, Box 351580, University of Washington, Seattle, WA 98195, USA \\ ${ }^{9}$ Institute of Cosmology and Gravitation, University of Portsmouth, Portsmouth, UK \\ ${ }^{10}$ Department of Physical Sciences, The Open University, Milton Keynes, MK7 6AA, UK \\ ${ }^{11}$ School of Physics and Astronomy, University of St Andrews, North Haugh, St Andrews KY16 9SS, UK \\ ${ }^{12}$ School of Physics and Astronomy, University of Nottingham, University Park, Nottingham NG7 2RD, UK \\ ${ }_{14}^{13}$ Cavendish Laboratory, University of Cambridge, 19 J. J. Thomson Avenue, Cambridge CB3 OHE, UK \\ ${ }_{15}^{14}$ Kavli Institute for Cosmology, University of Cambridge, Madingley Road, CB3 OHA Cambridge, UK \\ 15 Apache Point Observatory and New Mexico State University, P.O. Box 59, Sunspot, NM 88349, USA \\ ${ }^{16}$ Sternberg Astronomical Institute, Moscow State University, Universitetskij pr. 13, Moscow, Russia \\ ${ }^{17}$ Departamento de Astronoma, Universidad de Chile, Camino el Observatorio 1515, Las Condes, Santiago, Chile \\ ${ }^{18}$ Centro de Astrofsica y Tecnologas Afines (CATA), Camino del Observatorio 1515, Las Condes, Santiago, Chile \\ ${ }^{19}$ Center for Cosmology and Particle Physics, Department of Physics, \\ New York University, 4 Washington Place, NY 10003, USA \\ ${ }^{20}$ Department of Physics and Astronomy, University of Utah, 115 S 1400 E, Salt Lake City, UT 84112, USA \\ ${ }^{21}$ Sub-Department of Astrophysics, Department of Physics, University of Oxford, \\ DenysWilkinson Building, Keble Road, Oxford OX1 3RH, UK \\ ${ }^{22}$ Max Plank Institute for Astrophysics, Munich, D-85741, Garching, Germany \\ ${ }^{23}$ European Southern Observatory, Karl-Schwarzschild-Strasse 2, D-85748 Garching, Germany \\ ${ }^{24}$ Université Lyon 1, Observatoire de Lyon, Centre de Recherche Astrophysique de Lyon, \\ 9 avenue Charles André, F-69561 Saint Genis Laval Cedex, France
${ }^{25}$ Ecole Normale Supérieure de Lyon, 9 avenue Charles André, F-69230 Saint-Genis Laval, France \\ ${ }^{26}$ Department of Physics and Astronomy, 203 Van Allen Hall, University of Iowa, Iowa City, IA 52242-1479, USA \\ ${ }^{27}$ Department of Astrophysical Sciences, Princeton University, Princeton, NJ 08544, USA \\ ${ }^{28}$ Department of Astronomy, Case Western Reserve University, Cleveland, OH 44106, USA \\ ${ }^{29}$ Shanghai Astronomical Observatory, Nandan Road 80, Shanghai 200030, China \\ ${ }^{30}$ Department of Physics and Tsinghua Center for Astrophysics, Tsinghua University, Beijing 100086, China \\ ${ }^{31}$ National Astronomical Observatories, Chinese Academy of Sciences, 20A Datun Road, Chaoyang District, Beijing 100012, China \\ ${ }^{32}$ University of Chinese Academy of Sciences, Beijing 100049, China \\ ${ }^{33}$ Jodrell Bank Centre for Astrophysics, School of Physics and Astronomy, \\ The University of Manchester, Oxford Road, Manchester M13 9PL, UK \\ ${ }^{34}$ Instituto de Astronomia, Universidad Nacional Autonoma deMexico, A.P. 70-264, 04510 Mexico D.F., Mexico \\ ${ }_{36}^{35}$ Physics Division, Lawrence Berkeley National Laboratory, Berkeley, CA 94720-8160, USA \\ ${ }^{36}$ Department of Physics and Astronomy, University of Victoria, Victoria, BC V8P5C2, Canada \\ ${ }^{37}$ Max Planck Institute for Astronomy, Königstuhl 17, D-69117 Heidelberg, Germany \\ Received 2016 April 20; revised 2016 July 15; accepted 2016 July 24; published 2016 November 29
}

\begin{abstract}
The MaNGA Survey (Mapping Nearby Galaxies at Apache Point Observatory) is one of three core programs in the Sloan Digital Sky Survey IV. It is obtaining integral field spectroscopy for 10,000 nearby galaxies at a spectral resolution of $R \sim 2000$ from 3622 to $10354 \AA$. The design of the survey is driven by a set of science requirements on the precision of estimates of the following properties: star formation rate surface density, gas metallicity, stellar population age, metallicity, and abundance ratio, and their gradients; stellar and gas kinematics; and enclosed
\end{abstract}

\footnotetext{
38 SEPnet, South East Physics Network (http://www.sepnet.ac.uk).

39 Visiting Astronomer, Observatories of the Carnegie Institution for Science,

813 Santa Barbara Street, Pasadena, CA 91101, USA.
} 
gravitational mass as a function of radius. We describe how these science requirements set the depth of the observations and dictate sample selection. The majority of targeted galaxies are selected to ensure uniform spatial coverage in units of effective radius $\left(R_{e}\right)$ while maximizing spatial resolution. About two-thirds of the sample is covered out to $1.5 R_{e}$ (Primary sample), and one-third of the sample is covered to $2.5 R_{e}$ (Secondary sample). We describe the survey execution with details that would be useful in the design of similar future surveys. We also present statistics on the achieved data quality, specifically the point-spread function, sampling uniformity, spectral resolution, sky subtraction, and flux calibration. For our Primary sample, the median $r$-band signal-to-noise ratio is $\sim 70$ per $1.4 \AA$ pixel for spectra stacked between $1 R_{e}$ and $1.5 R_{e}$. Measurements of various galaxy properties from the first-year data show that we are meeting or exceeding the defined requirements for the majority of our science goals.

Key words: galaxies: evolution - galaxies: general - surveys - techniques: imaging spectroscopy

\section{INTRODUCTION}

Large spectroscopic galaxy surveys, such as the Sloan Digital Sky Survey (SDSS; York et al. 2000) and the Twodegree Field Galaxy Redshift Survey (Colless et al. 2001), have revolutionized the way we study galaxy evolution. The huge statistical power brought in by targeting a large number of galaxies using the same instrument with excellent calibration enabled huge progress. Not only have these efforts quantified accurately with great precision those trends and scaling relations that were previously known, such as the color bimodality (Strateva et al. 2001; Baldry et al. 2004), the color-density relation (Hogg et al. 2003; Blanton et al. 2005), the mass-metallicity relation for gas (Tremonti et al. 2004) and stars (Thomas et al. 2010; Johansson et al. 2012), and the fundamental plane (Bernardi et al. 2003), but they have also discovered many new relations and trends, such as the dependence of star formation history on stellar mass (Kauffmann et al. 2003), the star formation rate (SFR) versus stellar mass relation (Brinchmann et al. 2004; Salim et al. 2007; Wuyts et al. 2011), the strong mass dependence of the radioloud active galactic nucleus fraction (Best et al. 2005), largescale galactic conformity (Kauffmann et al. 2013), and many others. They also connected large-scale structure studies and galaxy evolution studies thanks to environmental measurements enabled by dense and uniform sampling of complete galaxy samples (see Blanton \& Moustakas 2009 and references therein).

However, these massive surveys lacked spatial coverage in individual galaxies. The single $3^{\prime \prime}$ fibers used by SDSS, for instance, cannot cover most of the light in nearby galaxies. For example, comparing the flux incident on the SDSS $3^{\prime \prime}$ fibers with the total flux of all main sample galaxies in SDSS, $80 \%$ of galaxies have less than $36 \%$ of their light covered by the fiber. The spectra provide a lot of information, about both stellar and gaseous components, but they only sample the center of the galaxies and can give a strongly biased picture. Nearly all studies based on SDSS have to take this aperture effect into account in their analysis. Many studies combining spectroscopic information with photometry also need to make corrections, make extrapolations, or use simplified assumptions. For example, to obtain the total SFR in a galaxy, one has to either apply large aperture corrections to the spectroscopically derived SFR based on the central region (Brinchmann et al. 2004) or turn to broadband photometry, which suffers more from dust extinction and degeneracies in stellar population modeling (Salim et al. 2005, 2007). Furthermore, a full kinematic description is impossible with single-fiber observations. Past long-slit surveys are also inefficient at obtaining the spatial information as one only probes a narrow elongated region and the signal-to-noise ratio $(\mathrm{S} / \mathrm{N})$ is poor in galaxy outskirts.

Integral field spectroscopy (IFS) solves these problems. Several IFS surveys have made great progress in recent years (see Cappellari 2016 for a review). SAURON (Bacon et al. 2001) and ATLAS3D (Cappellari et al. 2011) surveyed 260 early-type galaxies in the nearby universe using a lenslet array intergral field instrument, SAURON, on the William Herschel Telescope on La Palma. They had a relatively narrow wavelength coverage (4800-5380 $⿱$ ) and focused exclusively on early-type galaxies. The DiskMass survey (Bershady et al. 2010) used two fiber-fed integral field units (IFUs), SparsePak on WIYN and PPak on the Calar Alto $3.5 \mathrm{~m}$ telescope. It targeted 146 nearly face-on disk galaxies to study stellar and gas kinematics. For the purpose of kinematic measurements, this survey utilized high spectral resolution in three narrow wavelength windows around 515, 660, and $860 \mathrm{~nm}$. The VENGA (Blanc et al. 2013) survey used a fiberfed integral field spectrograph, VIRUS-P on the $2.7 \mathrm{~m}$ telescope at McDonald Observatory, and targeted 30 nearby spiral galaxies. Recently, the CALIFA survey used the PPak instrument and targeted 600 nearby galaxies selected to sample a wide variety of stellar mass and SFR. With the improved sample size and wide wavelength coverage, CALIFA has produced numerous results, such as the universal metallicity gradient among star-forming galaxies (Sánchez et al. 2014), the nature of LINER-like galaxies (Kehrig et al. 2012; Singh et al. 2013; Gomes et al. 2016), the spatially resolved growth history (Pérez et al. 2013; Sánchez-Blázquez et al. 2014), the spatially resolved stellar mass-metallicity relation (González Delgado et al. 2014), and the resolved star formation main sequence (Cano-Díaz et al. 2016; González Delgado et al. 2016). However, if one were to do an SDSS-like study of galaxies by binning galaxies by stellar mass, environment, and morphology, one quickly loses sample size for significant statistics (e.g., González Delgado et al. 2016). The main limitation for the sample size is that all these surveys are targeting galaxies one by one and are inefficient at building up a large statistical sample.

To address this issue, two large IFS surveys of the general galaxy population targeting thousands of galaxies are ongoing right now. Both utilize multiple fiber bundles to target multiple galaxies at the same time, enabling much more efficient observing. One of them is the SAMI Galaxy survey (Allen et al. 2015; Bryant et al. 2015) using the fiber-fed SAMI instrument (Croom et al. 2012) on the $3.5 \mathrm{~m}$ Anglo-Australian Telescope at Siding Spring Observatory. SAMI will eventually target 3400 galaxies and has already produced results on many topics, including the kinematic morphology-density relation 
(Fogarty et al. 2014), outflows and extraplanar gas (Ho et al. 2014, 2016), dynamical scaling relations (Cortese et al. 2014), dynamical mass-to-light ratio $(M / L)$ of disk galaxies (Cecil et al. 2016), and aperture corrections for SFRs (Richards et al. 2016). The other large IFS survey is the SDSSIV/MaNGA galaxy survey operating at the $2.5 \mathrm{~m}$ Sloan Foundation Telescope. Given the large $3^{\circ}$ field of view of the SDSS telescope and sizable detector real estate, MaNGA uses multiple fiber bundles to target 17 galaxies (and 12 standard stars) at the same time. This allows us to build a 10,000-galaxy IFS sample with much wider and continuous wavelength coverage than other surveys, enabling powerful statistical studies of the spatially resolved properties of nearby galaxies.

This paper complements MaNGA's other descriptive publications by providing a complete picture of the survey's design and execution and an evaluation of the resulting data quality. In Section 2, we describe the science requirements of our survey and how they flow down to specific decisions on the sample design and observing strategy. We summarize the hardware in Section 3 and the sample design in Section 4. In Section 5, we describe the execution of the survey, including the observing strategy, setting of the completeness thresholds, choice of the fields, plate design, observing procedure, and the optimization of the instrument focus. In Section 6 we describe our survey progress and projection. In Section 7, we provide an evaluation of the initial data quality: point-spread function (PSF), sampling uniformity, spectral resolution, sky subtraction accuracy, and flux calibration accuracy. In the end (Section 8), we present a series of tests checking whether we are meeting the science requirements. We summarize in Section 9.

\section{SCIENCE REQUIREMENTS}

In Bundy et al. (2015) we have described the scientific motivation of the survey. In this paper, we provide the specific requirements that drive the design. Bundy et al. (2015) listed four key science questions we aim to address: how galactic disks grow through accretion, how galactic bulges and ellipticals build up, how star formation shuts down by internal and/or external processes, and how mass and angular momentum are distributed in galaxies. In order to put the survey design on a quantitative footing, we need to turn these general questions into specific requirements that dictate design choices and can be verified with initial data. The seven key measurement requirements discussed below flow down from the key science questions.

In setting these requirements, we would like to be particularly pedantic about distinguishing precision from accuracy. "Precision" of a measurement is set by the random errors, while "accuracy" of a measurement is set by both random and systematic errors. Below, for direct observables that are independent of model assumptions, such as redshift, radial velocity, and velocity dispersion, we can set requirements on both precision and accuracy. But for derived quantities that are model dependent, such as SFR, stellar age, gas metallicity, dark matter fraction, etc., we only set requirements on the precision of the measurements, under a specific set of model assumptions. The model dependence of these quantities means that their accuracies completely depend on how accurate the models are, which is often difficult to assess. Investigating the accuracy of the models is an important goal for astronomy but is beyond the scope of our tasks of designing the survey.
In choosing the threshold values for each requirement, we generally follow the rule that we require the precision to be better than half of the $1 \sigma$ scatter in the expected distribution of the quantity. Now we describe the requirements:

1. We require the SFR surface density to be measured to a precision of 0.15 dex per spatial resolution element in our target galaxies where SFR surface density is higher than $0.01 M_{\odot} \mathrm{yr}^{-1} \mathrm{kpc}^{-2}$ and reddening $(E(B-V))$ is less than 0.5 .

SFR is an essential indicator of galaxy growth. It is required to address the questions of disk growth, bulge growth, and how star formation shuts down. With MaNGA, we measure the surface density of the SFR. The main estimator of SFR at our disposal is the extinction-corrected $\mathrm{H} \alpha$ luminosity, which traces the instantenous SFR and is often used as the basis for calibrating other SFR indicators (Kennicutt 1998; Kennicutt \& Evans 2012). The SFR in star-forming galaxies correlates tightly with stellar mass, which is often referred to as the star formation main sequence (Brinchmann et al. 2004; Salim et al. 2005; Noeske et al. 2007; Renzini $\&$ Peng 2015). The $1 \sigma$ scatter acround this relationship in the local universe is about 0.3 dex (Renzini \& Peng 2015), which includes both intrinsic scatter and measurement noise. With MaNGA data, we will measure the spatially resolved version of this relationship_-star formation surface density versus stellar mass surface density (Cano-Díaz et al. 2016). Thus, we require the precision on SFR surface density to be better than half of the scatter in the global relationship.

Of course, such precision can only be sensible above a certain threshold of emission-line strength. We set the threshold to the "knee" in the Schmidt-Kennicutt relation where the SFR efficiency changes significantly (Bigiel et al. 2008), which is around $0.01 M_{\odot} \mathrm{yr}^{-1} \mathrm{kpc}^{-2}$. In addition, extinction can severely affect our ability to measure SFR. We thus set a maximum extinction for our requirement, $E(B-V)=0.5$, which is about the median extinction in the centers of star-forming galaxies in the SDSS main sample.

2. Measurements of the gas metallicity gradients in galaxies should have a precision of 0.04 dex per $R_{e}$.

Gas metallicity gradients can provide crucial insights about the cycling of gas in galaxies. We will measure gas metallicity from strong emission lines. Supernova explosions and mass loss from asymptotic giant branch stars could return gas enriched with newly synthesized material. Feedback from star formation can drive gas enriched with heavy elements back into the hot halo, which could rain back down later when it cools. How the enriched gas is redistributed in the galaxy disk can be reflected by the radial metallicity gradient. Fu et al. (2012) used a radially resolved semianalytical model and demonstrated that the gradient is sensitive to the fraction of gas ejected into the hot halo. The higher the fraction of gas ejected into the halo, which eventually redistributes over the disk, the flatter is the metallicity gradient. The models also predict gradients to be a function of stellar mass and bulge-to-disk ratios. Observationally, recent work by Sánchez et al. (2014) showed a universal gradient of $-0.1 \mathrm{dex} / R_{e}$ with a sigma of $0.09 \mathrm{dex} / R_{e}$. In order to discern the potential 
dependence on mass and bulge fraction, we need to measure the gradient to at least a factor of 2 better than the scatter seen in CALIFA. Therefore, we chose 0.04 dex per $R_{e}$ as our requirement.

On the other hand, the behavior of the gradient at large radius could directly probe the gas accretion at large radius. Moran et al. (2012) found that $10 \%$ of massive disk galaxies show abrupt drops at large radius, while Sánchez et al. (2014) found flattening in metallicity at the same place. Resolving this apparent discrepancy requires a large sample size, wide stellar mass sampling, and sufficient precision to resolve the intrinsic scatter, which we expect will be no smaller than the scatter at small radii.

3. Measurements of light-weighted stellar age gradients in star-forming and newly quenched galaxies should have a precision of 0.1 dex per $R_{e}$.

We can measure light-weighted stellar age in starforming and newly quenched galaxies using D4000 and $\mathrm{H} \delta$ absorption indices, following Kauffmann et al. (2003). This will help address the question about bulge growth and about quenching. The average stellar age measurements are sensitive to the assumed stellar population models with many ingredients, including the initial mass function (IMF), the stellar spectral library, the template star formation history, and metallicity evolution (Chen et al. 2010). Therefore, we set our requirements on the precision of the measurement under a specific set of stellar population models. Recent work on CALIFA disk galaxies shows that gradients range from +0.4 to $-0.7 \mathrm{dex} / R_{e}$, with a $1 \sigma$ scatter of $0.2 \mathrm{dex} / R_{e}$ at fixed stellar masses, which is comparable to the scatter in stellar age itself (González Delgado et al. 2015). We set our requirements to half of the scatter.

4. Measurements of light-weighted stellar age, metallicity, and abundance gradients in quiescent galaxies should have a precision of 0.1 dex per decade in $R_{e}$.

In quiescent galaxies (galaxies without recent star formation), the stellar age, metallicity, and abundance can be measured from either absorption-line indices or full spectral fitting. Our precision requirement of 0.1 dex per decade in $R_{e}$ is chosen because typical age gradients range from -0.1 to 0.4 dex per decade in $R_{e}$ with a $1 \sigma$ scatter of about 0.2 dex (Mehlert et al. 2003; Kuntschner et al. 2010; Spolaor et al. 2010) and typical abundance gradients range from -0.5 to +0.2 dex per decade in $R_{e}$ with a scatter of about 0.2 dex (Mehlert et al. 2003; Spolaor et al. 2010; Koleva et al. 2011).

5. Measurements of the baryonic specific angular momentum $\left(\lambda_{R}\right)$ within $1 R_{e}$ should have an accuracy of 0.05 for $\lambda \sim 0.1$ for quiescent galaxies in order to differentiate fast and slow rotators, given that the dividing line between the two is roughly around $\lambda=0.1$ (Cappellari et al. 2007; Emsellem et al. 2007, 2011). Since the measurement of $\lambda$ is model independent, this is an accuracy requirement.

6. Measurements of the enclosed gravitating mass within $1.5 R_{e}$ should have an accuracy of $10 \%$ when the kinematics appear to be axisymmetric.

7. Measurements of the dark matter fraction within $1.5 R_{e}$ or $2.5 R_{e}$ should have a precision of $10 \%$ in bulge-dominated gas-poor galaxies. The measurement becomes more difficult for star-forming galaxies due to the larger uncertainty on stellar mass estimates from stellar population models. Thus, we only set the requirements for bulge-dominated gas-poor galaxies.

This is a precision requirement because the measurement is model dependent. The assumptions about the dark matter profile, the $M / L$, and its gradient will change the result systematically.

The last two requirements are set not only because they are interesting on their own but also by the desire to measure the stellar $M / L$ in bulge-dominated, gas-poor galaxies via dynamical modeling to $15 \%-25 \%$ to investigate IMF variations. This constraint is driven by the fact that the maximum $M / L$ difference attributed to the IMF is roughly a factor of 2 (e.g., Cappellari et al. 2012; Conroy \& van Dokkum 2012b).

In addition, we require measuring the dependence of all of the above physical properties on stellar mass, morphology, and environment. This is one of the major motivations to obtain a sample as large as MaNGA.

\subsection{Requirements on the Depth of the Observation}

The above requirements flow down to quantitative limits on our survey design.

1. With the calibration provided by Kennicutt \& Evans (2012), an SFR surface density of $0.01 M_{\odot} \mathrm{yr}^{-1} \mathrm{kpc}^{-2}$ corresponds to an extinction-corrected $\mathrm{H} \alpha$ surface brightness $(\mathrm{SB})$ of $1.86 \times 10^{39} \mathrm{erg} \mathrm{s}^{-1} \mathrm{kpc}^{-2}$. At a reddening of $E(B-V)=0.5$, the Balmer decrement is 4.85 and the observed $\mathrm{H} \alpha$ luminosity is $31.3 \%$ of the intrinsic $\mathrm{H} \alpha$ luminosity, corresponding to an $\mathrm{H} \alpha$ surface brightness of $5.83 \times 10^{38} \mathrm{erg} \mathrm{s}^{-1} \mathrm{kpc}^{-2}$. This is $1.15 \times 10^{-16} /$ $(1+z)^{4} \mathrm{erg} \mathrm{s}^{-1} \mathrm{~cm}^{-2} \operatorname{arcsec}^{-2}$ in observed surface brightness. Because of the surface brightness dimming with redshift, this requirement is most stringent at the high- $z$ limit of our sample. At $z \sim 0.15$, this corresponds to an $\mathrm{H} \alpha \mathrm{SB}$ of $6.58 \times 10^{-17} \mathrm{erg} \mathrm{s}^{-1} \mathrm{~cm}^{-2} \operatorname{arcsec}^{-2}$ and an $\mathrm{H} \beta \mathrm{SB}$ of $1.36 \times 10^{-17} \mathrm{erg} \mathrm{s}^{-1} \mathrm{~cm}^{-2} \operatorname{arcsec}^{-2}$.

To convert the measurement precision of SFR surface density to the required $\mathrm{S} / \mathrm{N}$ in the spectra, we need to consider how the SFR estimate is made. Both the uncertainty on the raw $\mathrm{H} \alpha$ measurement and the uncertainty on the extinction correction derived from the Balmer decrement $(\mathrm{H} \alpha / \mathrm{H} \beta$ ratio $)$ need to be considered. In Yan et al. (2016), we have done a detailed derivation of this dependence. According to Equation (12) in that paper, we find that the measurement noise on $\mathrm{H} \beta$ dominates the final error budget. At an extinction of $E$ $(B-V)=0.5, \mathrm{H} \alpha$ is 4.85 times as strong as $\mathrm{H} \beta$, and we assume that it has at least 3 times higher $\mathrm{S} / \mathrm{N}$ than $\mathrm{H} \beta$. In order to measure SFR to 0.15 dex or equivalently $(0.15 \mathrm{ln}$ $10=) 34.5 \%$ in fractional uncertainty, we need to measure $\mathrm{H} \alpha$ to better than $5 \%$ and $\mathrm{H} \beta$ to better than $15 \%$.

The uncertainty on line measurement depends not only on the strength of the line but also on the continuum and sky background level. Any systematic error in the stellar continuum subtraction would also contribute to the error of the line flux. Here, we ignore the latter and only consider the Poisson noise contributed by the stellar continuum, the sky background flux, and the emission line itself. The median sky background in dark time at 
APO is 22.2 and $21.2 \mathrm{mag} \operatorname{arcsec}^{-2}$ in $g$ and $r$ bands, respectively. The outskirts $\left(r>0.5 R_{e}\right)$ of nearby galaxies are mostly fainter than this sky background. We place the science requirements in this sky-background-dominated regime. To make conservative estimates, we assume that the stellar continuum is as strong as the median sky background. In the very centers of galaxies, the stellar continuum could dominate over the sky background and yield lower $\mathrm{S} / \mathrm{N}$ for fixed line flux. This would only affect a small fraction $(\sim 20 \%$ in $g$ and $\sim 10 \%$ in $r$ ) of the areas we probe.

The uncertainty on line measurement depends on the details of the measurement method-whether it is summed over a wavelength window or fitted with a Gaussian would yield a different result. Here, for simplicity, we just require the peak amplitude to be measured to better than $15 \%$ for $\mathrm{H} \beta$ and $5 \%$ for $\mathrm{H} \alpha$.

In a $2 . " 5$-diameter aperture, assuming a fixed line width of $70 \mathrm{~km} \mathrm{~s}^{-1}$, which is roughly the instrumental dispersion, the above line fluxes correspond to a peak amplitude of 21.3 and $139 \mu \mathrm{Jy}$ for $\mathrm{H} \beta$ and $\mathrm{H} \alpha$ at $z=0.15$. Thus, the uncertainty from the background and the lines should be less than $3.15(\mathrm{H} \beta)$ and $6.95(\mathrm{H} \alpha) \mu \mathrm{Jy}$. The stellar continuum, assumed to be equal to the median sky background, has a flux density in $g$ and $r$ band of 23.6 and $59.2 \mu \mathrm{Jy}$ in a 2 ."5-diameter aperture. The sky-subtracted signal is the sum of the line amplitude and the stellar continuum, which is $44.9 \mu \mathrm{Jy}$ or $19.8 \mathrm{mag}$ for $\mathrm{H} \beta$, and $198 \mu \mathrm{Jy}$ or $18.2 \mathrm{mag}$ for $\mathrm{H} \alpha$. Summarizing, we require the $\mathrm{S} / \mathrm{N}$ to be greater than $(44.9 / 3.15=) 14.3$ for $g=19.8$ and $(198 / 6.95=) 28.5$ for $r=18.2$ in a 2 ".5-diameter aperture.

2. In order to measure the gas-phase metallicity gradient to better than 0.04 dex per $R_{e}$, we need to measure it over several $(>=4)$ elliptical annuli and with an accuracy better than 0.05 dex per annulus. We set the outermost annulus to be from $1 R_{e}$ to $1.5 R_{e}$ with thinner inner annuli as the $\mathrm{S} / \mathrm{N}$ is higher there. To reach this precision, using the R23 method (Kewley \& Dopita 2002) as an example, we find that a peak-amplitude-to-noise ratio of at least 7 is needed on [O II], $\mathrm{H} \beta$, and [O III]. This translates to a continuum $\mathrm{S} / \mathrm{N}$ greater than 10 per pixel near $\mathrm{H} \beta$. This requirement is on stacked spectra for an elliptical annulus.

3. For star-forming galaxies and newly quenched systems (younger than $1 \mathrm{Gyr}$ ), to measure the mean stellar age to better than 0.1 dex using the $4000 \AA$ break and Balmer indices requires a median $\mathrm{S} / \mathrm{N}$ of greater than 10 per pixel in $r$ band. This estimate is based on Kauffmann et al. (2003).

4. For quiescent galaxies, in order to measure the stellar age, metallicity, and abundance gradient to better than $0.1 \mathrm{dex}$ per decade in $R_{e}$, we need to measure this over at least four elliptical annuli to a precision of better than $0.12 \mathrm{dex}$ in each annulus. Based on our prototype data obtained in 2013 January using prototype hardware (Bundy et al. 2015; Wilkinson et al. 2015) and an estimation of the accuracy in deriving stellar population properties from SDSS spectra in Johansson et al. (2012), we found that empirically, at a median stacked $\mathrm{S} / \mathrm{N}$ of greater than 33 per pixel in the $r$-band continuum, we could measure stellar age to a precision of \pm 0.12 dex, metallicity to a precision of $\pm 0.1 \mathrm{dex}$, and abundance to a precision of \pm 0.1 dex. This is using the method of absorption-line indices in the Lick/IDS system. If we use full spectrum fitting, simulations show that we can determine age and metallicity to a precision better than 0.1 dex or better with even lower S/N (C. Conroy 2016, private communication). We went with the more conservative estimate in designing our survey.

5. The $\lambda_{R}$ measurement depends on the flux profile of the galaxy, the velocity field, and the velocity dispersion $(\sigma)$ field. According to Emsellem et al. (2007),

$$
\lambda_{R}=\frac{\sum_{i=1}^{i=N_{p}} F_{i} R_{i}\left|V_{i}\right|}{\sum_{i=1}^{i=N_{p}} F_{i} R_{i} \sqrt{V_{i}^{2}+\sigma_{i}^{2}}},
$$

where $F_{i}, R_{i}, V_{i}$, and $\sigma_{i}$ are the flux, center distance, velocity, and velocity dispersion in the $i$ th spatial bin. In order to make a simple estimate, we assume a quiescent galaxy with uniform $\sigma$ throughout and a flat rotation curve that sharply decreases to zero in the center. Basically, we are assuming that every spatial bin has the same $\sigma$ and $|V|$. In this case, $\sum_{i=1}^{i=N_{p}} F_{i} R_{i}$ can be canceled out in the above equation, and it becomes easier to tie the uncertainty on $\lambda_{R}$ to the uncertainties on $V$ and $\sigma$. The relation is

$$
\Delta_{\lambda}=\lambda\left(1-\lambda^{2}\right) \sqrt{\left(\frac{\Delta_{V}}{V}\right)^{2}+\left(\frac{\Delta_{\sigma}}{\sigma}\right)^{2}} .
$$

For a slow rotator with $\lambda_{R} \sim 0.1$ and $\sigma=100 \mathrm{~km} \mathrm{~s}^{-1}$, to obtain a precision of $\Delta \lambda=0.05$, we need an $\mathrm{S} / \mathrm{N}$ per bin of at least 17 per pixel in $r$ band, according to the velocity and dispersion uncertainty achieved in the prototype data. The galaxy also needs to be reasonably well resolved in order to measure the $V$ and $\sigma$ maps. According to experience, we require approximately 20 independent spatial bins in order to have a reliable measurement. Therefore, the final technical requirement is at least 20 spatial bins with $\mathrm{S} / \mathrm{N}$ per pixel of at least 17 . This requirement can be reached for the majority of the $1.5 R_{e}$ sample.

6. Enclosed masses will be derived via dynamical modeling (Cappellari 2008). The enclosed mass inferred by the models scales as predicted by the virial theorem $M \propto V_{\mathrm{rms}}^{2}=V^{2}+\sigma^{2}$. This implies that the fractional mass error is twice the fractional $V_{\text {rms }}$ error. In addition to this random error, there is typically a contribution from various modeling systematic effects of about $6 \%$ (e.g., Figure 9 of Cappellari et al. 2013). Assuming that the random and systematic percent errors add quadratically, with $\mathrm{N}$ bins in which we measure $V_{\text {rms }}$, the mass error scales with $2 / \sqrt{N}$ of the error on $V_{\text {rms. In order to }}$ measure the mass to $10 \%$ with four bins at $1.5 R_{e}$, we need to measure $V_{\mathrm{rms}}$ to $11.3 \%$ :

$$
\left(\frac{\Delta V_{\mathrm{rms}}}{V_{\mathrm{rms}}}\right)^{2}=\frac{V^{2}}{V_{\mathrm{rms}}^{4}} \Delta V^{2}+\frac{\sigma^{2}}{V_{\mathrm{rms}}^{4}} \Delta \sigma^{2} .
$$

From the test-run data, we found the following empirical relationship between $\mathrm{S} / \mathrm{N}$ and the uncertainties on $V$ and $\sigma$ :

$$
\begin{gathered}
\Delta V=75 /(\mathrm{S} / \mathrm{N})^{0.95} \\
\Delta \sigma=101 /(\mathrm{S} / \mathrm{N})^{0.96} .
\end{gathered}
$$


For simplicity, we assume that $\Delta V$ scales with $\mathrm{S} / \mathrm{N}$ in the same way as $\Delta \sigma$ to make a conservative estimate. For a system with $V_{\text {rms }}$ of $60 \mathrm{~km} \mathrm{~s}^{-1}$ (similar to our instrumental resolution), to measure it to $11.3 \%$, we need an $\mathrm{S} / \mathrm{N}$ per bin of 16 per pixel in one-quarter of an annulus.

7. The requirements on the dark matter fraction estimate are more difficult to derive. Ideally, we would determine the number of spatial elements needed and the precision required on the velocity and velocity dispersion in each element, which can then be turned into a spatial coverage requirement and an $\mathrm{S} / \mathrm{N}$ requirement. However, the real situation is more complex. The precision on the velocity and velocity dispersion measurement depends not only on the $\mathrm{S} / \mathrm{N}$ of the spectra but also on the strength of the absorption features (and hence the metallicity of the galaxy). The number of spatial elements (e.g., Voronoi bins with a threshold $\mathrm{S} / \mathrm{N}$, as described in Cappellari \& Copin 2003) one can construct depends on the surface brightness profile of the galaxy. Even if these factors are fixed, the precision of the dark matter fraction we can achieve also depends on the complexity of the kinematic structure of the galaxy.

To make progress, we assess whether we can meet our requirement using simulated galaxies assuming our baseline sample and observing strategy. Such simulations, in conjunction with the MaNGA prototype data, show that it is possible to recover dynamical estimates of the dark matter fraction within $1 R_{e}$ with anisotropic Jeans mass modeling (JAM; Cappellari 2008) to better than $10 \%$ in galaxies with the second moment of the velocity distribution function larger than $60 \mathrm{~km} \mathrm{~s}^{-1}$ and bundle sizes larger than 19 fibers (so that their velocity fields are also well resolved spatially).

Among the requirements above, the most stringent requirements are from the stellar population gradient measurement on quiescent galaxies and from the measurement on enclosed gravitating mass. Both of these come down to roughly the same requirement of a continuum $\mathrm{S} / \mathrm{N}$ greater than 33 per pixel in the $r$-band portion of the spectrum stacked across all fibers between $1 R_{e}$ and $1.5 R_{e}$ and across all exposures. We require the majority of our Primary sample galaxies to satisfy this requirement. It sets the final exposure time of our observations.

\subsection{Requirements on the Sample}

Besides our requirement on the depth of the observation, we also have requirements about the mass distribution of galaxies, spatial coverage, number density on the sky, and availability of environment information.

We have the following requirements on our sample selection.

(A) The sample selection needs to be simple and reproducible so that one can easily reproduce the statistical distribution of any galaxy property for a volume-limited sample down to a certain stellar mass.

(B) The sample needs to be representative at all stellar masses $\left(10^{9}<M_{*} / M_{\odot}<10^{12}\right)$ in order to have enough statistical power for studies of both high-mass and low-mass galaxies.

(C) The selection shall give more weight to galaxies with rare color-mass combinations in order to have enough statistical power to sample these rare galaxies or short stages of evolution.

(D) The spatial coverage in all target galaxies should be as uniform as possible in units of $R_{e}$. We desire radial coverage to $1.5 R_{e}$ along the major axis to allow a long baseline for gradient measurements and to cover most of the light. An elliptical aperture with semimajor axis of $1.5 R_{e}$ covers $75 \%$ of the light for an exponential disk and $60 \%$ for a de Vaucouleurs profile.

We also require coverage to $2.5 R_{e}$ for one-third of the sample to explore the very outer regions of galaxies. Large spatial coverage offers the opportunity to probe the gas metallicity gradient in the outskirts of disks that may connect with accretion of low metallicity or nearly pristine material from filaments, to probe the stellar population at large radius, to probe beyond the peak of the rotation curve in disks, and to measure the dark matter fraction where it dominates the potential. The benefit of large coverage also comes with substantial risk as the $\mathrm{S} / \mathrm{N}$ will be much lower and spatial resolution is sacrificed.

(E) The sample should maximize spatial resolution while satisfying the above requirements. Each galaxy needs to be resolved with at least three radial bins in order to measure gradients in stellar age, metallicity, etc.

(F) The total sample size should be about 10,000 , with an approximate 2-to-1 split between Primary $\left(1.5 R_{e}\right)$ and Secondary $\left(2.5 R_{e}\right)$ samples. The justification for the sample size was described in Bundy et al. (2015). Briefly, this size will allow us to study any galaxy property as a function of three independent variables (e.g., mass, SFR, and environment) with six bins in each variable and provide us with $\sim 50$ objects per bin that will yield a $5 \sigma$ detection in the median difference of that property between bins assuming that the intrinsic scatter of that property in each bin is of similar level to the median difference.

(G) The combined subsamples need to have a sky surface density high enough to enable efficient allocation of IFU bundles.

(H) The majority of the sample needs to have environment information available, and the allocation of bundles needs to be unbiased with respect to the environment.

(I) A significant fraction of the sample needs to overlap past and near-future $\mathrm{H}$ I observations. Attention shall also be paid to other ancillary data of interest, such as deep imaging in the optical and near-IR and accesibility for follow-up observation from unique facilities in the Southern Hemisphere (e.g., the Atacama Large Millimeter/ submillimeter Array [ALMA]).

Often the requirements described in the above sections conflict with each other, as the design of our survey must fit within the realities of a finite budget and limited observing time. First of all, our total available observing time is fixed to half of the dark time in $6 \mathrm{yr}$. Second, given the limited budget, we are restricted to the detector real estate provided by the Baryon Oscillation Spectroscopic Survey (BOSS) spectrographs. With fixed total observing time, getting deeper observations inherently conflicts with getting a larger sample size. With fixed number of fibers and detector real estate, obtaining higher spatial resolution conflicts with the desire of larger spatial coverage. Given the limited number of galaxies in the nearby universe, higher spatial resolution also conflicts with 
the efficiency of observing them. These factors need to be balanced against each other.

In practice, given the requirements above and the boundary conditions, we jointly optimize the fiber bundle size distribution and the sample selection (D. Wake et al. 2016, in preparation). With the $\mathrm{S} / \mathrm{N}$ requirements set by the science requirements, we were just able to achieve a sample of about 10,000 galaxies in $6 \mathrm{yr}$ at APO.

\subsection{Requirements on Data Quality}

Here we describe several requirements we impose on the quality of raw data.

\subsubsection{Flux Calibration}

As described above, we need to measure SFR and gas-phase metallicity to a certain precision. These measurements are based on emission-line strengths and line ratios. Flux calibration uncertainty will contribute to the total uncertainty in these measurements. In Yan et al. (2016), we have done detailed derivations to flow down the science requirements to the requirement on flux calibration. Here we briefly summarize. Our requirement on flux calibration is that it does not dominate the uncertainty in the SFR calibration. Specifically, we require the contribution to SFR error from flux calibration alone to be better than 0.05 dex. This results in a requirement of $3.7 \%$ relative calibration error between $\mathrm{H} \alpha$ and $\mathrm{H} \beta$ and an $8.1 \%$ absolute calibration error on $\mathrm{H} \alpha$. From the gas-phase metallicity requirement, in order to measure $\mathrm{O} / \mathrm{H}$ to better than $0.04 \mathrm{dex}$, using $[\mathrm{N} \mathrm{II}] /[\mathrm{O} \mathrm{II}]$ as the metallicity calibrator, we found that the relative calibration between these two lines needs to be better than $7 \%$.

\subsubsection{Quality of the Reconstructed PSF}

MaNGA is both a spectroscopy survey and an imaging survey as we are effectively obtaining an image at every wavelength. Therefore, besides requirements on the depth of the observation, we also need to set requirements on the image quality. Because variations in the PSF could change the enclosed flux within a fixed aperture, wavelength-dependent PSF variation (e.g., caused by differential atmosphere refraction [DAR]) would lead to errors in flux calibration. Our requirement on the relative calibration between [N II] and [O II] to be better than $7 \%$ therefore translates to requirements on the effective PSF and the uniformity of observing depth. As derived by Law et al. (2015), we have the following requirements, for the entire wavelength range covered by the BOSS spectrograph:

(A) The FWHM of the reconstructed PSF in the final data cube cannot vary by more than $20 \%$ across a bundle.

(B) The reconstructed PSF must have an axis ratio $(b / a)$ greater than 0.85 .

(C) The effective exposure time cannot vary by more than $15 \%$ across a bundle.

These requirements place constraints not only on how we conduct the observations but also on how we process the data to recontruct the image at each wavelength to a regular grid. The choices we make on the observing strategy and the image reconstruction algorithm have to be driven by the hardware setup and the seeing condition at the observatory. We will discuss the observing strategy below in Section 5.1. And we refer the readers to Law et al. (2016), who discussed the choices we made in the image reconstruction algorithm and compared our approach with the approaches adopted by the CALIFA survey as described by Sánchez et al. (2012) and by the SAMI survey as described by Sharp et al. (2015).

\subsubsection{Sky Subtraction}

Our spectra cover many interesting features in the near-IR. The Ca II triplet, $\mathrm{Na}$ I at $0.82 \mu \mathrm{m}$, and FeH Wing-Ford band are sensitive to the inital mass function (Cenarro et al. 2003; Conroy \& van Dokkum 2012a). The [S III] $\lambda \lambda 9069,9532$ emission lines, combined with [S II] and $\mathrm{H} \alpha$, make excellent estimators for ionization parameter and gas metallicity (Kewley et al. 2001). However, these wavelengths are also dominated by forests of sky emission lines. Thus, in order to take advantage of these features, we need to achieve high-quality sky subtraction. We require our sky subtraction to be Poisson limited, especially in the 8000-10000 ̊ range. The Poisson limit is required so that we can stack many spectra in the outskirts of a galaxy (or from many galaxies) to achieve the stated $\mathrm{S} / \mathrm{N}$ in earlier sections and to analyze weak features in the near-IR.

\section{HARDWARE}

Details of the instrument configuration are described by Drory et al. (2015). We summarize them briefly here. We use the BOSS spectrographs (Smee et al. 2013) on the Sloan Foundation Telescope (Gunn et al. 2006). We modified the fiber feed system used by BOSS so that we can use fiber bundles to achieve IFS. The detector real estate limits the number of fibers we could accomodate in each spectrograph. Bigger bundles result in fewer per field. As described in detail by D. Wake et al. (2016, in preparation), we jointly optimized the bundle sizes with our target selection and arrived at the 17 science IFU bundles per plate with five different sizes: 2 19fiber bundles, 4 37-fiber bundles, 4 61-fiber bundles, 2 91-fiber bundles, and 5 127-fiber bundles. All bundles are hexagonal in shape. Each fiber covers a $2^{\prime \prime}$ diameter aperture on the sky with a 2 ." 5 center-to-center spacing between fibers, yielding a $56 \%$ fill factor. The largest bundle has a long axis of $32 \operatorname{arcsec}$ and a short axis of 28 arcsec. We define the effective radius of the bundle to be the radius of a circle that has the same area as the bundle. Table 1 lists the salient features of the bundles. This bundle size distribution roughly matches the apparent size distribution of our targets. Each plate also contains 12 7-fiber mini-bundles and 92 single sky fibers. The sky fibers are always associated with the IFU bundles, as detailed in Table 1, and are always plugged within 14 arcminutes to the IFU bundles. In total, we have 1423 fibers that feed the two spectrographs, with 709 fibers in Spectrograph 1 and 714 fibers in Spectrograph 2.

We have built six sets of these fiber assemblies and installed them in each of six identical cartridges. A cartridge is a removable cylindrical box that holds the fiber assemblies and provides the interface between the focal plane of the telescope and the input to the spectrograph. Aluminum plates are mounted on one side of the cartridge; fibers and fiber bundles get plugged into holes on the plate; at the output of the cartridge, all fibers are aligned in one of two pseudo-slits that feed the two spectrographs. On the pseduo-slits, the 1423 fibers are organized into 44 groups mounted on "v-groove" blocks with small gaps in between. Depending on the bundle 
Table 1

Fiber Bundle Configuration

\begin{tabular}{|c|c|c|c|c|c|}
\hline $\begin{array}{l}\text { Bundle Size } \\
\text { (fibers) }\end{array}$ & Purpose & $\begin{array}{c}\text { Number of Bundles } \\
\text { per Cartridge }\end{array}$ & $\begin{array}{l}\text { Number of } \\
\text { Sky Fibers }\end{array}$ & $\begin{array}{l}\text { Long-axis Diameter } \\
(\operatorname{arcsec})\end{array}$ & $\begin{array}{l}\text { Effective Radius } \\
\text { (arcsec) }\end{array}$ \\
\hline 7 & Flux. Cal. & 12 & 1 & 7.0 & 5.45 \\
\hline 19 & Science & 2 & 2 & 12.0 & 7.73 \\
\hline 37 & Science & 4 & 2 & 17.0 & 7.73 \\
\hline 61 & Science & 4 & 4 & 22.0 & 10.00 \\
\hline 91 & Science & 2 & 6 & 27.0 & 12.27 \\
\hline 127 & Science & 5 & 8 & 32.0 & 14.54 \\
\hline
\end{tabular}

size, the fibers in each IFU bundle get assigned to one to four such v-groove block(s). Sky fibers associated with an IFU bundle are assigned to the same v-groove block(s) as the fibers in the bundle, and they are always positioned on the edge of the block so that there is minimal contamination of the sky spectra by galaxy light.

When the cartridge is mounted to the telescope, the plate is located on the focal plane of the telescope and the pseudo-slits are inserted into the spectrographs. The light from the fibers is collimated and then split by a dichroic beam splitter into a blue camera and a red camera. The blue camera covers from $\sim 3630$ to $\sim 6300 \AA$, and the red camera covers from $\sim 5900$ to $\sim 10300 \AA$. Finally, they are dispersed by a grism and recorded on CCDs of $4 \mathrm{~K}$ by $4 \mathrm{~K}$. Each $\mathrm{CCD}$ is read out by four amplifiers. The spectral resolution, which depends smoothly on wavelength, is around $R \sim 2000$ (see Section 7.4).

\section{SAMPLE DESIGN}

Full details of the sample design and tiling are given in D. Wake et al. (2016, in preparation). Here we give an overview and provide some insight on how the science requirements drive the sample design.

Given the science requirements, we desire a sample of nearby galaxies with predetermined redshifts, with which we can estimate the stellar mass or the absolute magnitude and the environment around each galaxy. The effective radius should also be reliably measured so that we can use it to define a sample with uniform spatial coverage. The NASA-Sloan Atlas (NSA) catalog ${ }^{40}$ provides an ideal basis from which to select our targets. It is based on SDSS imaging with improved background subtraction and deblending (Blanton et al. 2011), and is much more complete than SDSS photometry catalogs, especially for galaxies brighter than $r_{\mathrm{AB}}$ of 16 (our final sample ranges roughly between $r_{\mathrm{AB}}$ of 13 and 17). We used a newer version (v1_0_1) of the NSA catalog than what is available on the NSA Web site. This version will also be released as part of Data Release 13 (DR13) of SDSS.

From the NSA, we select a sample with a roughly flat stellar mass distribution. The derivation of stellar mass is model dependent. Therefore, instead of stellar mass, we use the absolute $i$-band magnitude $\left(M_{i}\right)$ as it has less dependency on model assumptions and is more easily reproducible. Thus, we build our sample to have a flat distribution in absolute $i$-band magnitude. The absolute magnitudes for the sample are derived using the software package kcorrect (v4_2) (Blanton \& Roweis 2007).

We would like to maximize spatial resolution while ensuring that the majority of the sample is covered to a certain radius.

\footnotetext{
${ }^{40}$ http://www.nsatlas.org
}

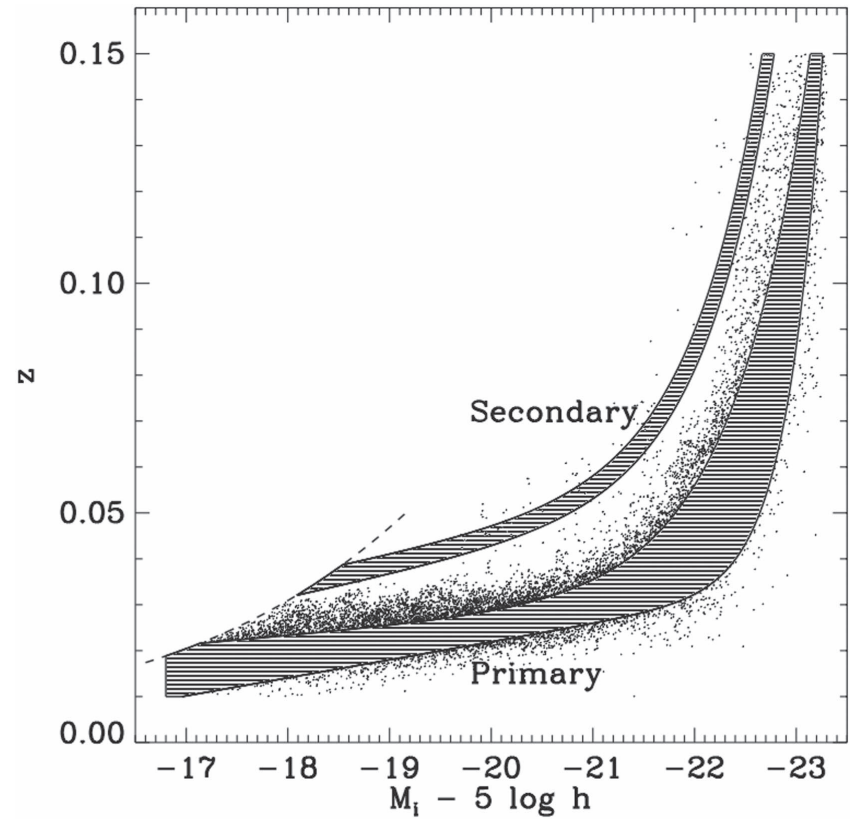

Figure 1. Luminosity-dependent redshift cuts we use to select the sample for MaNGA. The two shaded bands indicate the Primary sample (the lower band) and the Secondary sample (the upper band). The dashed curve indicates the completeness limit applied at faint magnitudes. Both the Primary and the Secondary samples are strictly volume limited at each $M_{i}$. The dots represent the color-enhanced supplement, which is built to oversample the underpopulated regions in color-magnitude space. See text and Wake et al. for more details.

The latter constraint means that we need to set a minimum redshift so that the angular size of galaxies can fit within our largest bundles. Because the sizes of galaxies increase with stellar mass or luminosity, the minimum redshift has to increase with brightening $M_{i}$. The maximum redshift is then set accordingly so that we have roughly the same number of galaxies in each absolute magnitude bin. The number per $M_{i}$ bin is set by the appropriate total number density of targets on the sky to ensure high completeness and high efficiency in targeting. In order to not bias the intrinsic sampling of galaxies, we conduct a volume-limited selection for each absolute magnitude. Our final luminosity-dependent redshift cuts are shown in Figure 1 and will be provided by D. Wake et al. (2016, in preparation).

MaNGA's main galaxy sample is composed of three components: Primary sample, Secondary sample, and the Color-Enhanced supplement. Both the Primary sample and the Color-Enhanced supplment aim to cover $1.5 R_{e}$; the Secondary sample is designed to reach $2.5 R_{e}$. To balance the potential science opportunity provided by the Secondary 


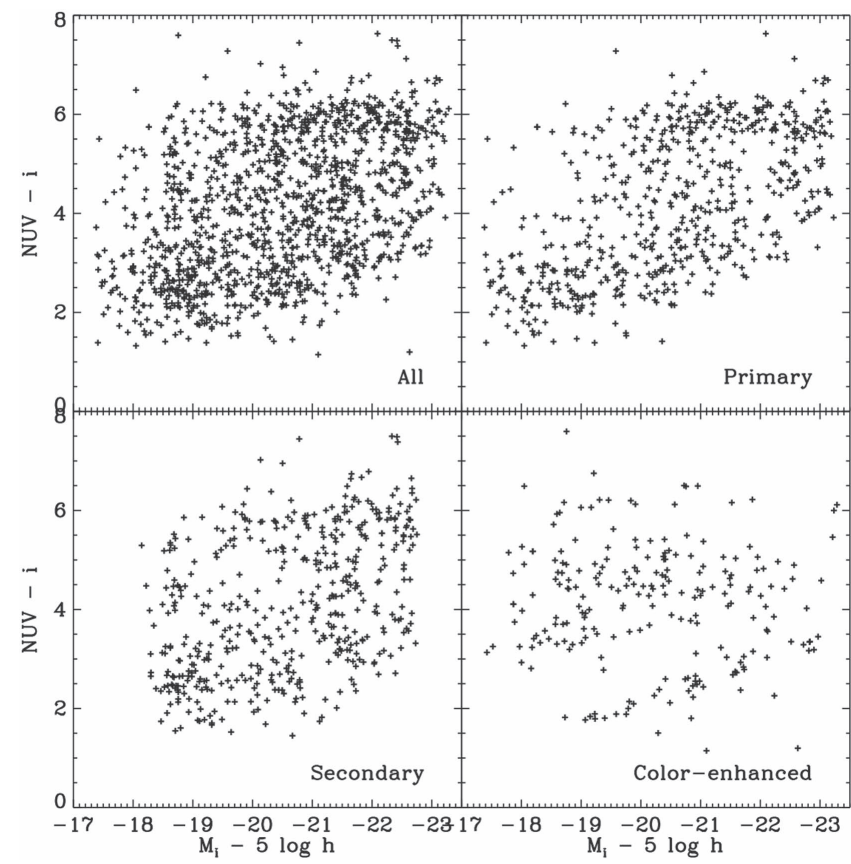

Figure 2. Color-magnitude distribution of galaxies observed in our first year of observations, which will be released in Data Release 13. Note that the color and magnitudes here are $k$-corrected to rest-frame and absolute magnitude, but are not corrected for internal extinction. Upper left panel: all targets observed; upper right panel: Primay sample; lower left panel: Secondary sample; bottom right panel: Color-enhanced sample.

sample and the risk associated with it, we decided to allocate one-third of the bundle resources to the Secondary sample. The other two-thirds will be spent on the Primary and ColorEnhanced samples. As they have the same spatial coverage goal, the Primary sample and the Color-Enhanced supplement are collectively termed the "Primary+" sample.

The Primary sample is a volume-limited sample at each absolute $i$-band magnitude, as is the Secondary sample. The Color-Enhanced supplment is designed to supplement the Primary sample by providing denser coverage in parts of the color-magnitude diagram with fewer galaxies. For example, green valley galaxies, massive blue galaxies, or the least massive red galaxies are rare in a volume-limited flat stellarmass sample. The mechanism to sample these rarer galaxies is to expand the redshift limits specifically for the relevant colormagnitude bins. The total number of the Color-Enhanced supplment is set to be one-third of the Primary sample. For details of how the supplment is defined, see D. Wake et al. (2016, in preparation).

In Figure 2, we show the color-magnitude distribution of galaxies observed in our first year of observations. The color and magnitudes here are $k$-corrected to rest-frame color and absolute magnitudes. However, there is no correction for internal extinction. Consequently, there are trends in the colormagnitude distribution that correlate with inclination, particularly for blue galaxies. This effect could be significant for the Color-Enhanced supplement as it selects preferentially face-on systems at blue colors and edge-on systems at red colors.

We would like to warn the users of the MaNGA sample that the sample is not a simple volume-limited sample with a single luminosity cut. Just taking the sample as a whole does not yield a representative sample of the galaxy population at any redshfit. For almost all science topics, one needs to carefully weight each galaxy by the inverse of its selection probability to reconstruct what a volume-limited sample would be like. In D. Wake et al. (2016, in preparation), we will provide the detailed prescription about how to reconstruct a volume-limited sample.

We allocate the targets to a large number of 7 square degree circular tiles that cover the entire SDSS DR7 footprint. The tiles are allowed to overlap to achieve roughly equal sampling completeness in different environments. Overall, we achieve a high allocation efficiency, with less than $2 \%$ of all fiber bundles unallocated. The tiles that do not have enough target galaxies were eventually filled with ancillary targets or galaxies outside our selection cuts. In total, there are about 1800 tiles, and we expect to only observe about 575-600 of them, given the limited observing time. The choice of which tiles to observe will be discussed below in Section 5.3.1.

There are a large number of ancillary science programs that could be done with the unique capability of the MaNGA instrument. In summer 2014, we had a call for ancillary proposals within the SDSS-IV collaboration. We received a large number of requests. Some of these could be used as fillers on plates with available bundles; others target rare objects that would not be observed unless we replace main sample targets with them. Given the large demand, we decided to dedicate 5\% of fiber bundles, in addition to the unallocated bundles, to ancillary target observations. In the end, $95.7 \%$ of all bundles are assigned to the main sample targets (45.1\% Primary, $15.0 \%$ Color-Enhanced, and $35.7 \%$ Secondary), and $5.1 \%$ are assigned to ancillary program targets. There is a $2.3 \%$ overlap between these two categories: some high-value ancillary targets also belong to our main sample. After including the ancillary targets, the total allocation efficiency is $98.5 \%$. When designing plates, the $1.5 \%$ of unallocated bundles are used to repeat some already-observed targets or assigned to randomly selected filler galaxies. We also achieve relatively high completeness in targeting: $85 \%$ of Primary sample and $66 \%$ of Secondary sample galaxies within our redshift cuts are targeted.

Once targets are assigned tiles, we allocate the differentsized IFUs according to the spatial coverage goals of the galaxies in that tile. Although the IFU size distribution is designed to match the size distribution of our targets, the matching is not perfect for each tile. When they do not match, we try to assign larger bundles to galaxies with a larger intended coverage. Figure 3 shows the final spatial coverage distribution of galaxies observed in the first year (DR13). Here the spatial coverage is defined by the ratio of a bundle's effective radius to the major axis $R_{e}$ of the target galaxy. A total of $69 \%$ of the Primary+ sample is covered beyond $1.5 R_{e}$, and $66 \%$ of the Secondary sample is covered to larger than $2.5 R_{e}$.

\section{DESIGN AND EXECUTION OF THE OBSERVATIONS}

In this section, we describe the operation of the survey in detail. We will first summarize how we set our observing strategy and plate completeness thresholds, and then we will describe how we chose the fields to observe, how we design the plates, and finally how we conduct the observations.

\subsection{Observing Strategy}

We first describe our observing strategy as it sets basic requirements for the operation. 


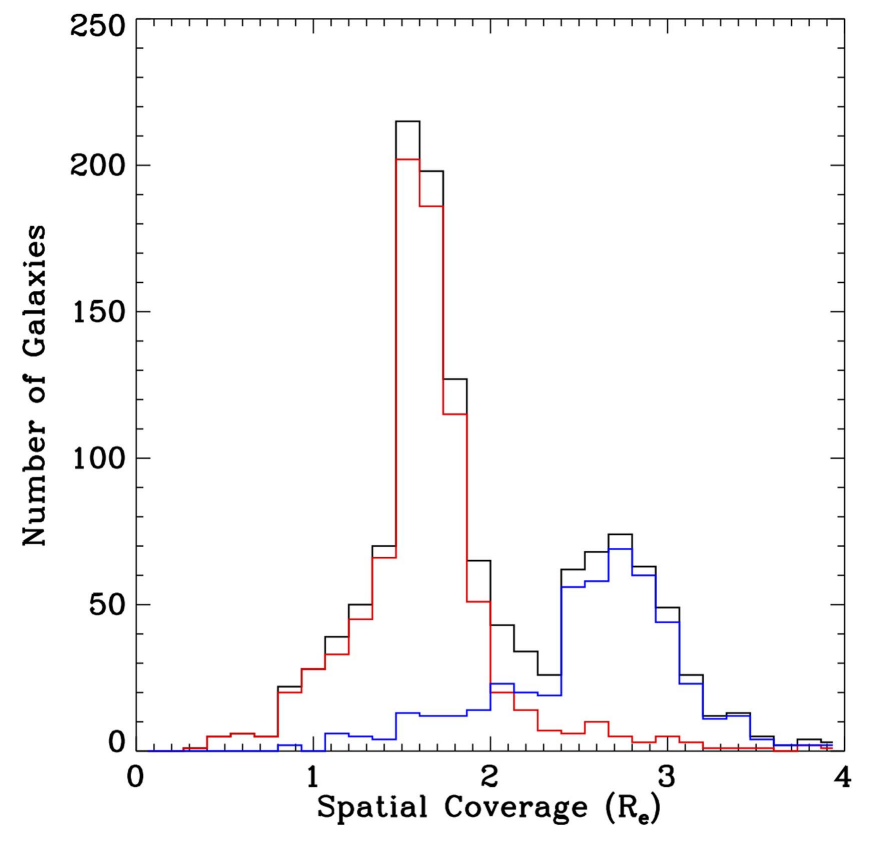

Figure 3. Spatial coverage distribution of galaxies observed in our first year of observations. The red histogram shows that for the Primary+ sample (Primary and Color-Enhanced). The blue histogram shows that for the Secondary sample. The black histogram is the total distribution.

Our fibers are $2^{\prime \prime}$ in size, while the typical seeing at Apache Point Observatory is 1".5, and the fiber-convolved point-spread function (FCPSF) is about 2".5, FWHM. The center-to-center spacing between fibers in our fiber bundle is $2 ! 5$. With a single pointing for exposures, we will not sufficiently sample the FCPSF. Thus, we need to conduct dithered observations. We employ a 3-point dither pattern that forms an equilateral triangle with 1!"44 on a side. This still does not Nyquistsample the FCPSF. In order to achieve Nyquist sampling, we would need to execute a 9-point dither, but that is not practical given the scheduling complexity. Therefore, we adopt the 3 -point dither as a compromise. The dither pattern is illustrated in Figure 6 of Law et al. (2015).

The MaNGA survey benefits from two unique capabilities provided by the Sloan Telescope and the BOSS spectrograph: the wide field of view and the wide wavelength coverage. However, these same features also bring complications. First, the large field of view results in a large variation in atmosphere refraction within the field, which compresses the field in the parallactic angle direction. Second, the wide wavelength coverage of the spectrograph results in a large chromatic DAR. Away from zenith, the extreme blue and extreme red wavelengths are both offset from the guiding wavelength.

Combining both effects, the exact dither pattern is a complicated function of the wavelength, the position of the field on the sky, and the time sequence of the dither pattern. Sometimes the two effects cancel, while at other times they add, making the dither more irregular. In order to maintain sampling uniformity, we have set constraints on where and how to execute the dither sequence. Law et al. (2015) did extensive simulations to study this effect. We came up with a metric termed the "uniformity statistic" $(\Omega)$, which is the maximum offset among all locations on a plate and across all wavelengths between the first and last exposures in a standard 3-point dither sequence due to both the uncorrected portion of the atmosphere refraction and the chromatic differential refraction. Generally speaking, $\Omega$ increases with the time separation between dither moves and with increasing airmass. Given our simulation, in order to satisfy the image quality requirements set in Section $2.3, \Omega$ must be less than 0 ". 4 for all wavelengths and all positions on a plate. With this requirement, we set the following guidelines for our observing strategy, which are justified in detail by Law et al. (2015).

1. Our exposures are set to 15 minutes each in order to maximize $\mathrm{S} / \mathrm{N}$ and not be overwhelmed by cosmic rays in the red detectors.

2. All exposures of a plate have to be taken within a predefined window centered on Meridian (referred to as "the visibility window"), with the length of the window set by the declination of the plate.

3. Each set of the three dithers must be taken within $1 \mathrm{hr}$ of each other in hour angle.

4. All exposures in a set must have individual seeing measures better than 2.!5 (FWHM), a min-to-max variation within the set less than $0 . " 8$, and a set-averaged seeing better than 2 ." 0 .

5. All exposures in a set need to have $(\mathrm{S} / \mathrm{N})^{2}$ within a factor of 2 from each other in all four cameras.

The visibility window is set by the declination of the field. The implemented visibility window length is different from the ideal case given by Law et al. (2015). The latter dictates very short windows for fields on the celestial equator or at decl. $<0$. This makes scheduling difficult at these declinations. Therefore, we relax it slightly for practical reasons. In the simulations presented by Law et al. (2015), we assumed that the time span of the three exposures (from the beginning of the first exposure to the end of the last exposure) is $1 \mathrm{hr}$, which allows for some moderate delays between exposure for unforseen situations. In reality, most of the time the exposures are executed consecutively without additional delays. Therefore, we assume a shorter window of only 48 minutes (three 15-minute exposures plus two readout/flushing overheads). This results in a much longer visibility window for low declinations.

The contraint that sets MaNGA's visiblity window limit is quite different from that of single-fiber spectroscopy, such as the BOSS and eBOSS surveys. For single-fiber spectroscopy, one is mostly concerned with the field differential refraction but not the chromatic differential refraction. And one can mitigate the AR effect by drilling the plate according to the planned observing time (Dawson et al. 2013). This helps the single-fiber surveys to cover time when the galactic plane is transiting. However, for MaNGA, the rotation of the chromatic DAR vector relative to our plate is what dominates the uniformity statistic at large hour angles and high airmass. The rotation cannot be addressed by the plate design. Therefore, the mitigation strategy used by BOSS/eBOSS to cover galactic plane transits is not applicable for MaNGA.

As a lesson for future fiber-based IFU surveys, if funding allows, an atmospheric dispersion corrector would be a valuable asset that would alleviate these problems.

\subsection{Plate Completeness Thresholds}

In this section, we discuss how the science requirements in Section 2 drive the plate completeness criteria in our observations.

As stated at the end of Section 2.1, our science requirements can be achieved if we can obtain an $\mathrm{S} / \mathrm{N}$ of 33 per pixel in the 
$r$-band continuum in the stacked spectra between $1 R_{e}$ and $1.5 R_{e}$. To achieve this in more than $75 \%$ of our target galaxies, we need a typical exposure time of $2.25 \mathrm{hr}$ (three sets of three 15-minute dithers) per plate in good conditions in sky areas with low galactic extinction. With this typical exposure time and depth, considering the good weather fraction at APO and all associated overhead, we will be able to observe $\sim 10,000$ galaxies in $6 \mathrm{yr}$ using half of the dark time. ${ }^{41}$

Doubling the $\mathrm{S} / \mathrm{N}$ would result in a sample size smaller by a factor of 4. A sample size of 2500 galaxies would be too small to meet our requirements. For example, if we want to study the dependence of a galaxy property on multiple variables (e.g., mass, color, environment), adopting several bins in each variable, we would quickly find too few galaxies per bin. A sample of 2500 also does not represent a significant advance from the sample currently available from surveys like CALIFA (667 galaxies) or SAMI (3400 galaxies). Going for 10,000 will enable much more additional discovery space, especially in terms of statistical signficance and rare galaxies. In addition, going for higher $\mathrm{S} / \mathrm{N}$ does not necessarily lead to smaller uncertainties in the measurements of physical properties. In the above discussion of science requirements, we have ignored the systematics associated with calibration and modeling, such as the uncertainty in SFR calibration, the systematics of the strong-line methods for measuring gas metallicity, the systematics associated with the stellar spectral libraries for stellar population modeling, and the uncertainty due to simplified star formation histories. At higher $\mathrm{S} / \mathrm{N}$, these systematical errors could dominate over the random noise and prevent us from taking advantage of the deeper observations. On the kinematics analyses, the uncertainties on the angular momentum, the enclosed gravitating mass, and dark matter fraction are also limited by spatial resolution $(\mathrm{Li}$ et al. 2016) or uncertainties in inclination determination.

In the other direction, we could consider shortening the exposure times to a minimum, which would be 45 minutes (one set of three dithers). This would triple the sample size with a decrease of $40 \%$ in $\mathrm{S} / \mathrm{N}$. With this option, the precision of many of our measurements would be comparable to the currently observed scatter in scaling relationships. This would prevent us from making progress in identifying the cause of the scatter.

Therefore, we consider our current choice of the $\mathrm{S} / \mathrm{N}$ threshold and the sample size of 10,000 the right balance to maximize the science return given the fixed amount of observing time.

Below, we describe several details in setting the completeness threshold and the lessons we learned in this process.

First, we cannot require every galaxy to reach equal depth as their sizes and surface brightnesses vary significantly. It is more practical to set a threshold on the total accumulated $(\mathrm{S} / \mathrm{N})^{2}$ for a fixed reference fiber magnitude (or the equivalent surface brightness), to ensure that the majority of them $(>75 \%)$ meet the science requirement.

Second, the reference fiber magnitude should be galactic extinction corrected. This would yield roughly equal depth regardless of foreground galactic extinction.

Third, although our science requirement is set on the $r$-band continuum, in reality, we do not track the $r$-band $\mathrm{S} / \mathrm{N}$ in real

\footnotetext{
41 Dark time is defined to be the time when the illumination fraction of the Moon is less than $35 \%$, or when the Moon is below the horizon and has an illumination fraction between $35 \%$ and $75 \%$.
}

time. This is because the BOSS spectrographs split the light into the blue and red cameras. The $r$ band is split by the dichroic beam splitter. Therefore, during observations, it is much easier to track the $g$ band and $i$ band, which are covered completely by the blue and red cameras, respectively. The correspondence between the $\mathrm{S} / \mathrm{N}$ in $g$ band (or $i$ band) and that in $r$ band (for fixed reference magnitudes) changes with the level of galactic extinction and the sky background. The actual $\mathrm{S} / \mathrm{N}$ thresholds have to be set through trial and error.

Our final completeness criteria for each plate are as follows: the total accumulated $(\mathrm{S} / \mathrm{N})^{2}$ among all exposures in complete sets must be above 20 pixel $^{-1}$ fiber $^{-1}$ in the $g$ band for an extinction-corrected fiber magnitude of $g=22$ and above 36 pixel $^{-1}$ fiber ${ }^{-1}$ in the $i$ band for an extinction-corrected fiber magnitude of $i=21$. We obtain as many complete dither sets until we meet these criteria.

We also learned a few lessons.

First, for projections of the survey speed, it is important to have an accurate prediction of how $\mathrm{S} / \mathrm{N}$ per exposure depends on seeing, airmass, and extinction in order to predict how many exposures are needed to reach the $\mathrm{S} / \mathrm{N}$ thresholds. A singlefiber survey and an IFS survey using the same telescope and instrument can have very different dependences of $\mathrm{S} / \mathrm{N}$ on these factors. At the beginning of our survey, we did not have enough data in hand to fully assess how our $\mathrm{S} / \mathrm{N}$ depends on these factors. Therefore, we adopted the $\mathrm{S} / \mathrm{N}$ relationships used by the SDSS-III/BOSS team, which led us to set too high an $\mathrm{S} / \mathrm{N}$ threshold. We thus exposed for too long initially on many plates and fell behind schedule. We corrected the problem in 2015 April.

The reason for this difference is that the $\mathrm{S} / \mathrm{N}$ obtained by single-fiber surveys such as BOSS is very sensitive to seeing, as they are targeting centers of galaxies where the surface brightness profile peaks. But for an IFU survey targeting outskirts of galaxies where the surface brightness is much flatter, the $\mathrm{S} / \mathrm{N}$ is almost independent of seeing. These different setups also yield different $\mathrm{S} / \mathrm{N}$ dependencies on airmass due to fiber alignment issues. Our actual $\mathrm{S} / \mathrm{N}$ dependence on airmass and extinction will be presented in Section 6.2 with Figures 9 and 10 and Equations (8) and (9).

Second, the requirement we set on the spatial sampling uniformity brings a cost to the observing efficiency. Because we dither and the three consecutive dithered exposures need to be obtained within $1 \mathrm{hr}$ of each other, sometimes the planned dither sequence can get interrupted by bad weather or bad seeing conditions, leading to a fraction of good exposures that cannot be combined into sets, which we refer to as "orphaned exposures." Sometimes, these orphaned exposures can be patched on subsequent nights with exposures with similar $\mathrm{S} / \mathrm{N}$ and seeing conditions into complete dither sets. Other times they are left behind and can account for $8 \%-10 \%$ of the total number of exposures. We do not include these exposures in the total $(\mathrm{S} / \mathrm{N})^{2}$ calculation or the reconstruction of the data cube. We trade a bit of observing efficiency for better data quality. These orphaned exposures could still be useful for certain science applications that require greater depth but less image quality.

\subsection{Field Planning}

\subsubsection{Overall Field Choices}

Given our observing strategy, the visibility window of each tile, the plate completeness thresholds, and the allocated time 
on the telescope, we can decide which tiles to observe. We consider the following factors in choosing the tiles. First, the tiles chosen need to cover sky regions available during a specific observing period. Second, we would like to overlap with several imaging surveys or surveys conducted in other wavebands to maximize the scientific return of the MaNGA data. Third, we would like to maximize $\mathrm{S} / \mathrm{N}$ obtained per hour. The second and third considerations do not necessarily agree with each other. We balance the two needs while maintaining the total number of galaxies and obtaining sufficient overlap with other surveys, with the following three considerations.

First, understanding the star formation, chemical evolution, and baryon cycles in galaxies requires knowledge about their gas content. Direct measurements of gas require H I observations at $21 \mathrm{~cm}$ and $\mathrm{CO}$ observations with submillimeter telescopes. We therefore choose to overlap significantly with the Arecibo Legacy Fast ALFA survey (Giovanelli et al. 2005), the planned Apertif Med-Deep fields (Verheijen et al. 2009), and low-declination regions accessible from ALMA.

Second, deep near-IR imaging can provide an independent measure of the stellar mass distribution in galaxies, which we can compare with the mass distribution measured from stellar population synthesis modeling of our spectra and that measured from kinematics, or combine to provide joint constraints. Therefore, we choose to overlap as much as possible with the United Kingdom Infrared Telescope Infrared Deep Sky Survey (UKIDSS) survey footprint (Lawrence et al. 2007).

Third, deeper and higher-resolution optical imaging than SDSS would help us identify internal structures in galaxies, such as small bars, spiral arms, etc., which will help us understand trends discovered in our data. At the same time, deep imaging could provide halo mass estimates from weak graviational lensing, which is independent of the other mass measurements we have. Therefore, we choose to overlap with the Hyper Suprime-Came (HSC) Survey deep imaging fields. ${ }^{42}$ In the Southern Galactic Cap, we have three stripes of SDSS legacy data. Here, we choose to prioritize Stripe 82 in order to take advantage of the many auxiliary data sets available there. These low-declination fields will provide a substantial sample for follow-up observation from ALMA and European Southern Observatory's Very Large Telescopes.

We developed a simulation tool to optimally choose the tiles for the available time, on a night-by-night basis. This simulation tool can be used to simulate the whole survey footprint or to decide which plate to drill for a particular drill run.

To simulate the whole survey footprint, we assume that $42.75 \%$ of the nights from now until summer 2020 are clear. This is the average good weather fraction after excluding open-dome time that is cloudy or has bad seeing, and after accounting for inefficiency caused by suboptimal conditions. ${ }^{43}$ Then for each night with scheduled observing time, the

\footnotetext{
42 http://www.naoj.org/Projects/HSC/index.html

43 Over the past $1.5 \mathrm{yr}$, we opened the enclosure in $50 \%$ of the time. Among the open-dome time, about $10 \%$ (5\% of the total) has seeing poorer than 2 ". 5 or has very low transparency that would not generate useful exposures. Among the $45 \%$ of the time with good seeing and not-so-bad transparency, good exposures are generated that we could potentially use in complete sets. Among all exposures that make up complete sets, a small fraction produces less than typical $\mathrm{S} / \mathrm{N}$, due to either suboptimal transparency or sky brightness. The last factor increases the total number of sets needed on our plates by $5 \%$. To account for these factors, in our survey simulation, we use a weather factor of $42.75 \%(50 \% \times 90 \% \times 95 \%=42.75 \%)$ and assume that all these times had typical clear conditions
}

simulation tool tries to find the best tile to be observed. We use an observing efficiency of $75 \%$ to account for overhead due to cartridge changes, acquistion, focusing, calibration, and readouts. We use the following logic in determining the best tile. Every tile has a visibility window that is determined by the declination of the plate. At the beginning of the observing period, we simulate all tiles that are visible. We predict the $S / N$ obtained in each exposure using the empirical relationship obtained from our first season of observations, which is a function of airmass and galactic extinction. We simulate observing all tiles until they are complete (accumulated S/N higher than the completeness threshold), until their visibility window expires, or until the observing time block finishes, whichever comes earlier. Among the plates that complete, we pick the one that requires the shortest time, with $\mathrm{S} / \mathrm{N}$ as the tiebreaker (higher $\mathrm{S} / \mathrm{N}$ is preferred). If none of the plates complete, we pick the plate with the highest completeness fraction. The simulation then continues with the time allocation. The plates that are partially complete are stored so that they can be used on a later night. The $\mathrm{S} / \mathrm{N}$ obtained in each exposure can be predicted according to empirical relations we obtained, which we describe in Section 6.2. The fields are chosen from the tiled list described in Section 4.

To prioritize tiles with auxillary data available, we separate all tiles into two different categories: those inside our favored regions and those outside our favored regions. We need to exhaust those inside before picking plates outside the favored regions. Figure 4 shows which tiles we will likely cover in the end. We will have significant overlap with several auxillary surveys in a number of places. Most of the tiles that do not overlap with any other auxiliary data are from already-observed fields. The fields on the edges of the NGC and SGC have to be observed becasue we need to make use of the allocated time when the galactic plane is transiting (around 5 and $19 \mathrm{hr}$ local sidereal time [LST]). Most of the already-observed fields were observed prior to the field optimization decisions.

\subsubsection{Monthly Plate Drilling Planning}

Spectroscopic observations using the Sloan Telescope need plugplates to be prepared in advance. Therefore, at least 5 weeks before the actual observations, we choose the fields to be observed and prepare the plates. Our actual planning for MaNGA usually follows a longer lead time to protect against potential problems in plate design, drilling, and shipping. During the planning phase, given the observing schedule over the next few months, we run a simulation to decide which tiles to observe. This uses essentially the same simulation tool described above, but prioritizes plates according to the following considerations. Plates that are already on the mountain are prioritized first, followed by those currently in the process of drilling or delivering, then followed by tiles we have not yet drilled. In this simulation, we also assume $100 \%$ good weather so that we will have enough plates in case of excellent weather.

\subsubsection{Daily Plugging Request}

At the mountain, after each night of observation, we have to decide which plates to observe on the next night so that they can be plugged during the day. This again requires the simulation tool mentioned above. In this case, we only consider plates that are on the mountain and have already been marked. 


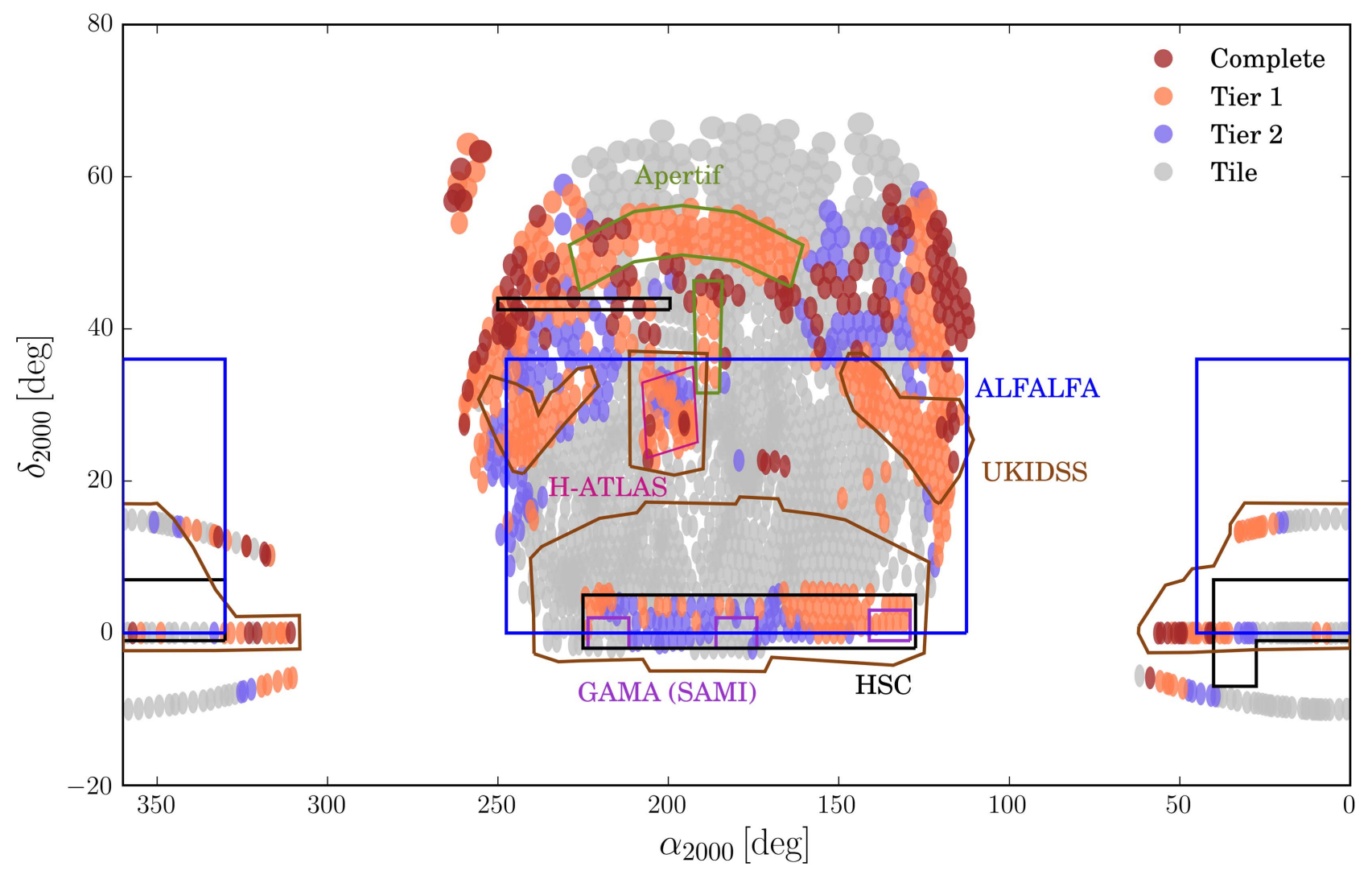

Figure 4. Existing and planned footprint of MaNGA on the sky. The gray circles illustrate our tiling of the SDSS Legacy survey footprint with $3^{\circ}$ diameter tiles. The red circles denote tiles we have already observed. The orange circles denote our planned footprint under the assumption that the average good weather fraction is $50 \%$ at all LSTs. These are our Tier 1 planned fields, and they have high likelihood to be observed by summer 2020. Blue circles denote the additional fields we will possibly observe (Tier 2) if the good weather fraction is $75 \%$ at all LSTs, which are less likely to be observed than Tier 1 . The historical good weather fraction at Apache Point Observatory is 50\%. For an up-to-date projection of the high-likelihood tile coordinates, see http://www.sdss.org/surveys/manga/forecast/.

We give highest priority to incomplete plates that are already plugged. Quite often, some partially completed plates may have individual dithers that do not form a complete set. When simulating the next night, if a plate with incomplete sets can have those sets patched, its priority will get significantly boosted. This minimizes the chance of leaving those incomplete sets behind. Because we have strict requirements on the seeing uniformity and $\mathrm{S} / \mathrm{N}$ uniformity among dithers in a set, when there is a partly cloudy night or a night with strongly variable seeing, our continuous dither sequence can be broken up into a number of incomplete sets. The patching of dither sets requires similar conditions (seeing, transparency, sky brightness) and similar hour angle of observations (see Section 5.1). We have built a Web interface (code-named "Petunia") to display incomplete set information for each plate and aid real-time plate choice decisions by the SDSS observers.

\subsection{Plate Design}

Our target selection process allocates targets from all samples, including ancillary programs, to specific tiles and assigns them bundles of a specific sizes. Before plates are drilled, the individual targets on those plates are visually inspected to ensure that their photometry measurements are reliable and the automatically determined centers are accurate. Through visual inspection, we identify those few percent of targets with problems. For galaxies with incorrect centers, we visually determine the correct centers; for galaxies with bad photometry, often due to a nearby saturated star, we reject them and replace them with other galaxies satisfying our selection cuts, including ancillary targets. If there are replacements, the bundle allocation algorithm is rerun so that the allocated bundle size for all galaxies on that plate is optimized. In the end, if we still have bundles left that are unallocated, we place those bundles with galaxies in the NSA catalog but outside the MaNGA selection cuts, with preferences given to those with the largest $R_{e}$.

The target selection only allocates the size of the bundle, but not the placement of the bundle. Since we have multiple bundles for each size, it does not matter which galaxy goes to which bundle as long as the bundle is of the right size. Therefore, among all galaxies assigned to a given sized bundle, we assign them to physical bundles according to their closeness to each bundle's anchoring point in the cartridge to minimize the chance of having a bundle's fiber cable stretched across the plate.

\subsubsection{Selection of Standard Stars}

For flux calibration, we target 12 standard stars on each plate simultaneously with the galaxy targets. These serve as standard stars. We select those with colors similar to the late F-type main-sequence stars. F stars are chosen here because they are relatively bright and very common in the Milky Way and have relatively smooth spectra. Hotter main-sequence stars would have even smoother spectra, but they are too rare at high galactic latitude. White dwarfs are also good standards, but they are too faint to be found in large numbers within the 
appropriate magnitude range. $\mathrm{F}$ stars also have relatively flat spectral energy distribution in $f_{\nu}$, yielding comparable $\mathrm{S} / \mathrm{N}$ in the blue and red portions of the spectral coverage. The cuts we use to select the F stars are the following:

$$
\begin{aligned}
m_{\mathrm{dist}}= & {\left[((u-g)-0.82)^{2}+((g-r)-0.30)^{2}\right.} \\
& \left.+((r-i)-0.09)^{2}+((i-z)-0.02)^{2}\right]^{1 / 2} .
\end{aligned}
$$

We select those stars with an observed magnitude $\left(\right.$ PSFMAG $^{44}$ ) range between 14.5 and 17.2 in the $g$ band. If we cannot find enough stars for a plate, we raise the faint limit to 17.7 , or 18.2 if necessary. For late $\mathrm{F}$ stars, this magnitude range ensures that they are beyond most of the galactic dust for the galactic latitude we observe $(|b|>19)$. Thus, we can safely use the dust map of Schlegel et al. (1998) to extinction-correct the colors when selecting them and apply the extinction correction to the model spectra when deriving the calibration vector.

We pick standard stars that are widely spread across each plate so that we sample a range of different airmasses. We allocate each IFU bundle to the standard star nearest its anchor block for ease of plugging.

\subsubsection{Selection of Sky Positions}

As described in Drory et al. (2015), we designed the sky fibers to be located near each fiber bundle to ensure that the sky subtraction is both close to the target on the sky and on the CCD. Different-sized bundles have different numbers of sky fibers. The 19-, 37-, 61-, 91-, and 127-fiber bundles have two, two, four, six, and eight sky fibers, respectively. For the minibundles assigned to the standard star bundles, each has one sky fiber associated with it. The sky fibers are physically connected with their respective IFU bundle, and they split off $280 \mathrm{~mm}$ above the ferrule. They have a roaming radius of 14 arcminutes around their associated IFU target. The sky positions are picked from a catalog of sky locations that contain no detections in the SDSS imaging survey. This is produced by the photometric pipeline as described by Stoughton et al. (2002).

After the plates are designed, the design files are sent to the drill shop at the University of Washington. Plates are drilled, measured, and then shipped to APO.

\subsection{Observing Preparation and Procedure}

At the mountain, when plates are received, they are handmarked according to the design to facilitate plugging.

Different from SDSS-III/BOSS and SDSS-IV/eBOSS, the plugging of the MaNGA IFUs is deterministic. Each IFU has a designated hole. Therefore, each IFU hole is marked with its ferrule plugging ID (FRLPLUG), which runs from 20 to 37 for the science IFUs and from 51 to 62 for the standard star IFUs. We started from 20 to avoid the confusion with the 16 guide fibers, which are also numbered. The associated sky fiber holes are also identified in the marking, but do not require deterministic plugging.

The plates for a given night are mounted into different cartridges before the fibers are plugged into the plate. The other ends of the fibers go to one of two pseudo-slits on the side of the cartridge. When a cartridge gets mounted to the telescope, the plate is on the focal plane, while the two pseudo-slits are

\footnotetext{
44 The PSFMAG we use is the same as given by SDSS Data Release 12. See http://www.sdss.org/dr12/algorithms/magnitudes/\#mag_psf.
}

inserted into the two BOSS spectrographs, in which the light is collimated, split into blue and red channels, dispersed, refocused, and recorded by the CCDs in the blue and red cameras separately.

After the plate is plugged, they are mapped by shining a laser beam down each fiber on the pseduo-slit end and stepping the laser along the slit so that each fiber gets illuminated sequentially. On the plate side, we cover the plate with a diffusion screen and record a video of the fiber illumination sequence. The video is then analyzed to find the correspondence between the fibers and the holes in which they are plugged. Since MaNGA IFU bundles are deterministically plugged, this step is only used to confirm that the bundles are plugged correctly and are passing light, and to map the sky fibers that are plugged arbitrarily within their own group. The resolution of the video camera is not sufficiently high to distinguish individual fibers within a bundle. The mapping of fibers within a bundle is also deterministic. They were built according to a specific mapping configuration for each sized bundle (see Drory et al. 2015). We verified the mapping within bundles on the test stand at University of Wisconsin. The information about fiber mapping in each bundle is recorded in a central metadata repository used by the data reduction pipeline (DRP; Law et al. 2016).

When the plate is bolted down inside the cartridge, it is slightly bent to mimic the shape of the focal plane at $5300 \AA$. The curvature and height of the plate are measured using a profiling bar that indexes to eight known radial locations on the cartridge. The bar consists of five digital linear micrometers placed at five radial positions on the plate. In total, 40 measurements across each plate are taken and stored in the plate database. If the plate curvature does not meet a specified tolerance range, the central pin is adjusted until all numbers are within specifications.

During night-time operations, the on-site observers mount the pre-plugged cartridge onto the telescope. Before observations, the spectrographs need to be focused. The focus can be adjusted at three places: the position of the collimator, which is pushed by three pistons, and the red camera and the blue camera, which can be adjusted by turning their respective focus ring. The red camera focus ring is usually held fixed. The measurement of the best focus is done by taking two arc lamp exposures with half of the collimated beam blocked by a Hartmann screen. The Hartmann screen can block either the left or right half of the beam. Two exposures are taken, one with the left half blocked and the other with the right half blocked. If the spectrograph is out of focus, one will detect a shift in the line centroid between these two Hartmann images. The shift can then be used to compute the movement needed to achieve best focus, based on data obtained from a focus sweep.

This focusing step is done everytime a new cartridge is mounted, but in a slightly different way for afternoon preparation compared to night observations. During afternoon checkout, we use the shift to determine the necessary collimator movement to get the red camera into focus. Then we retake another set of Hartmann exposures to determine the best blue camera ring adjustment needed to get the blue camera into focus. The blue camera focus ring needs to be moved manually, which can take several minutes to finish. During the night observations, to reduce the overhead, only the collimator is moved. We compute the movement needed to balance the 

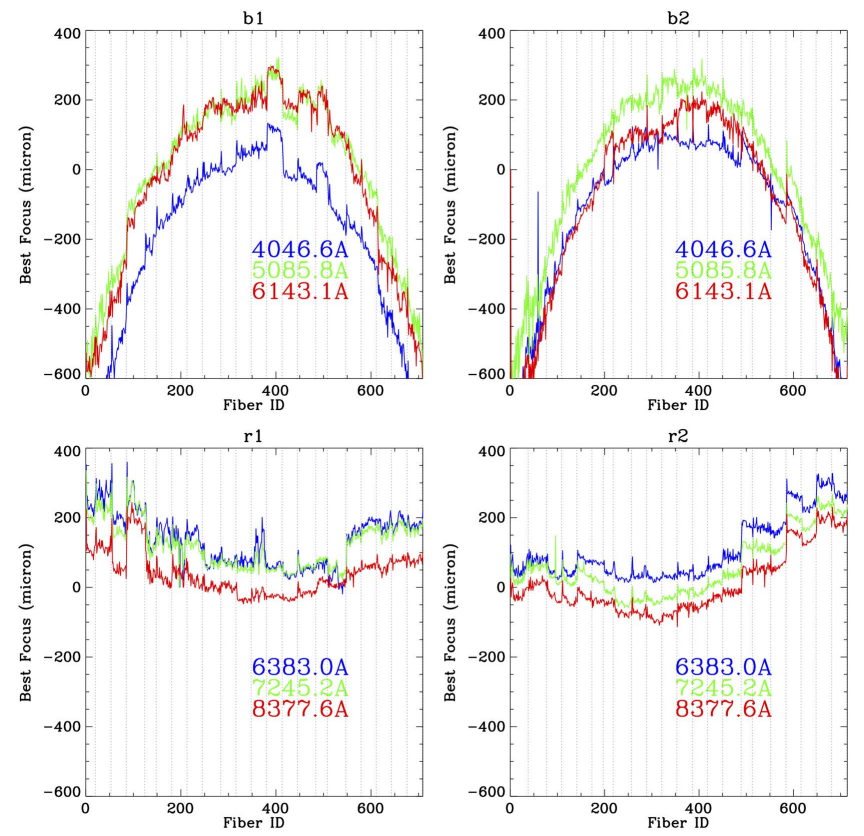

Figure 5. Best focus (expressed as the relative collimator position to produce the sharpest LSF) as a function of fiber ID for the blue and red cameras at a few different wavelengths. The thin dotted vertical lines mark the boundaries between v-groove blocks.

amount of defocus between the blue and the red cameras to reach an acceptable compromise.

The above description would have been accurate if the temperature does not change between afternoon checkout and night observations. In reality, the temperature change can significantly alter the relative focus between the blue and red cameras. Thus, during afternoon checkout, observers intentionally set the cameras out of focus in the right direction by an amount that is empirically determined, so that the relative focus between red and blue cameras stays within tolerance throughout the night.

After focusing, we take a $4 \mathrm{~s}$ arc lamp exposure and a $30 \mathrm{~s}$ (before MJD 57,325, 2015 October 29) or $25 \mathrm{~s}$ (on and after that date) flat field for calibration. We reduced the flat from 30 to $25 \mathrm{~s}$ integration because a region of the red camera in Spectrograph 1, which was replaced in 2014 July, becomes nonlinear in one quadrant above 35,000 ADUs. All $30 \mathrm{~s}$ flats taken before the switch are still in the linear regime. We reduced the exposure time to build a buffer zone for potential variations in flat lamp brightness. After calibration frames, we open the flat-field pedals of the telescope and acquire the field using first the two acquisition guide stars and then the other 14 guide stars. We then apply the first dither offset and start the exposure sequence. We take as many dithered exposures as necessary to complete a plate or until the end of its visibility window. We also take another set of arc and flat after the science exposures if we have stayed on the same field for more than an hour.

\subsubsection{Focus Optimization for MaNGA}

The focal plane of the spectrograph (relative to the surface of the CCD) at the position of the CCD is not flat. It is curved in both the dispersion direction and the spatial direction. Because the focus rings of the cameras are much more difficult to move than the collimator, we use the collimator moves to indirectly

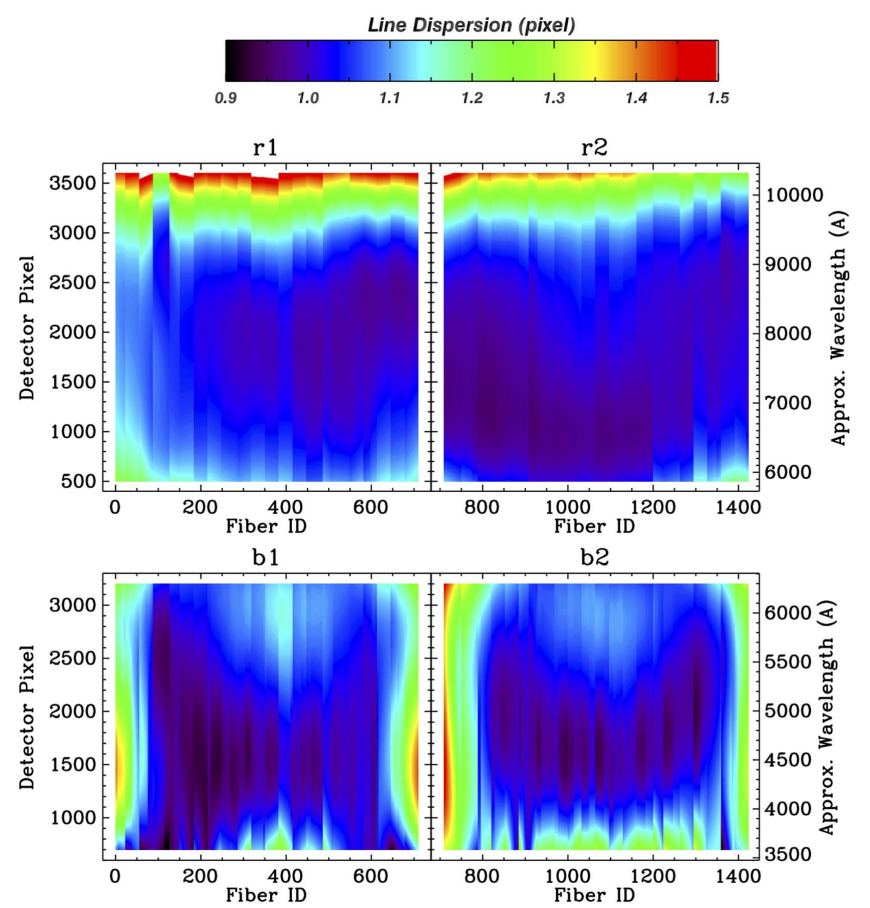

Figure 6. Maps of arc line dispersion on the detector for the four cameras. The $X$-axis is the spatial direction along the slit. The $Y$-axis is the dispersion direction.

probe the shape of the focal plane. We have conducted focus sweeps by moving the collimator from one side of the best focus to the other, taking a flat and an arc at each step. From these focus sweeps, we empirically determine the best focus for each location on the CCD, described by the position of the collimator that yields the sharpest arc line for that location. Figure 5 shows the best focus as a function of fiber ID for three wavelengths on each of the four cameras. We pick three arc lines in each camera located at the bottom, middle, and top of the CCD. From this figure, we see that the blue cameras have a much more curved focal plane than the red cameras. This causes larger resolution variations among fibers in the blue cameras. When the fibers near the center of the slit are in best focus, the edge fibers are significantly out of focus. In SDSSIII/BOSS, best focus is defined by the central fibers of the slit. This leads to relatively poor resolution in the edge fibers and steep resolution gradients within the fiber blocks at the edges. To mitigate this, we changed the compromise point to the position when fibers located at roughly one-fourth and threefourths the length of the slits are at their best focus. This is a better compromise as it minimizes the resolution variation among fibers. Figure 6 shows the result. The central fibers now have slightly worse resolution, but the dispersion in line width among fibers is significantly reduced while the same median line width is maintained.

The focal planes in the red cameras are much flatter. As shown in Figure 6, we have much less variation in resolution in the red cameras along the spatial direction. The dispersion of the red camera degrades significantly toward the red end of the spectrum, probably because these photons are absorbed at a significant depth within the thick chip. We adjusted the best focus for the red camera slightly so that the red end can be in a slightly better focus.

In the prototype observation conducted in 2013 January, we discovered that the red camera in Spectrograph 1 had 
significant coma at the red end of its wavelength coverage. We studied the history of the coma and found that it had been in existence since the summer shutdown of 2010. The cause turns out to be that the CCD was not placed at the correction position. This was corrected during the summer shutdown in 2014. There is still a small amount of coma left, but the quality returned to the same level as the beginning of SDSS-III. Our sky subtraction quality meets specification after this correction.

\subsection{Dithered Observations with Guider Offset}

MaNGA observation is dithered by executing guider offsets. The guider system uses 16 coherent imaging fiber bundles centered on 16 guide stars in the field. These imaging bundles are plugged in the same physical plate, but they are directed to a separate guider camera. The guider camera monitors the positions of the stars inside these 16 imaging bundles, taking a $15 \mathrm{~s}$ exposure every $30 \mathrm{~s}$. The images are analyzed to find the offset of the stars relative to their expected positions. The software solves for the optimal axes, rotation, and scale offsets to correct the pointing of the telescope. This keeps all the science fibers pointed at their respective targets. The resulting pointing stability of the telescope is 0 ! 12 , which is the rms offset reported by the guider software.

To dither, we simply modify the location of the expected guide star position. Rather than requiring all the stars to be located at the center of the guider fiber bundle, we require them to be located 0 ". 83 from the center in the direction of the dither. This results in slightly worse pointing stability. The reason is that the guider bundle's rotational orientation affects the expected guide star position with the bundle. The angle can be measured in the lab to better than $10^{\circ}$. But there is a \pm 3.5 uncertainty when guide fibers are plugged into the holes from mechanical tolerances. A poor knowledge of the actual angle can propagate to an error in the expected position of the guide star. Another error source is poor knowledge about the exact rotation center in the guider bundle. This is determined from the flat-field image of the guider output. In a flat-field image, each guider bundle shows a small circle. The center of the circle is set to be the rotational center. However, given the mechanical error of the centering of the guider bundle within its ferrule and the plug hole tolerance, the actual rotation center can be slightly offset from the center of the flat-field circle. Additionally, the position and scale of the guider CCD could potentially change with time and flexure relative to the guider bundle output block on the side of the cartridge. We do not know the level of impact of each of these factors. However, we can give an empirical assessment on our guiding accuracy from our data because we are doing IFS. Comparing our data with imaging reveals exactly how the galaxies are positioned relative to our fiber bundles. We present this comparison in Section 7.3.

\subsection{Quicklook Verification of Data Quality}

As mentioned above, we have a quick-reduction pipeline running at APO called "DOS." As soon as each exposure finishes reading out, DOS does a quick-and-dirty reduction of the data. It outputs the $\mathrm{S} / \mathrm{N}$ obtained for each fiber in the blue and red cameras. It then plots them as a function of expected fiber magnitudes based on the SDSS imaging. It does a linear fit with fixed slopes between the $(\mathrm{S} / \mathrm{N})^{2}$ and the fiber magnitudes for magnitudes between 20.5 and 22.5 in the
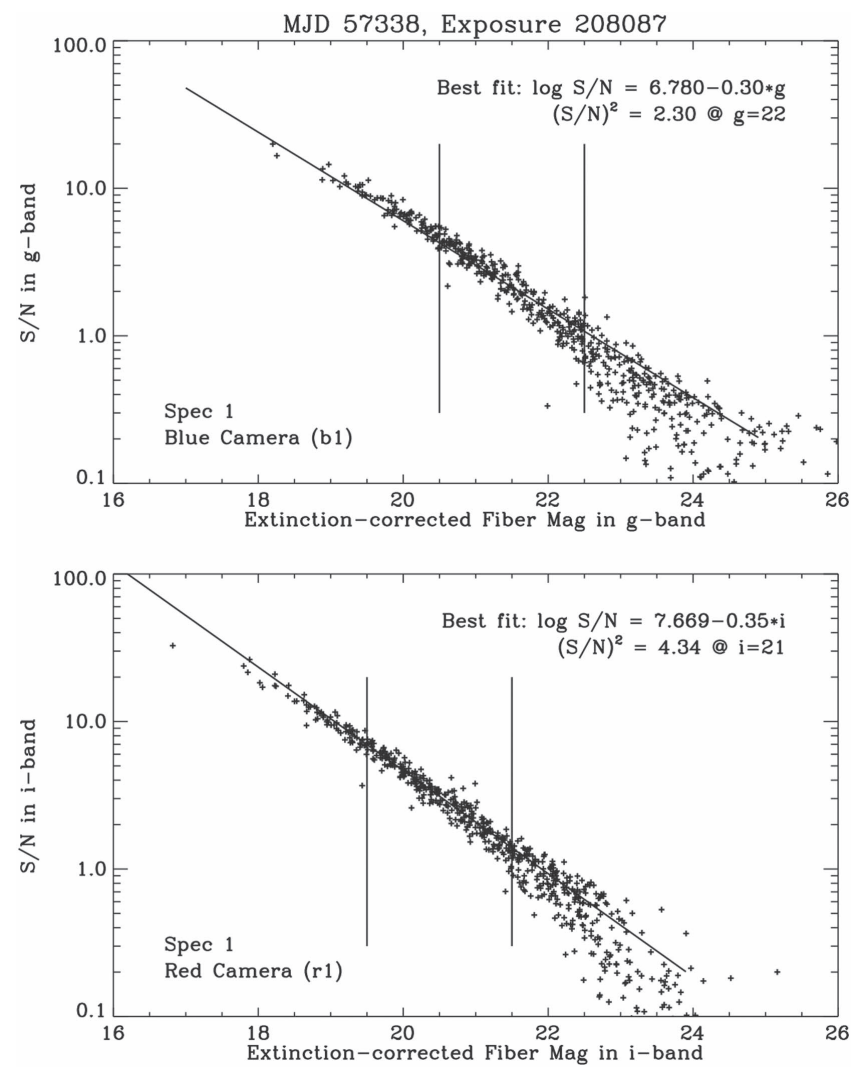

Figure 7. On-site quick-reduction results showing the $\mathrm{S} / \mathrm{N}$ for all fibers in Spectrograph 1 targeting galaxies as a function of the galactic-extinctioncorrected fiber magnitudes computed from SDSS images. The vertical lines indicate the range over which we fit the linear relationship with a fixed slope. The top panel is for the blue camera, for which the $\mathrm{S} / \mathrm{N}$ is measured in $g$ band and fit as a function of $g$ magnitude. The bottom panel is for the red camera, for which the evaluation is done in $i$ band.

$g$ band and between 19.5 and 21.5 in the $i$ band. It then outputs the best-fit $\mathrm{S} / \mathrm{N}$ for $g=22$ and $i=21$ as the reference $\mathrm{S} / \mathrm{N}$ for this exposure. As we have two spectrographs and each has two cameras, we have four $(\mathrm{S} / \mathrm{N})^{2}$ values output for each exposure. Figure 7 shows examples.

The plate completeness judgment is defined by the total accumulated $(\mathrm{S} / \mathrm{N})^{2}$ at the two reference magnitudes in the blue $(g=22)$ and red $(i=21)$, averaged between the two spectrographs. We require the average accumulated $(\mathrm{S} / \mathrm{N})^{2}$ in the blue to be greater than 20 pixel $^{-1}$ fiber $^{-1}$ and the $(\mathrm{S} / \mathrm{N})^{2}$ in the red to be greater than 36 pixel $^{-1}$ fiber $^{-1}$ for a plate to be considered complete.

\subsection{Data Reduction}

After each night of operations, the data are transfered from APO to the Science Archive Server at the University of Utah, where they are processed by the MaNGA DRP (Law et al. 2016). The DRP processes the raw data files and produces skysubtracted, flux-calibrated, co-added data cubes for each galaxy. There are two parts of the DRP: a $2 \mathrm{~d}$ stage and a $3 \mathrm{~d}$ stage. The $2 \mathrm{~d}$ stage processes the raw frames to produce one sky-subtracted and flux-calibrated 1d spectrum for each fiber in each exposure. The final spectra are sampled on a common wavelength grid. We provide two kinds of wavelength sampling: evenly spaced in logarithmic wavelength and evenly spaced in linear wavelength. The $3 d$ stage of the DRP 


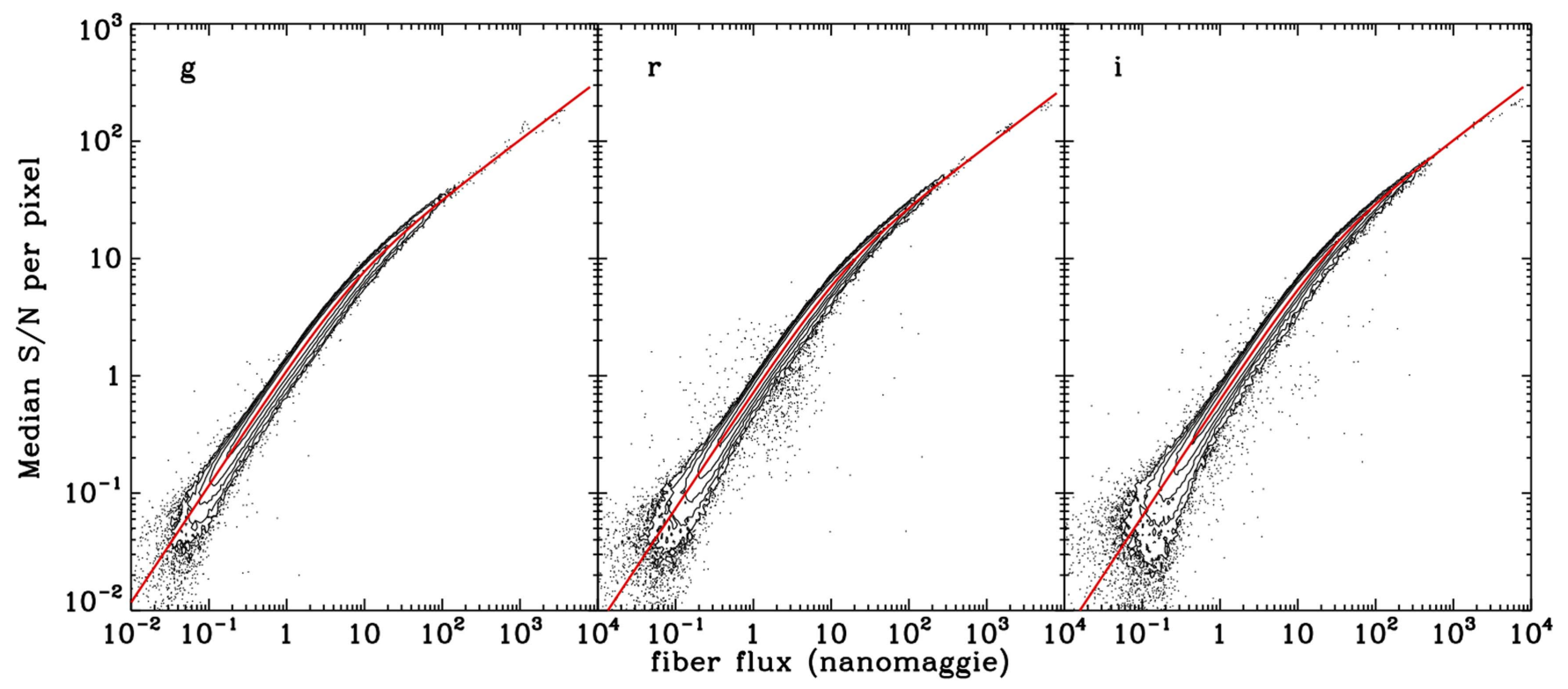

Figure 8. $\mathrm{S} / \mathrm{N}$ per pixel in $g, r$, and $i$ bands as a function of the synthetic fiber flux computed from the flux-calibrated spectra.

combines all the 1d spectra associated with each target from all exposures to produce the final data cube for that target, with astrometry adjustments made to each exposure by comparing the data against SDSS images. Besides the final data cube, the $3 \mathrm{~d}$ stage also makes available all the 1d spectra per fiber, per exposure associated with each target in a row-stacked form. This is referred to as the "row-stacked spectra" (RSS). The DRP is described in detail by Law et al. (2016).

\section{S/N ACCUMULATION SPEED AND SURVEY PROGRESS}

\subsection{S/N as a Function of Fiber Magnitude}

Here we present the $\mathrm{S} / \mathrm{N}$ obtained as a function of the fiber magnitude (or surface brightness). This differs from our original expectations based on archival data from the BOSS survey, as explained below.

For each 1d spectrum we obtain per exposure, we compute the median $\mathrm{S} / \mathrm{N}$ per pixel $\left(\Delta \log \lambda=10^{-4}\right)$ in each of the four optical bands $(g, r, i$, and $z$ ) that are completely covered by the spectra. The wavelength windows used for the median calculation are 4400-5500 $\AA$ for $g, 5601-6749 \AA$ for $r$, 6910-8500 $\AA$ for $i$, and 8284-9281 $\AA$ for $z$. We derive an empirical relation between the median $\mathrm{S} / \mathrm{N}$ per pixel and the flux-calibrated synthetic fiber magnitudes, with the latter calculated from flux-calibrated spectra. These are shown in Figure 8 . We fit these relationships with the following functional form:

$$
\mathrm{S} / \mathrm{N}=\frac{a F}{\sqrt{F+b}} .
$$

Here $F$ is the synthetic fiber flux in units of nanomaggies; ${ }^{45} a$ and $b$ are fitting parameters. We chose this form because it captures the ingredients of an actual $\mathrm{S} / \mathrm{N}$ estimation based on Poisson statistics and provides a very good match to the data, as shown in Figure 8. The resulting fitting parameters for the $g$, $r$, and $i$ bands are given in Table. 2. The relationships are much

\footnotetext{
$\overline{45}$ https://www.sdss3.org/dr8/algorithms/magnitudes.php
}

Table 2

Parameters in Equation (7) That Relate Our S/N with Flux in Fibers

\begin{tabular}{lcc}
\hline \hline Band & $a$ & $b$ \\
\hline$g$ & 3.41710 & 7.65072 \\
$r$ & 2.89589 & 12.9510 \\
$i$ & 3.23293 & 23.4808 \\
\hline
\end{tabular}

tighter than what we get when using fiber magnitudes computed from imaging as there are no added uncertainties from astrometry error, PSF mismatch, or photometry calibration. These formulae can be useful for $\mathrm{S} / \mathrm{N}$ predictions for future IFU observations with the SDSS Telescope and BOSS spectrographs.

This set of coefficients can be used to compute the typical $\mathrm{S} / \mathrm{N}$ we obtain in the data cube for a certain apparent surface brightness. First, convert surface brightness to $2^{\prime \prime}$ diameter fiber flux in units of nanomaggies. Then use Equation (7) and coefficients in Table 2 to get the $\mathrm{S} / \mathrm{N}$ per pixel per exposure. Finally, multiply by the square root of the number of exposures (typically 9 for sky regions with low galactic extinction) and the square root of the fiber filling facotr $(0.56)$. The result is the $\mathrm{S} / \mathrm{N}$ per pixel one would obtain in the final data cube in a $2^{\prime \prime}$ diameter aperture. The pixel size is $\Delta \lambda=10^{-4} \lambda \ln 10$. Typically, for an apparent $r$-band SB of $22.5 \mathrm{mag} \operatorname{arcsec}^{-2}$, the final $\mathrm{S} / \mathrm{N}$ in the data cube over a $2^{\prime \prime}$-diameter aperture is about 5.1 .

\subsection{Exposure S/N Dependence on Airmass and Galactic Extinction}

Given the $\mathrm{S} / \mathrm{N}$ estimates made by our DRP, we can empirically determine the dependence of our $\mathrm{S} / \mathrm{N}$ on airmass and extinction. Our $\mathrm{S} / \mathrm{N}$ depends weakly on airmass. There are several factors that degrade the $\mathrm{S} / \mathrm{N}$ when we go to higher airmass. First, there is more atmospheric extinction. Second, there is a brighter sky background. For single-fiber spectroscopy like BOSS/eBOSS, $\mathrm{S} / \mathrm{N}$ is also degraded at high airmass due to both larger field differential refraction and chromatic 

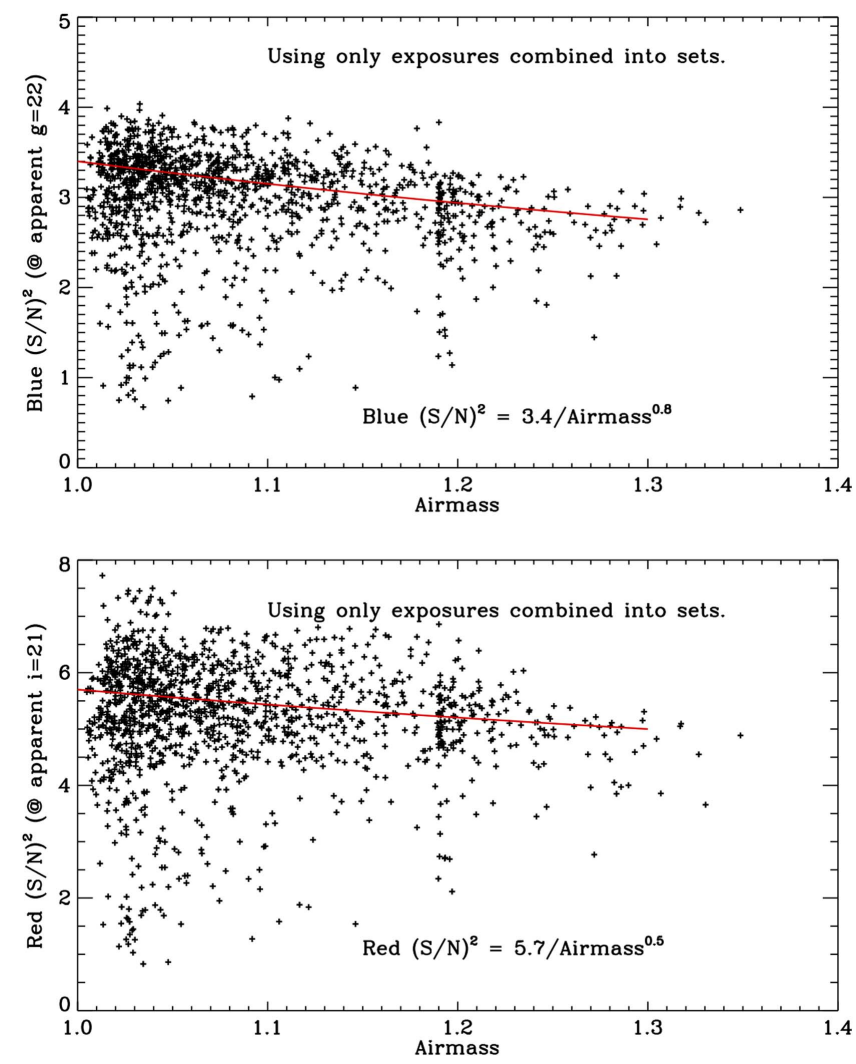

Figure 9. $(\mathrm{S} / \mathrm{N})^{2}$ per exposure at fixed apparent magnitude (before galactic extinction correction) as a function of airmass. The large scatter is due to variations in sky background and transparency. The reference magnitudes are $g=22$ in the blue and $i=21$ in the red. The lines represent an empirical relationship at typical good conditions. Only exposures that make complete sets are included, as the exposures with orphaned dithers tend to be biased to lower $\mathrm{S} / \mathrm{N}$.

field differential refraction. Because MaNGA is using fiber bundles to cover galaxies, DAR simply shifts flux from one fiber to the next and does not affect our $\mathrm{S} / \mathrm{N}$. Therefore, we have a weaker $\mathrm{S} / \mathrm{N}$ dependence on airmass than BOSS/ eBOSS. Figure 9 shows how our $\mathrm{S} / \mathrm{N}$ depends on airmass. Note that here we are using the $\mathrm{S} / \mathrm{N}$ at fixed apparent magnitude before correcting for galactic extinction, because we want to separate the dependence on airmass from the dependence on galactic extinction. This is derived from fitting the $\log \mathrm{S} / \mathrm{N}$ versus apparent magnitude using the same fixed slope relation as used in DOS, except that we do not apply galactic extinction.

The reason for the large scatter in Figure 9 is variations in transparency and sky brightness, with the Moon and clouds both contributing to the sky brightness variation.

We estimate our $\mathrm{S} / \mathrm{N}$ at galactic-extinction-corrected magnitudes. Therefore, our $\mathrm{S} / \mathrm{N}$ also depends on galactic extinction. Extinction simply shifts our apparent magnitude in the $\log \mathrm{S} / \mathrm{N}$ versus magnitude plots. Therefore, the effect of extinction should be directly related to the slope of the relation. In Figure 10, we show the ratio of the $(\mathrm{S} / \mathrm{N})^{2}$ derived from galactic-extinction-corrected magnitudes to those derived based on uncorrected magnitudes, and we plot them against the median galactic extinction of all target galaxies on a plate, for all exposures. The coefficients in the exponent should be twice the slope used in Figure 7 . The best-fit relation is slightly different. This is because for plates with significant extinction,
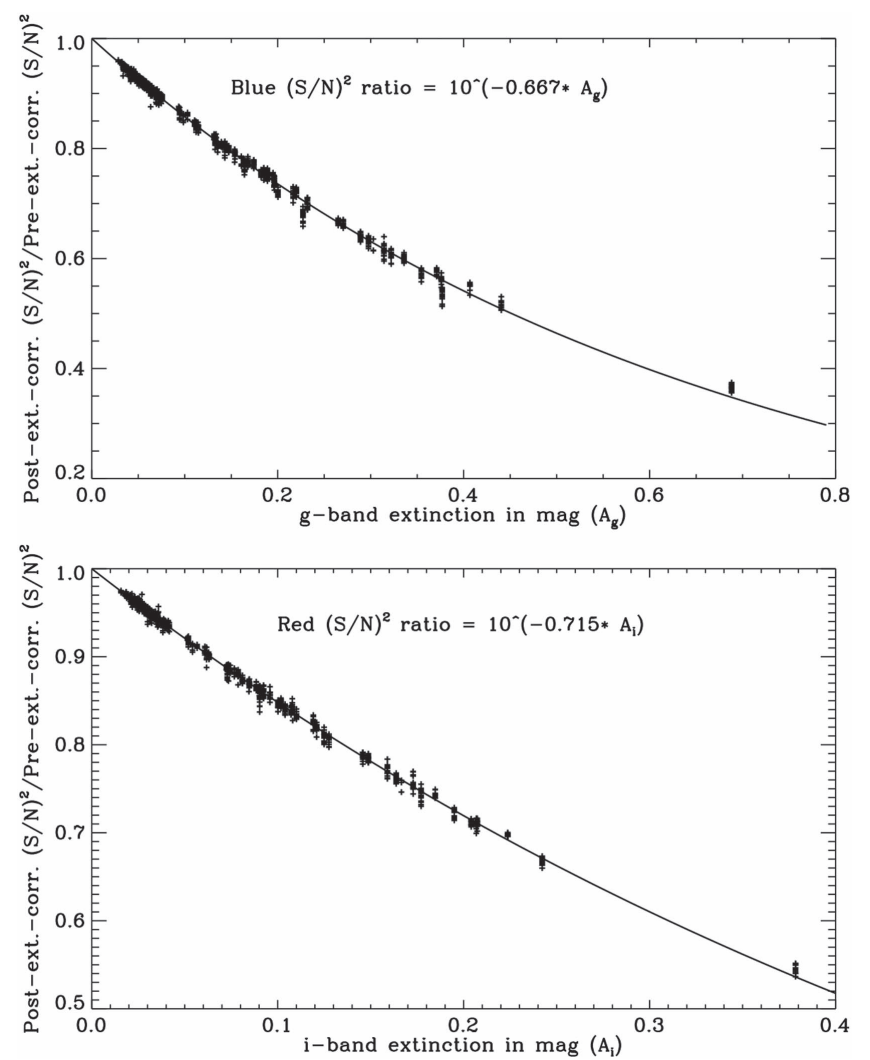

Figure 10. Impact of the galactic extinction correction on obtained $(\mathrm{S} / \mathrm{N})^{2}$ for the $g$ band (upper panel) and the $i$ band (lower panel). The ratio of the corrected vs. uncorrected $(\mathrm{S} / \mathrm{N})^{2}$ values is plotted against the strength of the correction in magnitudes. The lines represent empirical relationships that were designed to fit the high-extinction regime, where the corrections are most important.

the fixed reference magnitudes correspond to fainter apparent magnitudes where the slopes are slightly steeper. BOSS assumed $(\mathrm{S} / \mathrm{N})^{2}$ scales with $10^{-0.8 \mathrm{~A}}$, which is appropriate for the background-dominated regime. The reference magnitudes BOSS and MaNGA use are significant compared to the sky backgrounds, leading to a shallower slope.

Given the empirical dependence of $\mathrm{S} / \mathrm{N}$ on airmass and extinction, we arrived at the following equations for our observing speed:

$$
\begin{aligned}
& (\mathrm{S} / \mathrm{N})_{\text {Blue }}^{2}=\frac{3.4}{\text { Airmass }^{0.8}} \times 10^{-0.667 A_{g}} \\
& (\mathrm{~S} / \mathrm{N})_{\text {Red }}^{2}=\frac{5.7}{\text { Airmass }^{0.5}} \times 10^{-0.715 A_{i}}
\end{aligned}
$$

Here $A_{g}$ and $A_{i}$ are the galactic extinction in $g$ and $i$ bands, respectively.

One thing worth noting is that our $\mathrm{S} / \mathrm{N}$ has no dependence on seeing. Single-fiber spectroscopy targeting centers of galaxies, such as BOSS and eBOSS, has a very strong dependence on seeing, because the surface brightness profiles of galaxies peak at the center and are relatively shallow on the outskirts.

\subsection{S/N Prediction and Expected Survey Speed}

Given the above empirical relationship between $\mathrm{S} / \mathrm{N}$ and fiber magnitudes, we can estimate the stacked $\mathrm{S} / \mathrm{N}$ obtained in 


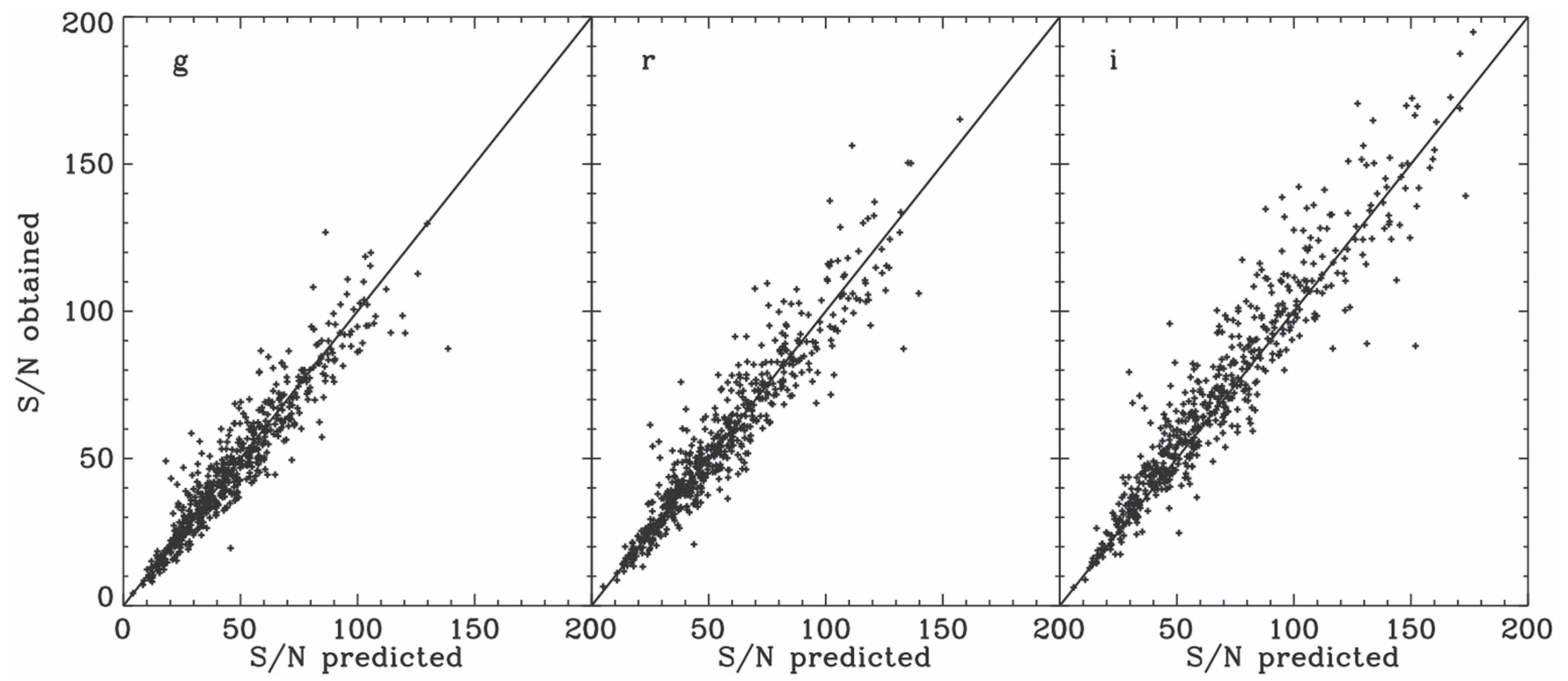

Figure 11. Measured $\mathrm{S} / \mathrm{N}$ compared to predicted $\mathrm{S} / \mathrm{N}$ based on photometry in the $g, r$, and $i$ bands, for an elliptical annulus between 1 and 1.5 effective radii of the galaxy or between two-thirds and 1 effective radius of the bundle, whichever is smaller. We display only galaxies that were observed in the first year with the updated completeness threshold. The solid line marks the $1: 1$ relation. The actual data have slightly higher $\mathrm{S} / \mathrm{N}$ than our predictions in $i$ band.

the outskirts of our target galaxies and check this against our science requirements.

We estimate the $\mathrm{S} / \mathrm{N}$ from surface photometry in an outer elliptical annulus. The annulus is set to have the axis ratio and the orientation given by the NSA catalog. The semimajor axis range of the annulus is set to between $1 R_{e}$ and $1.5 R_{e}$ or between two-thirds and 1 effective radius of the fiber bundle hexagon, whichever is smaller. For the smallest bundle we have, for a circular galaxy that has $1.5 R e$ greater than or equal to the effective radius of the bundle (5". 45), this corresponds to the outer ring of fibers.

Using the images provided by the NASA-Sloan Atlas, we convolve the images to the seeing of the observation before computing the $2^{\prime \prime}$ aperture photometry at the positions of all fibers in a bundle that is centered on the galaxy. We use the resulting fiber flux to estimate the $\mathrm{S} / \mathrm{N}$ obtained in the defined elliptical annulus. Many fibers lie across boundaries of the annulus, for which we only count the flux in proportion to the fraction of the fiber area that lies within the annulus.

We compared this $\mathrm{S} / \mathrm{N}$ prediction with the stacked RSS spectra of galaxies. We first multiply the flux from each fiber by the fraction of the fiber's area within the annulus, and then we add them together. The variance is propagated accordingly. We then compute the median $\mathrm{S} / \mathrm{N}$ in four wavelength windows corresponding to griz bands. Figure 11 shows the comparison between the predicted $\mathrm{S} / \mathrm{N}$ based on previous photometry and that from the stacked RSS spectra. The data are very consitent with our predictions in the $g$ and $r$ bands and show slightly better $\mathrm{S} / \mathrm{N}$ in the $i$ band.

\subsection{Final S/N Distribution and Projection}

The top panel of Figure 12 shows the actual $\mathrm{S} / \mathrm{N}$ distribution in stacked spectra in an elliptical annulus between $1 R_{e}$ and $1.5 R_{e}$ of the galaxy (or between two-thirds and 1 effective radius of the bundle, if the galaxy is not covered to $1.5 R_{e}$ ) for the Primary + sample. Our science requirement is to have an $\mathrm{S} / \mathrm{N}$ greater than 33 for more than $75 \%$ of the sample. In the first year of observation, $88 \%$ of the Primary+ sample
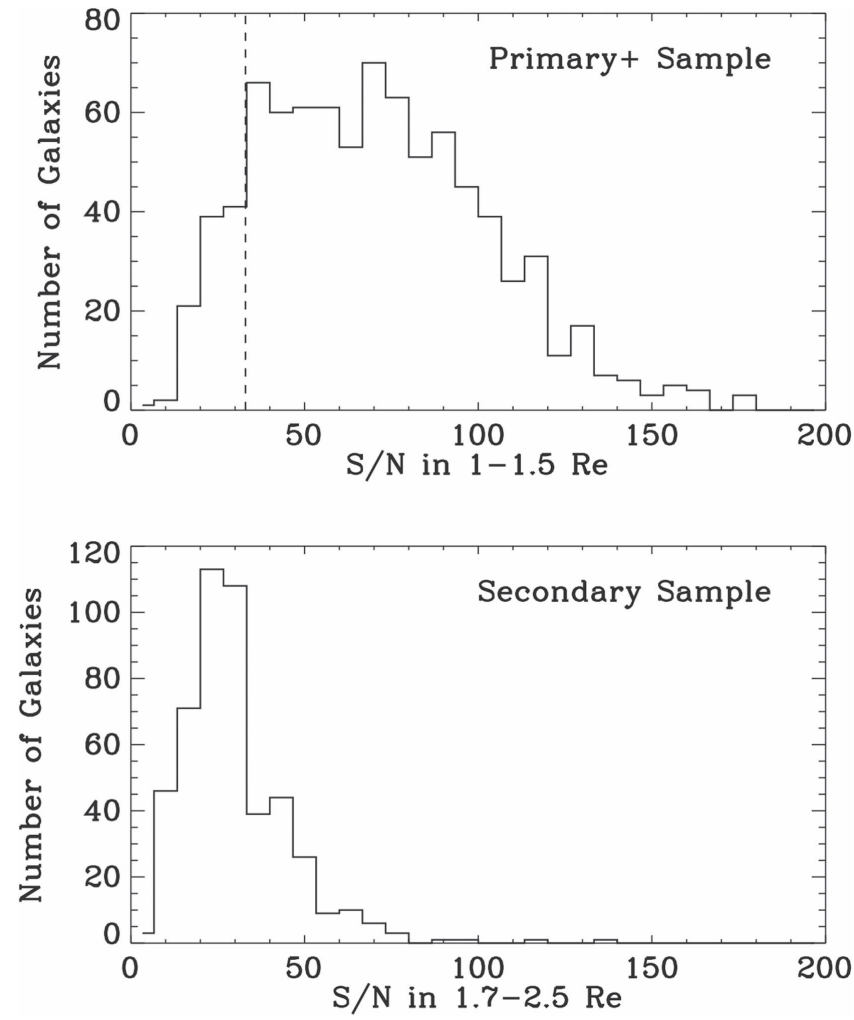

Figure 12. Upper panel: distribution of the stacked $\mathrm{S} / \mathrm{N}$ per $1.4 \AA$ pixel in $r$ band in the outer tertile of all Primary+ galaxies observed in the first year. This is the $\mathrm{S} / \mathrm{N}$ in the stacked spectra in an elliptical annulus between 1 and 1.5 effective radii of the galaxy or between two-thirds and 1 effective radius of the bundle, whichever is smaller. The vertical dashed line indicate the requirements. Lower panel: distribution of stacked $\mathrm{S} / \mathrm{N}$ in the outer tertile $\left(1.7 R_{e}-2.5 R_{e}\right.$, or $2 / 3-1$ effective bundle radius, whichever is smaller) of all Secondary galaxies.

reaches this $\mathrm{S} / \mathrm{N}$. The bottom panel shows the distribution for the Secondary sample for $1.7 R_{e}-2.5 R_{e}$, or between two-thirds and 1 effective bundle radius, whichever is smaller. About $30 \%$ of the Secondary sample reach an $r$-band $\mathrm{S} / \mathrm{N}$ per pixel greater 


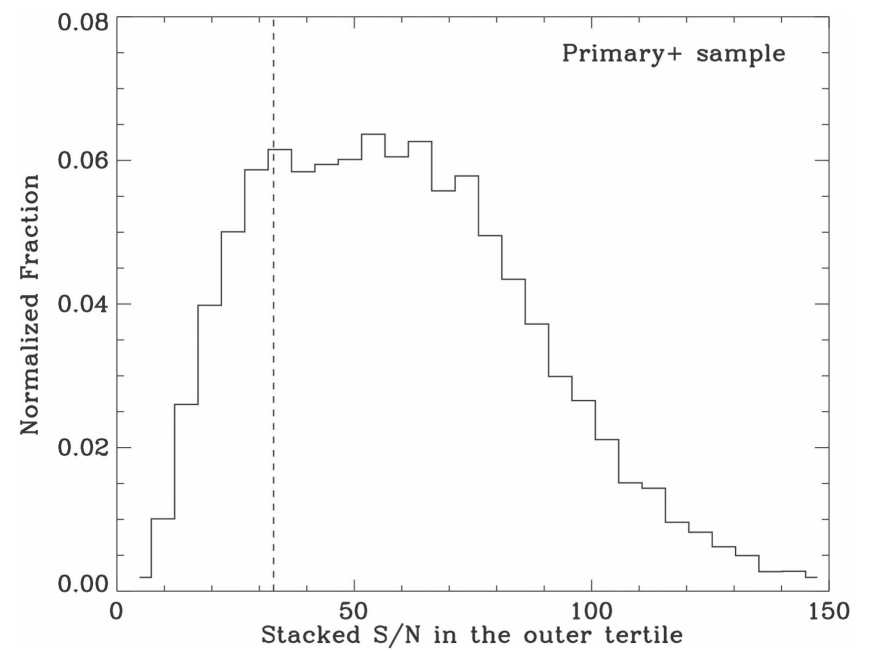

Figure 13. Projected final $\mathrm{S} / \mathrm{N}$ distribution in stacked spectra in the outer $1 R_{e}-$ $1.5 R_{e}$ among the Primary+ sample we expect from $6 \mathrm{yr}$ of observation. The vertical dashed line marks the $\mathrm{S} / \mathrm{N}$ threshold required for at least $75 \%$ of the galaxies in the Primary+ sample.

than 33 in this elliptical annulus, and $75 \%$ of them have $\mathrm{S} / \mathrm{N}$ greater than 20 .

Figure 13 shows the expected final $\mathrm{S} / \mathrm{N}$ distribution in our sample in 6 yr. Among the Primary+ sample, $80 \%$ will have a stacked $\mathrm{S} / \mathrm{N}$ per pixel in $r$ band in the outer tertile greater than 33 , meeting the science requirements.

\subsection{Projection for the Number of Galaxies}

As of 2016 April 18, we have completed observations of 156 plates, including two commissiong plates observed in 2014 March. These plates contain more than 2550 unique targets. Figure 14 shows our progress with time compared with expectations. We are slightly behind schedule because of the overexposing of plates in the first season (see discussion in Section 5.2). Since then, we are progressing as expected and have made up a fraction of the shortfall.

Given the remaining time we have before summer of 2020 , we expect to finish 575-600 plates, yielding a final sample of $\sim 10,000$ galaxies. The planned footprint is presented in Figure 4.

\section{DATA QUALITY ASSESSMENT}

In this section, we provide critical information about the data and an assessment of data quality. In a companion paper, Law et al. (2016), we describe the DRP in detail and also provide additional analysis of data quality. Law et al. provide examples of the reduction quality on individual plates. Here we present the overall statistics of quality metrics for the data set observed in the first year.

\subsection{Example Spectra from Data Cube}

Before we present the metrics, we illustrate the data quality obtained by MaNGA with two typical spectra from a spiral galaxy in Figure 15. One spectrum is from the central $0.5 \times 0.5 \operatorname{arcsec}^{2}$ spaxel in this galaxy, and the other is from an edge spaxel that is $13^{\prime \prime}$ away from the center. The central

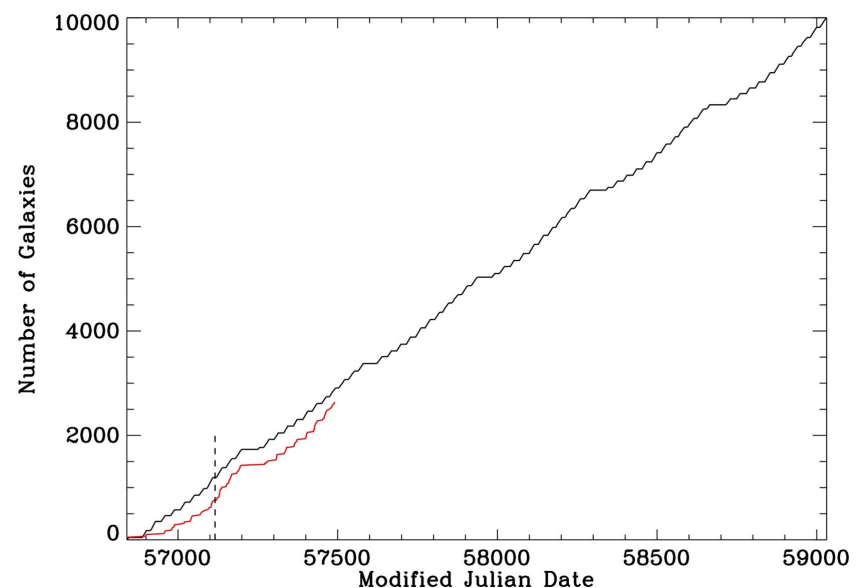

Figure 14. Current progress toward the final goal of 10,000 galaxies. The black line shows the expected number of plates as a function of date, and the red line shows the actual number of plates observed. MaNGA is slightly behind schedule because of bad weather and overexposing of plates in the first season. The dashed line marks the date when exposure times were corrected. Since then, we have been on track with expectations and have made up a fraction of the shortfall.

spectrum has an $\mathrm{S} / \mathrm{N}$ per pixel of $200-250$ in the $r$ band, and the edge spectrum has an $\mathrm{S} / \mathrm{N}$ per pixel of 20-30. No smoothing has been applied to either of the spectra. One could easily identify multiple spectra features in both spectra, such as $\mathrm{Ca}$ II $\mathrm{H} \& \mathrm{~K}, G$ band, $\mathrm{H} \beta, \mathrm{Mg}$ I b, $\mathrm{Na}$ I D, $\mathrm{H} \alpha$, [N II] $\lambda \lambda 6548,6583$, [S II] $\lambda \lambda 6716,6731$, and Ca II triplet. The two spectra have very different $D_{n}(4000)$, indicating different stellar population ages. They also display very different emission-line ratios in $[\mathrm{N} \mathrm{II}] / \mathrm{H} \alpha$, indicating that different ionization mechanisms are at play at these two locations.

\subsection{Resulting PSF on the Focal Plane from Seeing and Guiding}

The PSF is a critical element in the analysis of IFS data. Here, by PSF we mean the intrinsic light profile of a point source on the focal plane of the telescope. Because we are doing imaging spectroscopy, the knowledge about the PSF is critical for most of the analysis of the data. For an individual exposure, flux incident on a fiber bundle is spatially undersampled by the fibers and is not completely covered due to gaps between fibers. Thus, the IFU bundles alone cannot provide an accurate measurement of the PSF shape, but they can provide a refinement of the scale of the PSF if the shape is known. The guider images can provide such an initial measurement of the PSF shape. For a more detailed description of the guider system, see Section 5.6.

The science exposures are 15 minutes long, during which the guider system monitors the position of the guide stars relative to the position of the guide fibers. As described in Section 5.6, the guiding is not perfect and introduces some smearing of the integrated PSF over the 15-minute exposure.

To measure the integrated PSF, we bias-subtract and flatfield all the guider images and then stack the guider images taken during a science exposure. Figure 16 shows a stacked image made from 37 individual guider frames taken during a 15-minute exposure. This gives the time-integrated PSF. The 


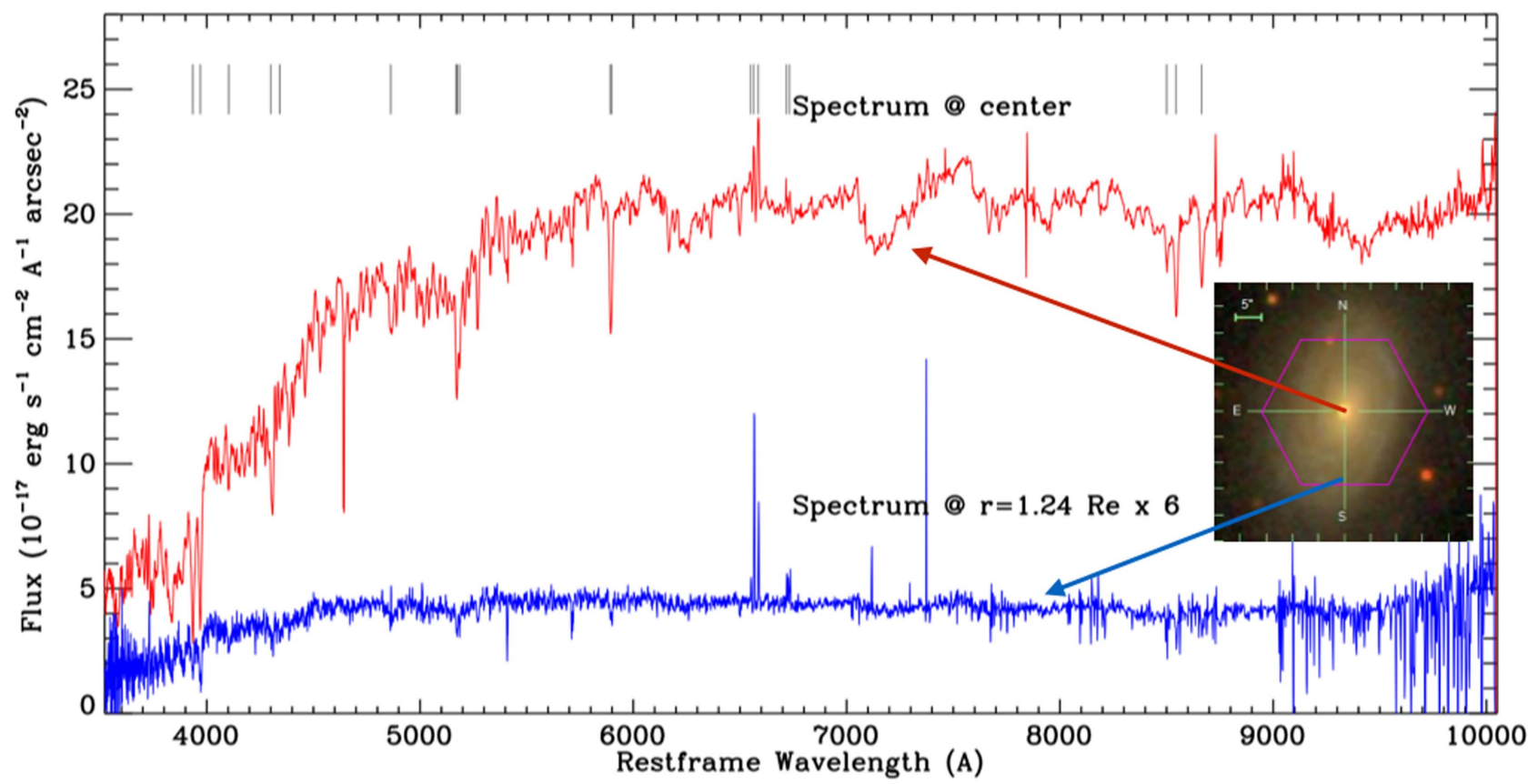

Figure 15. Example spectra from a typical MaNGA data cube. This galaxy was observed with IFU 12704 on plate 8138 . The inset shows the SDSS color image with the hexagonal IFU footprint overlayed. The top spectrum is from the central spaxel; the bottom spectrum is from $1.24 R_{e}$ away from the center and is multiplied by a factor of 6 for easier comparison with the central spectrum. No smoothing has been applied. Even the outer spectrum, which is fainter by a factor of 30 in $r$-band flux, has sufficient $\mathrm{S} / \mathrm{N}$ to clearly detect numerous spectral features, which are marked with the short lines on top. The sharp spikes in the near-IR, particularly in the bottom spectrum, are due to sky subtraction residuals. Note that the two spectra have very different shapes and feature emission lines with significantly different strengths relative to the continuum.

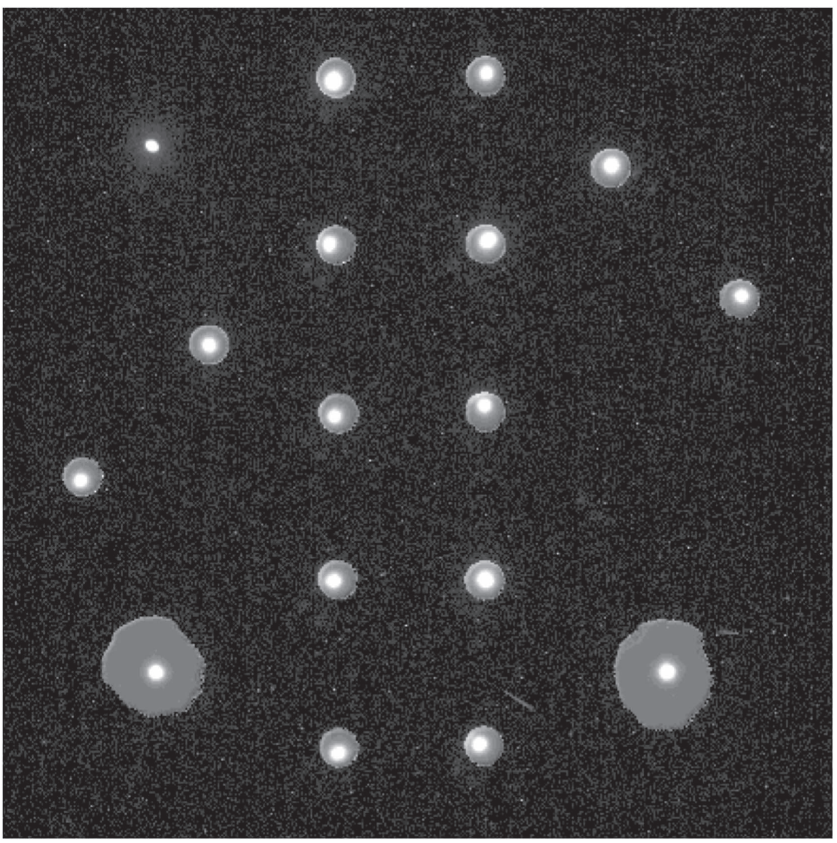

Figure 16. Example stacked guider image made from co-adding 37 individual flat-fielded guider frames taken during a 15-minute exposure. The 16 guide fibers are positioned in this particular configuration on the fiber output block that is imaged by the guide camera. The bright dot in the upper left is a Tritium spot, which can be used to check the focus of the camera. The two larger guide fibers near the bottom left and bottom right are acquisition fibers. They provide a larger area for us to measure the sky background.

left panel of Figure 18 shows the distribution of seeing FWHM observed for all exposures by the guider during the first year of observations. The median seeing is $1 . " 50$, and the range is $1^{\prime \prime}-2 ! " 5$.
In our DRP, we model the focal-plane PSF seen by the fiber bundles with a double-Gaussian function:

$$
F(r)=A_{1} \exp \left(-\frac{r^{2}}{2 \sigma_{1}^{2}}\right)+A_{2} \exp \left(-\frac{r^{2}}{2 \sigma_{2}^{2}}\right) .
$$

We prefer to model the PSF as a double Gaussian rather than a Moffat because Gaussians are much faster to integrate over an aperture $^{46}$ than Moffat functions and Moffat functions provide only moderate improvements. Figure 17 shows a typical PSF measured by the guider with a 1"! 5 FWHM. A double Gaussian provides an adequate description of the central region but misses the extended tail beyond 3". A Moffat function only does slightly better and cannot fit the extended tail either. The extended tail contains about $3 \%$ of the total flux. If one is concerned with features in MaNGA galaxies that involve large surface brightness contrasts, this issue may be important.

The PSF seen by the guider may be slightly different from that seen by the fiber bundles. This is because the guider system modifies the PSF in two ways. First, cross-talk between individual fiber strands in an imaging fiber can smear the PSF. Second, small focus offsets in the imaging camera could also modify the PSF.

To address the impact of these effects, we refine the scales of the PSF, using mini-bundle observations of standard stars, assuming that the shape of the PSF is the same as seen by the

\footnotetext{
${ }^{46}$ We need the computation to be fast because we need to compute many fiber-convolved PSFs with different sizes during the flux calibration step (Yan et al. 2016). The integration of the Moffat function does not have an analytic formula, and we have to integrate numerically, which is very slow. Integration of the Guassian function is easy to compute using the error function, and no numerical integration is required. Therefore, they differ by orders of magnitude in computation time.
} 


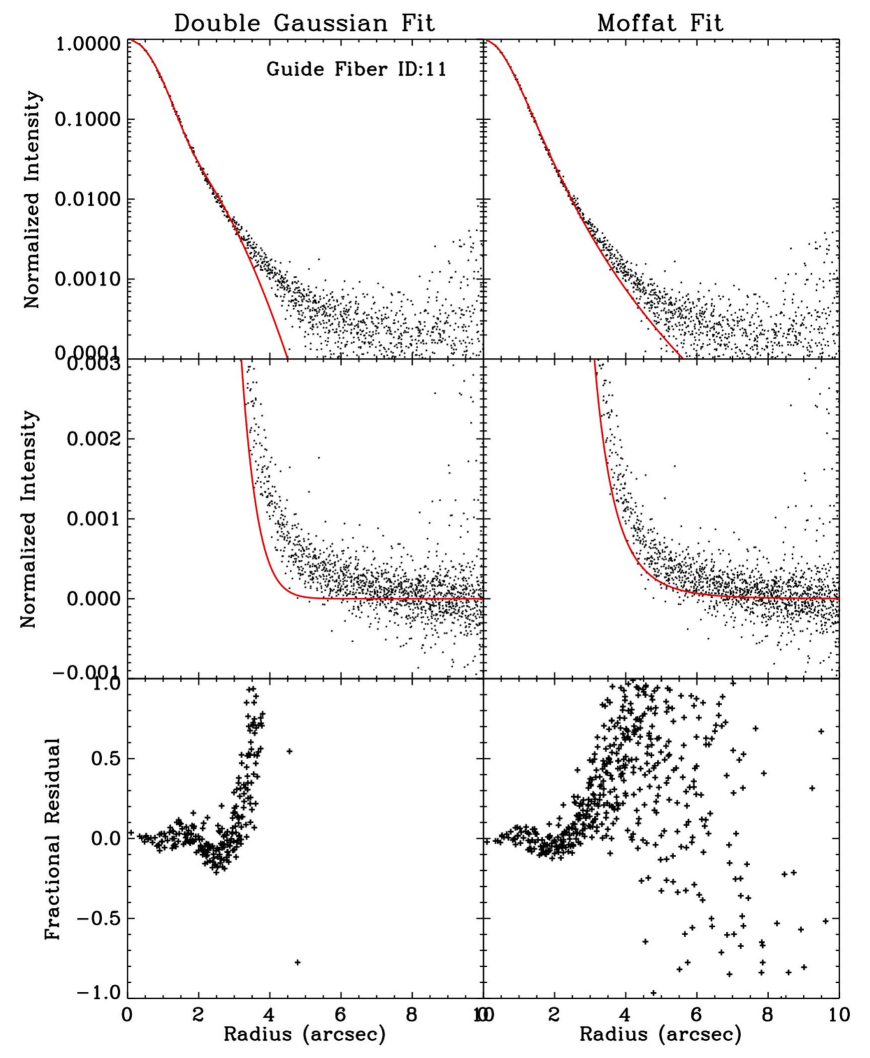

Figure 17. Spatial profile of an example guide star image fitted by a doubleGaussian model (left) and a Moffat model (right). The $y$-axes of the top four panels are the flux ratio relative to the peak flux of the best-fit model. The top panels show the whole curve in log units, in which negative points are excluded. The middle panels show the fit in linear units zoomed in around the low flux outskirts. The bottom panels show the fractional residual relative to the model. There is an additional tail beyond $3^{\prime \prime}$ that cannot be adequately accounted for by either the double-Gaussian model or the Moffat model. The tail contains about $3 \%$ of the total flux.

guider. For flux calibration purposes, we observe 12 F-type subdwarfs on each plate simultaneously with the science targets. By fitting for the flux ratios among fibers in the minibundle, we find that on average the PSF seen by the IFU bundles is $90 \%$ in width compared to the guider PSF.

\subsubsection{What PSF Should I Use?}

First, we would like to make a distinction between the "PSF" and the "fiber-convoled PSF." By "PSF," we mean the intrinsic light profile produced by a point source on the focal plane of the telescope. By "fiber-convolved PSF," we mean this PSF convolved with a top-hat $2^{\prime \prime}$-diameter fiber aperture. When using the fiber bundles to observe a star, the flux in each fiber is equal to a sampling off this fiber-convolved PSF at the center position of that fiber.

What PSF should be used in the analysis of the MaNGA data depends on what data products are used in the analysis, the data cubes or the RSS files.

If one uses the datacubes, one should use the "reconstructed PSF" stored along with the data cube. The data cube is produced in the $3 \mathrm{~d}$ stage of the DRP using an image reconstruction algorithm, the Modfied Shepard method, to produce a final image on a regular grid of $0.5 \times 0$ ". 5 square spaxels. The input to this algorithm includes the fiber spectra and the relative positions of all fibers in a bundle at all wavelengths, from all exposures. Suppose one observes a star, and the fiber flux is equal to a sampling of a "fiber-convolved PSF." Therefore, to obtain a corresponding PSF to the data cube, we process the "fiber-convolved PSFs" of all contributing exposures through the same image reconstruction algorithm, applying exactly the same offsets as applied to all exposures (see Law et al. 2016). This produces a "reconstructed PSF." To use this PSF, one should not do any more integration with either the $0 . " 5$ spaxel or with the fiber aperture, as they are already included.

In the middle panel of Figure 18, we show that the FWHM of the reconstructed PSF is well correlated with the median seeing of all the exposures going into a data cube. The right panel of this figure shows the final FWHM distribution of this "reconstructed PSF" among all galaxies completed in the first year. The median FWHM is 2 !" 54, with a tail extending to 2 ". 8 . This is the final angular resolution of the data cube.

If one uses the RSS files, which store the spectra per fiber per exposure, one would need to compute the PSF associated with each exposure. In this case, one should start with the guider image, measure the PSF (intrinsic light profile on focal plane modeled as double Gaussian or Moffat function), convolve with the $2^{\prime \prime}$ fiber aperture, and scale down by $10 \%$ in width to get the per-exposure "fiber-convolved PSF." Note that the PSF varies across the focal plane and varies with wavelength. Thus, one needs to adjust it according to the procedure as described by Yan et al. (2016) before the fiber aperture convolution. If one uses the RSS file or applies forward modeling to fit the data, this is the PSF to use.

\subsection{Sampling Uniformity from the Actual Dithers}

Here we evaluate our dithering accuracy. In Section 5.1, we defined the dithering uniformity statistic $(\Omega)$ to be the maximum offset between dithers in a set. There are two parts contributing to this offset: one is due to atmospheric refraction and the optical distorion of the telescope, and the other is due to imperfect guiding. The former is theoretically predictable, but the latter is not. We can measure the offset due to imperfect guiding by matching the data to SDSS imaging, as mentioned above. This is done in the extended astrometry module of the DRP (Law et al. 2016). Combining both components, we directly measure $\Omega$ for all of our galaxies. Figure 19 shows the Omega distribution for all galaxies observed in the first year. The offset is largest at the bluest wavelength we cover. At $3622 \AA, 98.6 \%$ of all galaxies have $\Omega<0$ " 4 . By meeting this requirement, we ensure a high degree of spatially uniform sampling as a function of wavelength across the full wavelength range.

\subsection{Spectral Resolution}

Figure 20 shows the distribution of the obtained spectral resolution as a function of wavelength for all 1390 galaxies observed in the first year. As discussed above, the focal plane is not flat at the CCD. Thus, there are variations in instrumental dispersion as a function of both slit position and wavelength. The variation is large in the blue camera because its focal plane is strongly curved in the spatial direction. There can also be large variations in resolution within a single IFU, especially if the IFU is placed close to the edges of the slit, where the focal plane has the steepest slope relative to the CCD. The arc frame provides the basic measurement of the instrumental resolution. 

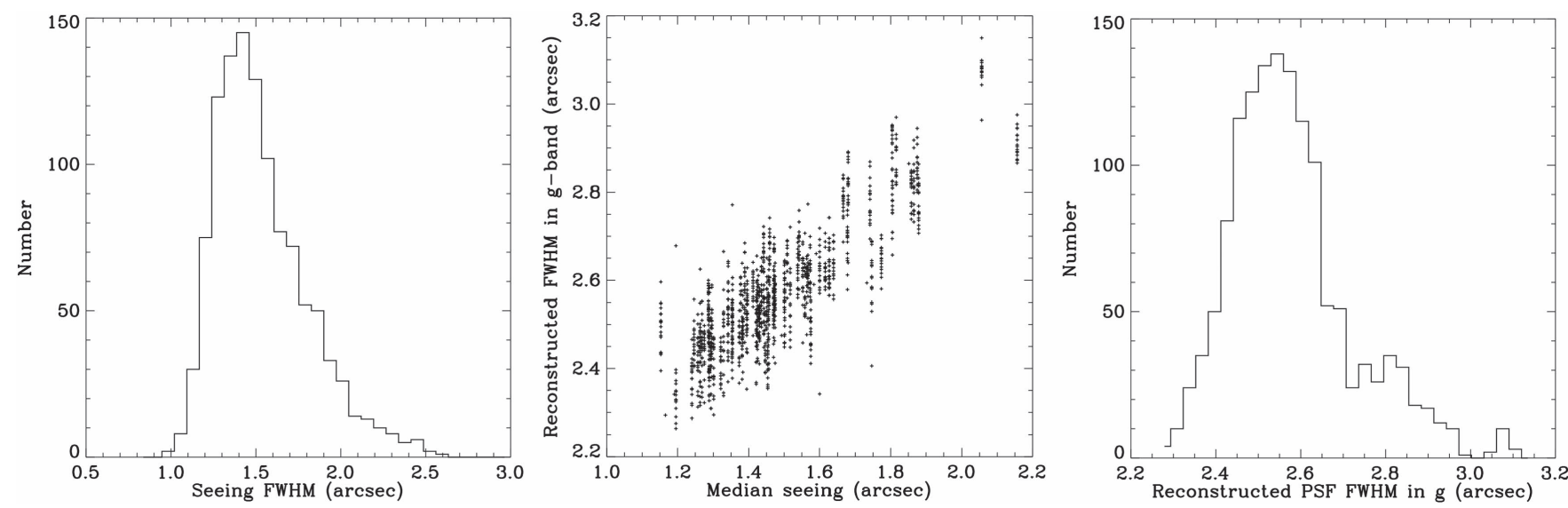

Figure 18. Left: seeing distribution of all MaNGA science exposures taken in the first year of operation. Middle: median intrinsic per-exposure seeing FWHM vs. FWHM of the reconstructed PSF in the data cube in $g$ band for all galaxies completed in the first year. Right: distribution of the FWHM of the reconstructed PSF in $g$ band.

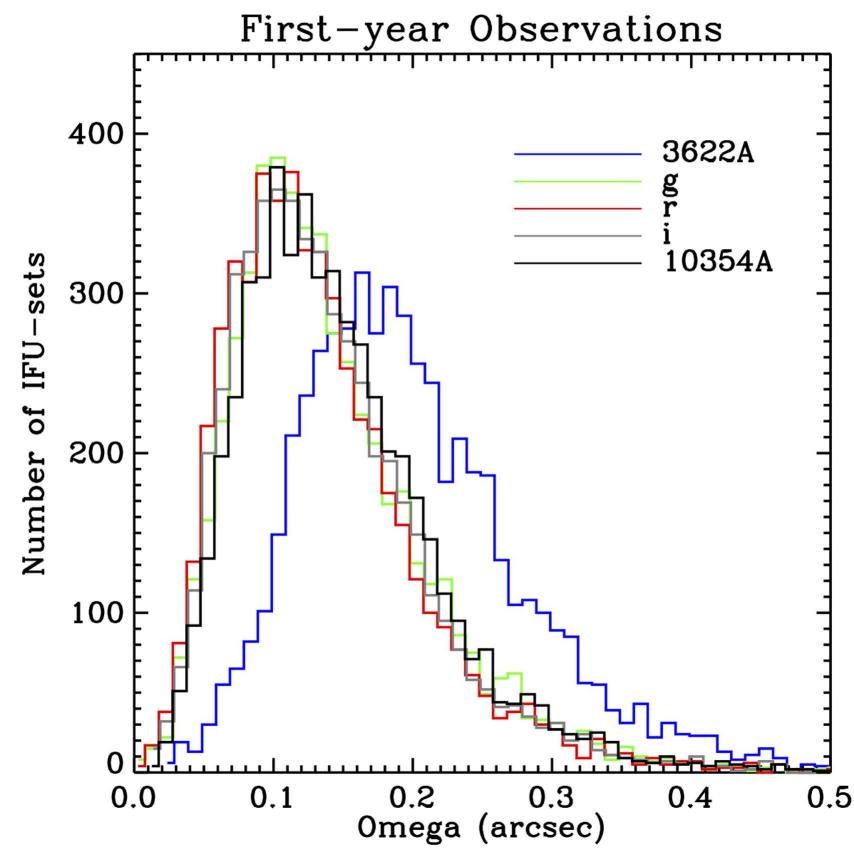

Figure 19. Distribution of the maximum $\Omega$ offsets (see Section 5.1) among dithers in a set for all IFUs targeting galaxies in the first year of observation. The different histograms show the offsets at five wavelengths: including the effective wavelengths of the $g, r$, and $i$ bands, and the bluest and reddest wavelengths we cover. The offset is largest at the bluest wavelengths due to the large chromatic differential refraction in the blue.

Two other factors also change the instrumental line-spread function (LSF) in the science exposures compared to the arc exposures. The science exposures are 15 minutes long. Over this 15-minute integration time, as the telescope tracks the field, there can be small focus drifts and small detector movements due to instrument flexure. These give a slight, additional broadening to the LSF. Since we often take flats and arcs at both the beginning and the end of the exposure sequence, we found that the cameras in spectrograph 2 (b2 and $\mathrm{r} 2$ ) can have significant focus drifts over a few hours. This is illustrated in Figure 21, which compares the instrumental dispersion as a function of fiber ID for the four cameras between two arc exposures separated by $4.8 \mathrm{hr}$. Significant changes are apparent in the edge fibers in b2. Additionally, the detector can experience lateral movements in both the spatial and spectral

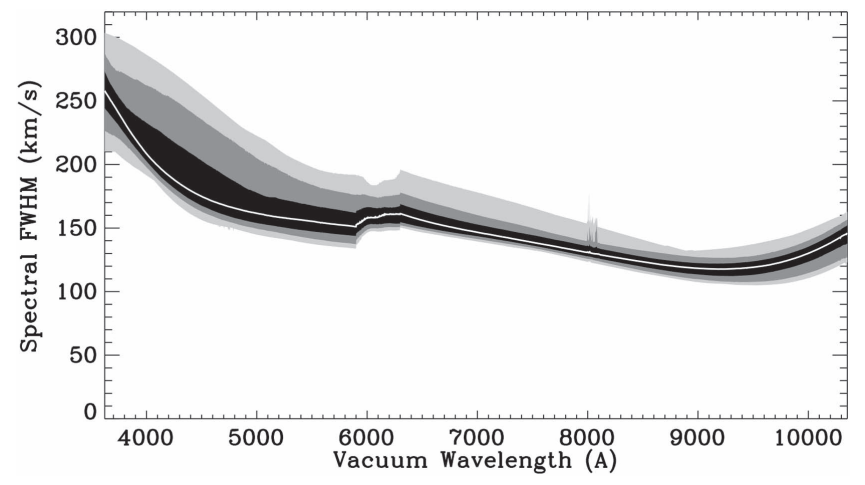

Figure 20. The white curve shows the median instrumental resolution expressed in FWHM in velocity units as a function of wavelength among all 1390 galaxies to be released in DR13. The black region indicates the 15.85 to 84.15 percentiles of the distribution at each wavelength, which is roughly $\pm 1 \sigma$ around the median. The dark-gray zone indicates the 2.5 to 97.5 percentiles, and the light-gray zone indicates the 0.15 to 99.85 percentiles of the spectral dispersion. The region between 5900 and $6300 \AA$ is where the blue cameras and red cameras overlap in wavelength. The dispersion here is averaged between the two cameras. The feature around $8000 \AA$ is due to the middle three rows of the red detector having slightly different pixel widths. The velocity FWHM presented here does not include the $10 \%$ broadening described in the text, i.e., the true values are about $10 \%$ larger than those shown here.

directions at a small fraction of a pixel. Flexure tests show that at an altitude of $60^{\circ}$, over $360^{\circ}$ of rotation, the min-to-max lateral shift in the spatial direction can be as large as $0.4-0.6$ pixels and in the spectral direction can be as large as $2-3$ pixels. When we track on the sky, over 15 minutes, the change in gravity vector is much smaller, but there still can be small subpixel shifts that will smear the LSF slightly. The overall shift between the arc frame and the science frame is taken out in the reduction pipeline by shifting the wavelength solution to match the sky lines. In the pipeline, we use the measured strong sky line widths to modify the LSF and report a final instrumental resolution that includes the above factors. The reported LSFs in the data released in DR13 and shown in Figure 20 are these final sky-line-matched dispersion values (Law et al. 2016).

The SDSS Legacy software assumes that the LSF is a Gaussian before pixel integration, i.e., the flux in a pixel equals the integration of a Gaussian profile over the pixel width. This is different from assumptions made in many other analyses 

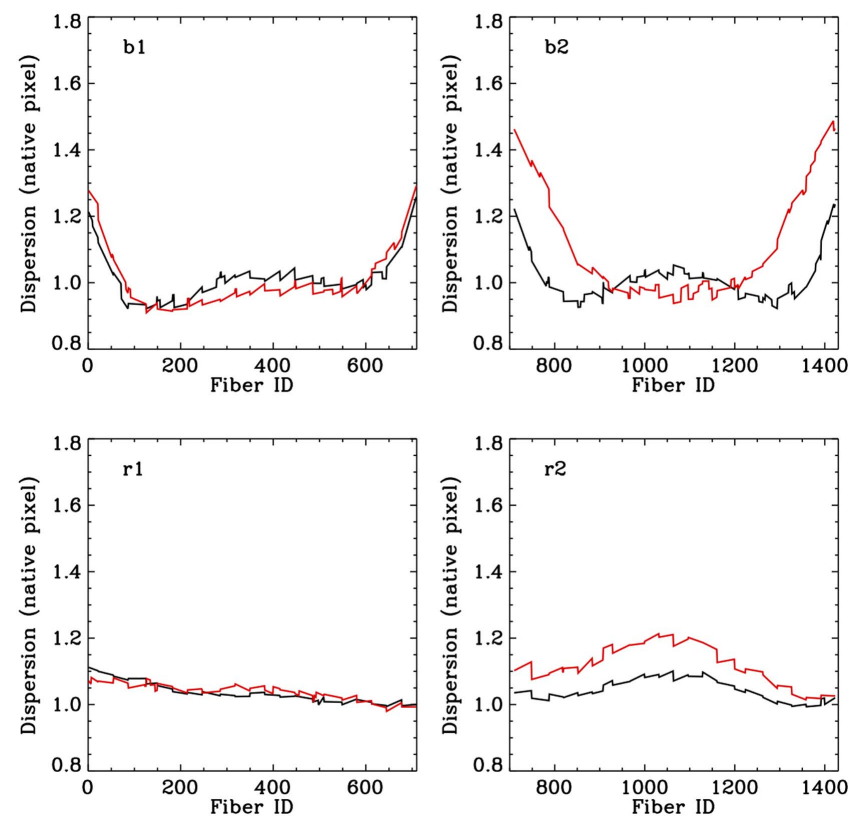

Figure 21. Comparison of the instrumental line dispersion ( $\sigma$ in native pixel units) in the four cameras as a function of fiber ID for Row 2000 (middle of detectors) for two arc frames (black vs. red lines) separated by $4.82 \mathrm{hr}$. There is significant focus drift in $\mathrm{b} 2$ and $\mathrm{r} 2$, causing the line dispersion to change significantly. The effect is strongest in b2 near the edges of the slit. This effect is taken into account in the delivered instrumental resolution by matching to the widths of sky lines.

that assume that the flux in a pixel equals the value of a Gaussian profile sampled at the center of the pixel. The LSF is critically sampled (FWHM $\geqslant 2$ pixel width) by the native pixel width in most parts of our detectors. In this regime, these differing assumptions can cause a $4 \%$ width difference in the measured LSF. In addition, when we resample the spectra from the native pixel sampling to a regularly spaced linear or logarithmic sampling, we effectively broaden the LSF. Combined together, these two factors cause a roughly $10 \%$ increase in LSF or equivalently a $10 \%$ decrease in spectral resolution. This factor is not included in the data released in DR13 but will be addressed in future data releases. See Law et al. (2016) for more details.

Our ability to measure velocity dispersion for stars and gas below the intrumental resolution depends sensitively on how well we measure the effective intrumental resolution. We test this by measuring the distribution of $\mathrm{H} \alpha$ line width in starforming galaxies observed in the first year, and we compare it with those measured using high-resolution spectroscopy. In the left panel of Figure 22 we show the $\mathrm{H} \alpha$ line width $(\sigma)$ distribution in local face-on star-forming galaxies measured by Andersen et al. (2006) using high-resolution spectra. The line widths are corrected for intrumental dispersion. The median value is around $18 \mathrm{~km} \mathrm{~s}^{-1}$. In the right panel of Figure 22, we show the intrinsic $\mathrm{H} \alpha$ line width distribution measured in MaNGA spectra. The line widths are also corrected for instrumental dispersion. The two histograms shown use different estimates of the instrumental dispersion. The pink histogram uses the instrumental dispersion reported by the DR13 version of the pipeline directly, while the blue histogram broadens the instrumental dispersion by $10 \%$ before using, as described above. The latter version yields an intrinsic $\mathrm{H} \alpha$ line width distribution with a median around $26 \mathrm{~km} \mathrm{~s}^{-1}$, much closer to the value measured by high-resolution spectroscopy. There is still a small difference, which means that we may still be underestimating the instrumental dispersion $\left(\sim 69 \mathrm{~km} \mathrm{~s}^{-1}\right)$ slightly, by about $3 \%$. This means that we would have at most a $10 \%$ bias on velocity dispersion around $40 \mathrm{~km} \mathrm{~s}^{-1}$, which is $40 \%$ lower than our instrumental resolution.

\subsection{Quality of Sky Subtraction}

One unique advantage of MaNGA is its very wide wavelength coverage, especially the coverage in the near-IR, which includes many spectral features important for interstellar medium, stellar population, IMF, and kinematics diagnostics. However, the red part is full of the atmospheric emission lines. Reliable sky subtraction is critical to take advantage of this region of the spectra. Law et al. (2016) give extensive details on how we perfrom sky subtraction and a detailed assessment of the quality. Here we briefly summarize the result. Using specially built plates on which all fibers point at empty sky locations ("all-sky plates"), we tested our sky subtraction. We found that the residuals in our sky-subtracted sky spectra have a distribution that is very consistent with the expected uncertainty due to read noise and Poisson counting statistics. For individual wavelengths, the residuals are consistent with Poisson expectations in line-free regions of the spectra and are slightly above Poission expectation in strong-line regions. Using all-sky plates, we have verified that the sky-subtracted sky fibers have no significant systematic residuals. This is done by stacking a large number of these residual spectra and verifying that the rms of the stack decreases following the expectation of Poisson statistics.

Here we present an evaluation of the sky subtraction accuracy in every science plate using the sky fibers. We take the standard deviation of the residual in the sky-subtracted sky spectra and then divide it by the expected uncertainty given by read noise and Poisson statistics, resulting in what we call "the Poisson ratio." We evaluate this Poisson ratio for four wavelengths in the spectra: two are centered on moderately strong sky lines ( $5462 \AA$ in the blue and $8888 \AA$ in the red), and two are on line-free continuum regions $(5500 \AA$ in the blue and $6800 \AA$ in the red). Figure 23 shows the distribution of this Poisson ratio for all exposures taken in the first year. Because the sky model is built from the sky fibers, evaluating the Poisson ratio using the sky fibers underestimates the actual Poisson ratio. Using 14 exposures taken on multiple all-sky plates, we compare the Poisson ratio between science fibers and sky fibers for these four wavelengths. We found that the science fibers show larger Poisson ratios than sky fibers by different factors on these different wavelengths: $2 \%$ at $5500 \AA$, $7 \%$ at $6800 \AA, 12 \%$ at $5462 \AA$, and $15 \%$ at $8888 \AA$. Therefore, in Figure 23 we have scaled up the Poisson ratio by these factors. The subtraction is very close to Poisson in the continuum and slightly above Poisson around sky emission lines.

\subsection{Quality of Flux Calibration}

As described in detail by Yan et al. (2016), our flux calibration algorithm is different from single-fiber spectroscopy surveys because we are performing imaging spectroscopy. We would like to only correct for the flux lost due to the imperfect system response and atmosphere extinction, but not for any flux lost due to the limited aperture of each fiber. The 

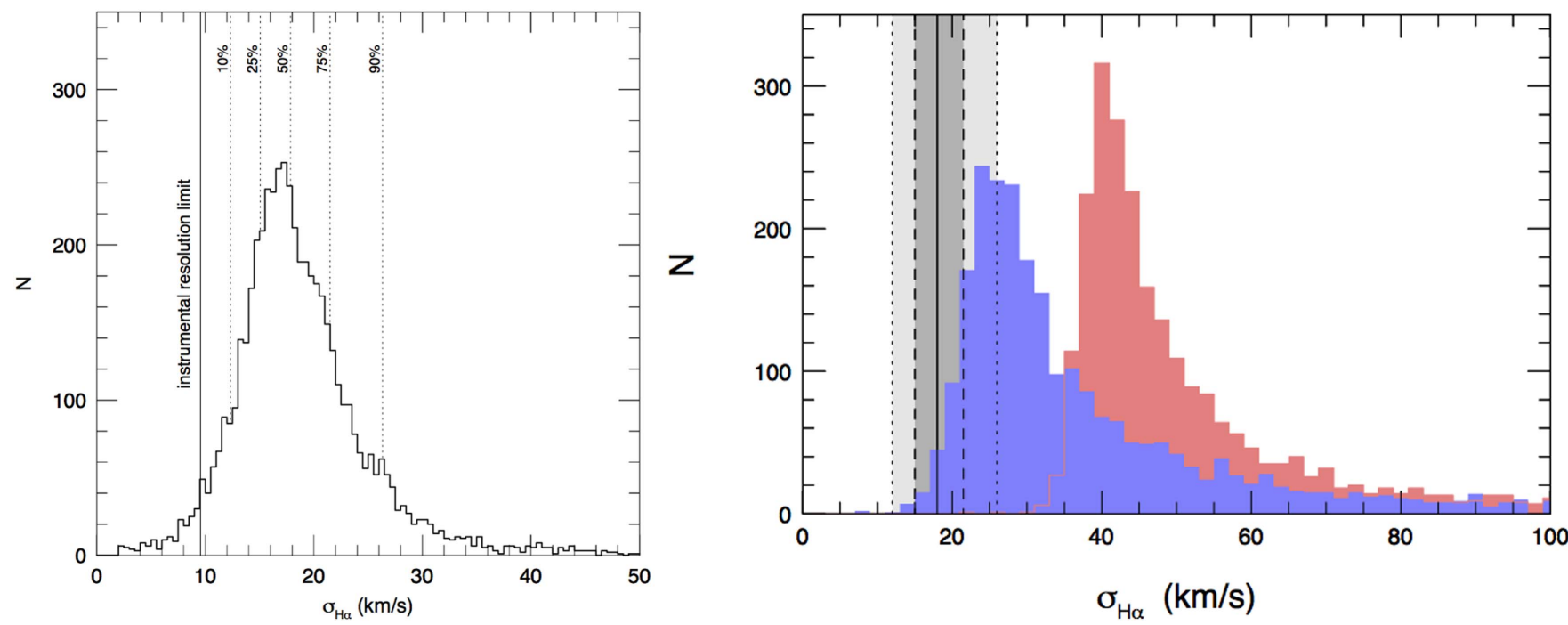

Figure 22. Left: distribution of intrinsic $\mathrm{H} \alpha$ line width among star-forming disk galaxies observed by Andersen et al. (2006) with high-resolution spectroscopy. Right: distribution of intrinsic $\mathrm{H} \alpha$ line width measured in star-forming disk galaxies in MaNGA data before (pink histogram) and after (blue histogram) making a 10\% correction to the instrumental dispersion. The vertical lines indicate the 10th, 25th, 50th, 75th, and 90th percentiles of the distribution in the Andersen et al. (2006) sample. The distribution in MaNGA galaxies after correction has a median that is much closer to, but still higher than, that measured by high-resolution spectroscopy. This means that we know our actual instrumental dispersion to better than $3 \%$ accuracy.
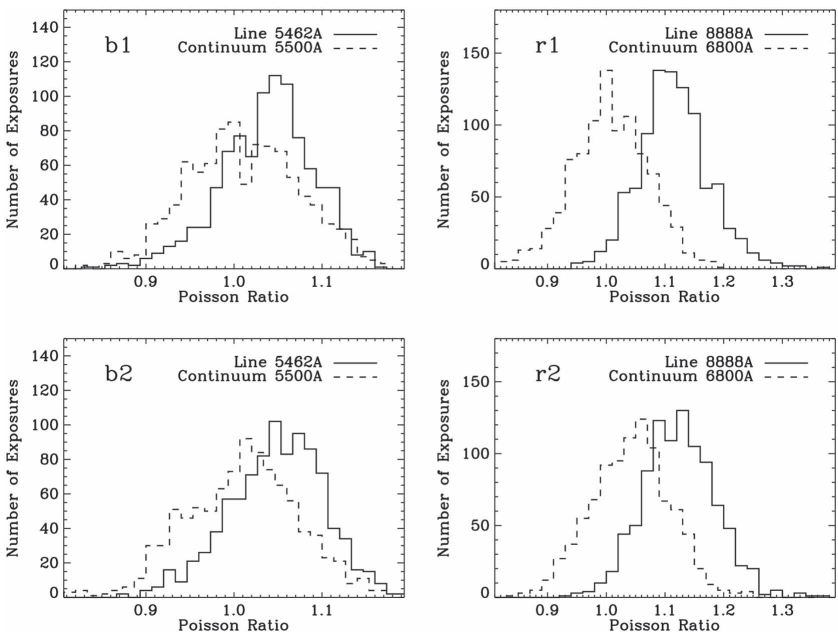

Figure 23. Poisson ratio distribution among all exposures taken in the first year, for different wavelengths in the four cameras. The Poisson ratio indicates how the distribution of the residual noise compares to the expected uncertainty given by read noise and the Poisson counting statistics. Each panel shows one camera. The solid histograms show the Poisson distribution around moderately strong sky emission lines; the dashed histograms show the Poisson ratio distribution around line-free continuum regions. The wavelengths are indicated in the legend. The sky subtraction is very close to Poisson in the continuum and slightly above Poisson around emission lines.

separation of the two flux loss factors is achieved by modeling a star's flux as received by the seven fibers in a mini-bundle. Given an initial guess of the PSF provided by the guider, we use the flux ratios among the seven fibers to constrain the exact position of the star relative to the bundle, the size of the PSF, and the level of differentiatial atmosphere refraction. With this spatial model accounting for the aperture-induced flux loss, we can then estimate the flux loss due to the system response. We target 12 stars per plate with six per spectrograph. The average of six stars per spectrograph provides the throughput correction that is applied to all galaxy fiber spectra.
In Yan et al. (2016), we provided two assessments of the flux calibration accuracy. From comparison to broadband imaging of galaxies, we showed that the relative calibrations in $g-r$, $r-i$, and $i-z$ colors are good to $\pm 3 \%$. From the comparison between completely independent measurements of the throughput curves, we showed that we achieve better than 5\% absolute calibration for $89 \%$ of the wavelength range and achieve a relative calibration rms of $1.7 \%$ between $\mathrm{H} \alpha$ and $\mathrm{H} \beta$ and $4.7 \%$ between [N II] $\lambda \lambda 6548,6583$ and [O II] $\lambda 3727$ (Yan et al. 2016).

Here we provide yet another evaluation of the flux calibration accuracy based on comparison of repeated galaxies. These galaxies are observed on different plates with different standard stars. Therefore, the observations and calibrations are completely independent of each other. We stack the spectra from the data cubes in a $5^{\prime \prime}$ radius circle around the center of each object. In Figure 24, we show an example pair of these repeated observations of one galaxy. The bottom panel shows the ratio of the two spectra as a function of wavelength. The two spectra agree to within a few percent, and not more than $10 \%$ at the wavelength extremes. In Figure 25 we show the ratio plots for 32 pairs of repeated observations. In most cases, the ratio is very flat and is very close to 1 . Sometimes, the absolute calibration of the two observations could differ by $\pm 10 \%$, but the relative calibration is mostly flat.

\section{VERIFICATION OF THE SCIENCE REQUIREMENTS}

In this section, we verify that the science requirements set forth in Section 2 are achieved with our first-year data.

\subsection{SFR Surface Density}

We require the SFR surface density to be measured to better than 0.15 dex precision when the SFR density is above $0.01 M_{\odot} \mathrm{yr}^{-1} \mathrm{kpc}^{-2}$ and $E(B-V)<0.5$. Given the derivation in Section 2.1, these limits correspond to an $\mathrm{H} \alpha \mathrm{SB}$ of $6.58 \times 10^{-17} \mathrm{erg} \mathrm{s}^{-1} \mathrm{~cm}^{-2} \operatorname{arcsec}^{-2}$ and an $\mathrm{H} \beta \mathrm{SB}$ of $1.36 \times 10^{-17} \mathrm{erg} \mathrm{s}^{-1} \mathrm{~cm}^{-2} \operatorname{arcsec}^{-2}$. We evaluate the accuracy 


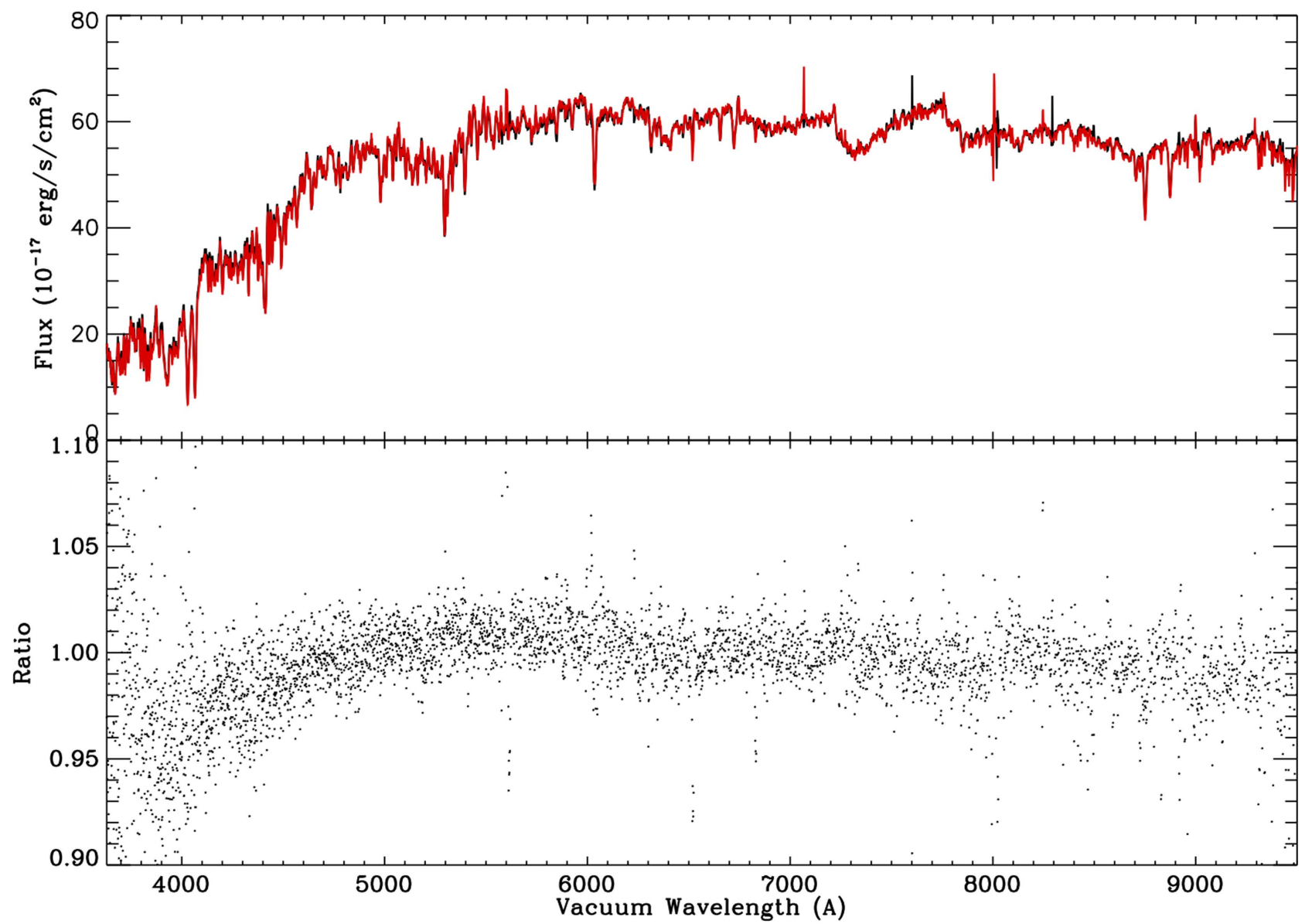

Figure 24. Top: comparison of the stacked spectra for the same galaxy observed on two different plates. Bottom: ratio between the two stacked spectra illustrating a flux calibration uncertainty better than $5 \%$.

of these line measurements at this surface brightness using repeated observations.

The reduced data cubes are processed by our dedicated data analysis pipeline (K. Westfall et al. 2016, in preparation). Briefly, the emission lines are measured in the reduced data cube for each spaxel after the subtraction of the stellar continuum. We fit the emission-line-only spectra with Gaussians around the lines, using multiple Gaussians when necessary (e.g., $\mathrm{H} \alpha+[\mathrm{N}$ II] triplet). For the comparison between repeated observations of the same galaxies, we first smoothed the emission-line flux map by a $2 . " 5 \times 2 . .5$ square kernel, which is equivalent to summing the flux in a resolution element. Then, we take the difference in line flux between the two independently measured flux maps of the same galaxy and then divide by their average. Figure 26 shows the fractional difference in line flux versus the average line flux. We can see that the fractional differences decrease with increasing flux. At the threshold surface brightness, we found that the fractional difference in $\mathrm{H} \alpha$ flux has an rms of $7.7 \%$ around 0 after one round of rejection of points more than $3 \sigma$ away from zero, and $\mathrm{H} \beta$ has an rms of $11.9 \%$. Since this is the difference of two independent measurements, the actual uncertainty on the measurement is a factor of $\sqrt{2}$ smaller, at $5.4 \%(\mathrm{H} \alpha)$ and 8.4\% $(\mathrm{H} \beta)$. According to Equation (12) in Yan et al. (2016), this would yield a final fractional uncertainty on SFR of $23.3 \%$ or $0.1 \mathrm{dex}$. In this calculation, we have included the $1.7 \% \mathrm{rms}$ relative calibration error between $\mathrm{H} \alpha$ and $\mathrm{H} \beta$ and the $4 \% \mathrm{rms}$ error in absolution calibration around $\mathrm{H} \alpha$ (Yan et al. 2016), which do not dominate the uncertainty. We have met the science requirement on the SFR surface density.

At lower SFR surface density, the uncertainty increases. At $0.003 M_{\odot} \mathrm{yr}^{-1} \mathrm{kpc}^{-2}$ and $E(B-V)<0.5$, the fractional uncertainty is about $50 \%$, or 0.2 dex. At $0.001 M_{\odot} \mathrm{yr}^{-1} \mathrm{kpc}^{-2}$, the uncertainty is $75 \%$, or $0.3 \mathrm{dex}$.

There are a small fraction of spaxels with much larger fractional error. The reasons for this are still under investigation. There also appears to be a systematic difference between $\mathrm{H} \alpha$ fluxes of repeated observations at high line fluxes, which is as large as $10 \%$. These cannot be caused by flux calibration error as the difference is not constant with changing flux. The actual cause is also to be investigated.

\subsection{Gas Metallicity Gradient}

Our science requirement on gas metallicity is to measure the gradient to better than $0.04 \mathrm{dex}$ per $R_{e}$. For all galaxies observed in the first year, we subtracted the stellar continuum from the spectrum in each spaxel, performed Voronoi binning based on the $\mathrm{H} \alpha \mathrm{S} / \mathrm{N}$, and then measured the emission fluxes in each bin, as done by Belfiore et al. (2016). We then classified the bins according to their positions on line ratio diagnostic diagrams. For all bins classified as star formation, we measure their gas-phase metallicity, using the R23 metallicity indicator (Tremonti et al. 2004). For measuring the gradient, we group the bins into a set of elliptical annuli. In each annulus, we measure the mean metallicity and the error of the mean using 


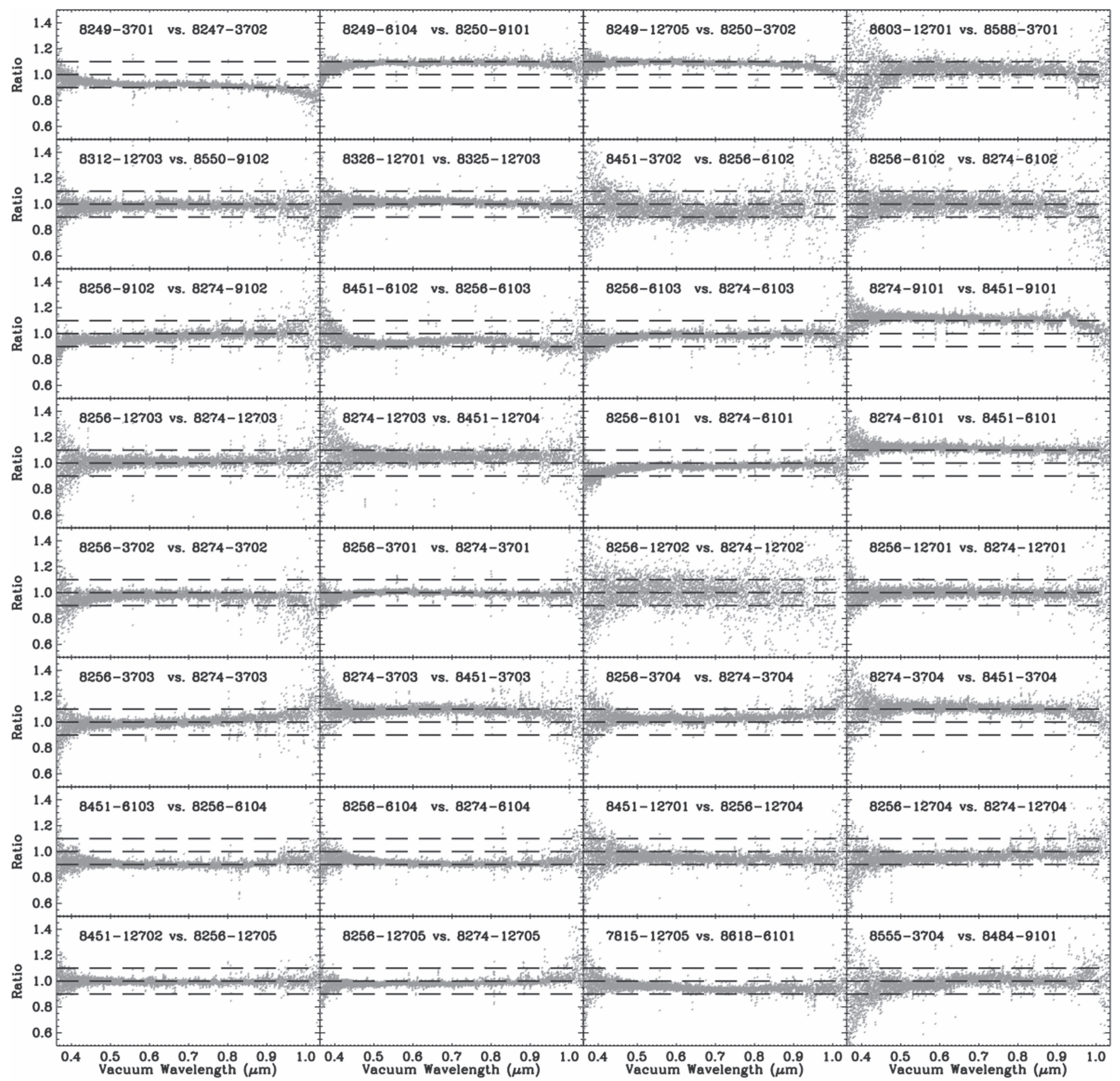

Figure 25. Flux ratio as a function of wavelength between two independent observations of the same galaxy. The three dashed horizontal lines in each panel mark unity and $\pm 10 \%$. In the great majority of cases, the ratios are flat with wavelength, indicating excellent quality in relative calibration. In some cases, the absolute calibration between two observations can differ by about $10 \%$.

the biweight estimator (Beers et al. 1990). Then we fit a linear function through all the annuli. The distribution of the derived uncertainty is shown in Figure 27. We have experimented using different numbers of annuli, and the results do not change statistically. For all star-forming galaxies, we found that $\sim 68 \%$ of them have a gradient error that is smaller than 0.04 dex per $R_{e}$. We met this science requirement for the majority of the sample.

Using a different metallicity indicator, such as O3N2 (Pettini \& Pagel 2004), gives similar results in the error distribution, but different gradients. There are certainly systematic errors associated with the metallicity calibration adopted (Kewley \& Ellison 2008). As stated in Section 2, our requirement is set only on the precision of the measurement. The systematic bias of different calibrations cannot be alleviated by getting deeper data. Comparisons between different calibrators and more detailed theoretical modeling are needed to resolve their discrepancies.

\subsection{Stellar Population Gradients}

Our science requirement on stellar populations is to measure the age, metallicity, and abundance gradients in quiescent galaxies and age gradients in star-forming galaxies to better than 0.1 dex per decade in $R_{e}$.

For each galaxy observed in the first year, using the data cube produce by the DRP, we Voronoi-bin the spaxels to have 

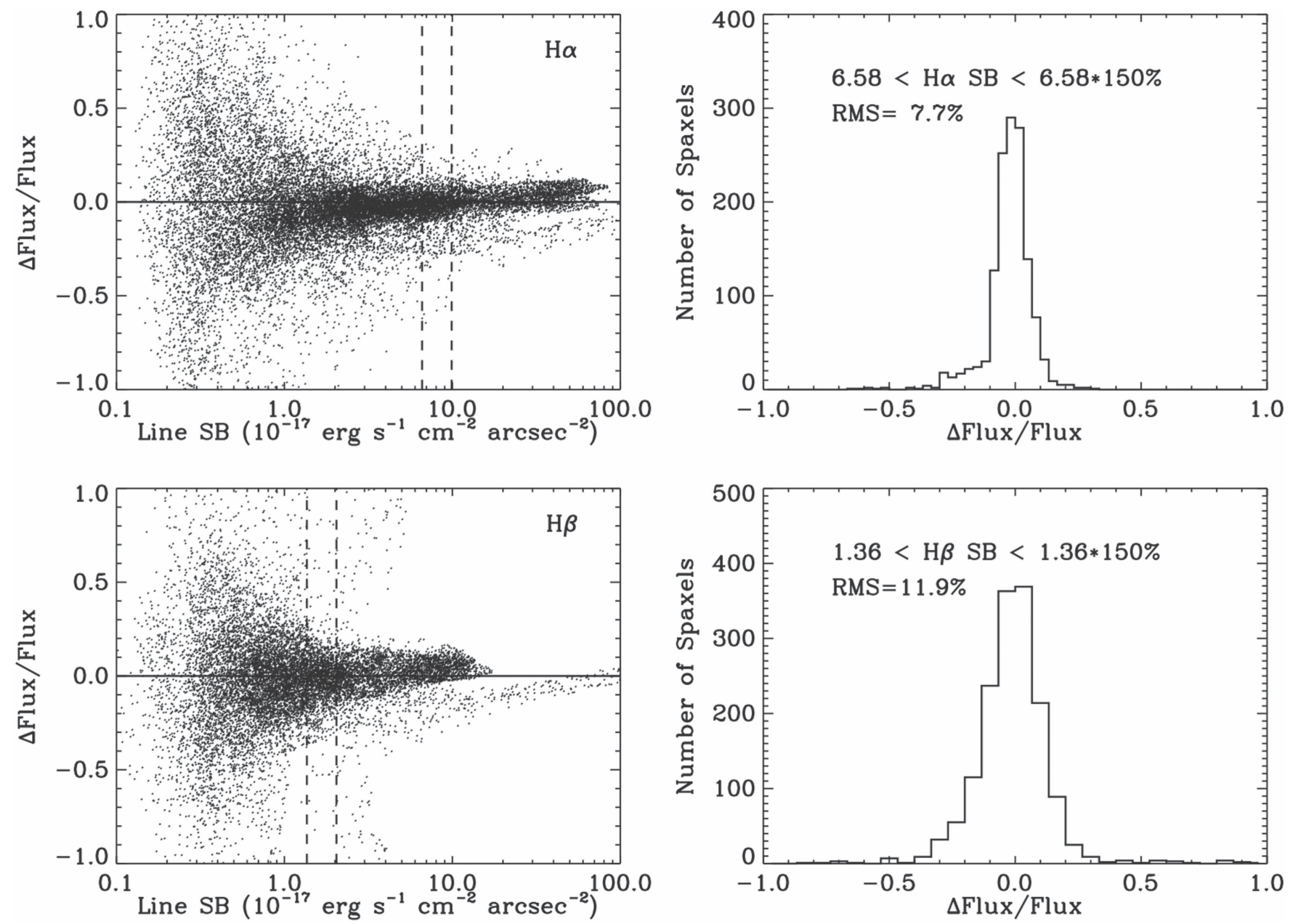

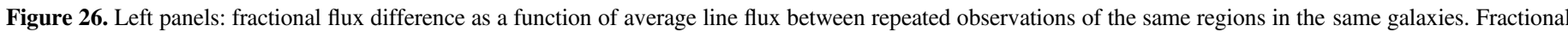

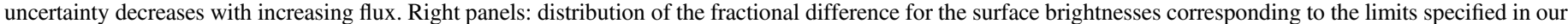

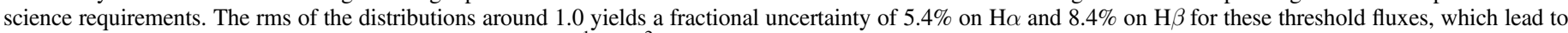
0.1 dex uncertainty on SFR surface density for $0.01 M_{\odot} \mathrm{yr}^{-1} \mathrm{kpc}^{-2}$ with an extinction $E(B-V)=0.5$.

$\mathrm{S} / \mathrm{N}$ greater than 5 per bin. We measured the stellar age and metallicity for each Voronoi bin and then fit the radial gradient with a linear function. We evaluate the uncertainty of the gradients using a Monte Carlo bootstrap resampling method. From a 1000 resampling of the original distribution for each galaxy, we measure the error on the slope. Figure 28 shows the uncertainty distribution of the stellar age and metallicity gradients for all early-type galaxies in the first-year data and that of the age gradient for late-type galaxies. The measurement will be described in D. Goddard et al. (2016, in preparation). We meet the stellar population gradient requirement for $\gtrsim 70 \%$ of early-type and late-type galaxies.

In Section 2, where we derived the $\mathrm{S} / \mathrm{N}$ needed to meet the science requirement, we assumed that we will make the measurement by stacking all spaxels within an annulus and produce one measurement per annulus with an associated uncertainty. In reality, such an approach would likely underestimate the uncertainty. There are two reasons. First, it does not include any intrinsic physical variation within an annulus. Second, whatever algorithm we use to estimate error for one data point may not be robust. It is much more reliable to measure the concerned quantity in many Voronoi bins within an annulus and then estimate the scatter among them. This scatter would include both the intrinsic scatter and the actual measurement uncertainty. The error on the final gradient derived from this would be much more robust.

\subsection{Specific Angular Momentum}

We require the specific angular momentum within $1 R_{e}$ to be measured to better than 0.05 around $\lambda_{R}=0.1$ so that we could distinguish fast and slow rotators.

We have measured $\lambda_{R_{e}}$ for all galaxies observed in the first year (M. Graham et al. 2016, in preparation). We estimated the uncertainty on $\lambda_{R_{e}}$ by generating random normal distributons for both velocity and velocity dispersion according to the measurement errors on them. We generated 100 pairs of these random kinematic maps and computed $\lambda_{R_{e}}$ for each. The uncertainty on $\lambda_{R_{e}}$ is derived by taking the standard deviation among them.

Figure 29 shows the uncertainty of $\lambda_{R_{e}}$ as a function of $\lambda_{R_{e}}$. Around $\lambda_{R_{e}}$ of 0.1 , we can see that nearly all galaxies have uncertainty better than 0.05 . This meets our requirement. However, in this calculation, we have not considered systematics due to the beam-smearing effect. This would need to be assessed by simulations and will be addressed in future work. 


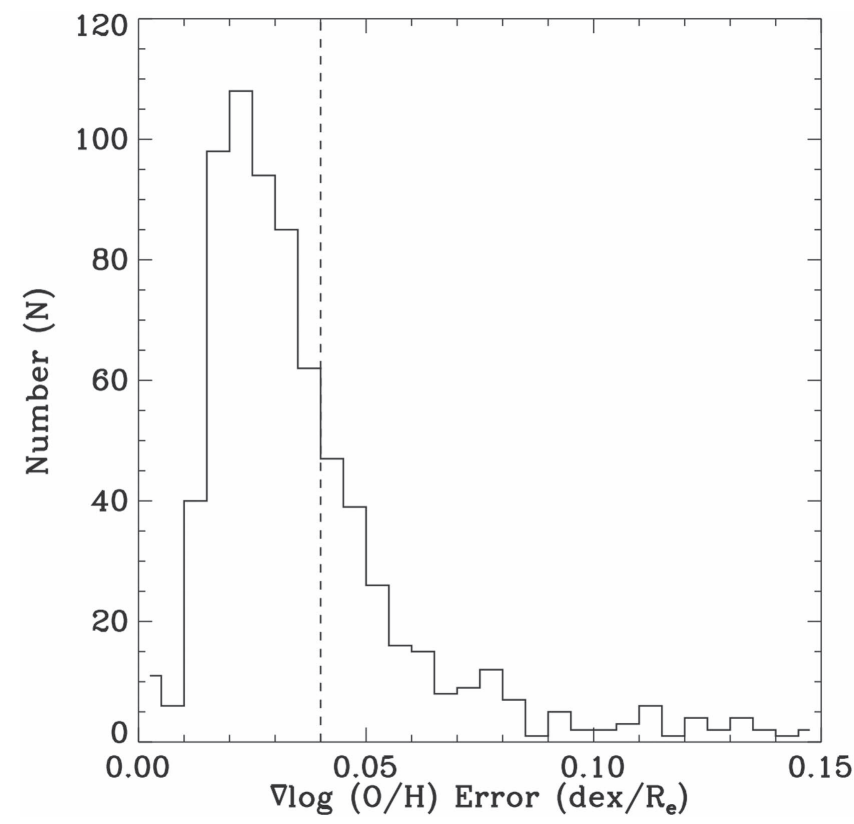

Figure 27. Uncertainty distribution of the gas metallicity gradient in all starforming galaxies observed in the first year. This is measured using the R23 metallicity indicator and the analytic formula given by Tremonti et al. (2004). The vertical line marks the threshold of the science requirement.

\subsection{Enclosed Gravitating Mass and Dark Matter Fraction}

In this section, we evaluate whether we meet the $10 \%$ accuracy requirement on the enclosed gravitating mass for all axisymmetric galaxies and the $10 \%$ precision requirement on the dark matter fraction within $1.5 R_{e}$ for early-type galaxies. We first address the gravitating mass requirement on rotationdominated disk galaxies, and then we address this and the dark matter requirement on early-type galaxies.

For rotation-dominated disk galaxies, we estimate the enclosed mass using the gas rotational velocity. The uncertainty is dominated by the error in the inclination. Compared to inclination error, the fractional error on the gas velocity is much smaller. We can measure the inclination from either photometry or kinematics. The difference between the photometric and kinematic inclinations can provide an indication of the uncertainty, which is typically much larger than the formal error provided by either measurement. To assess this, we select all rotation-dominated galaxies from the first-year observations that have stellar line-of-sight velocity more than twice as large as the stellar line-of-sight velocity dispersion at $1 R_{e}$ and have kinematic inclination between $15^{\circ}$ and $75^{\circ}$. This yields a subsample of 361 galaxies. Using the difference between photometric and kinematic inclination to derive the error on inclination $(1 / \sqrt{2}$ of the difference), we obtain the fractional uncertainty on enclosed mass according to the following formula:

$$
\frac{\Delta M}{M} \simeq \sqrt{2} \frac{\Delta \sin i}{\sin i}=2 \frac{\left(\sin i_{\mathrm{phot}}-\sin i_{\mathrm{kin}}\right)}{\left(\sin i_{\mathrm{phot}}+\sin i_{\mathrm{kin}}\right)} .
$$

Figure 30 shows the fractional uncertainty on mass as a function of kinematic inclination. The mean fractional errors (marked by the solid line) in bins of inclination indicate the systematic errors of the dynamical mass estimates; the standard deviations (marked by the error bar) indicate the random errors
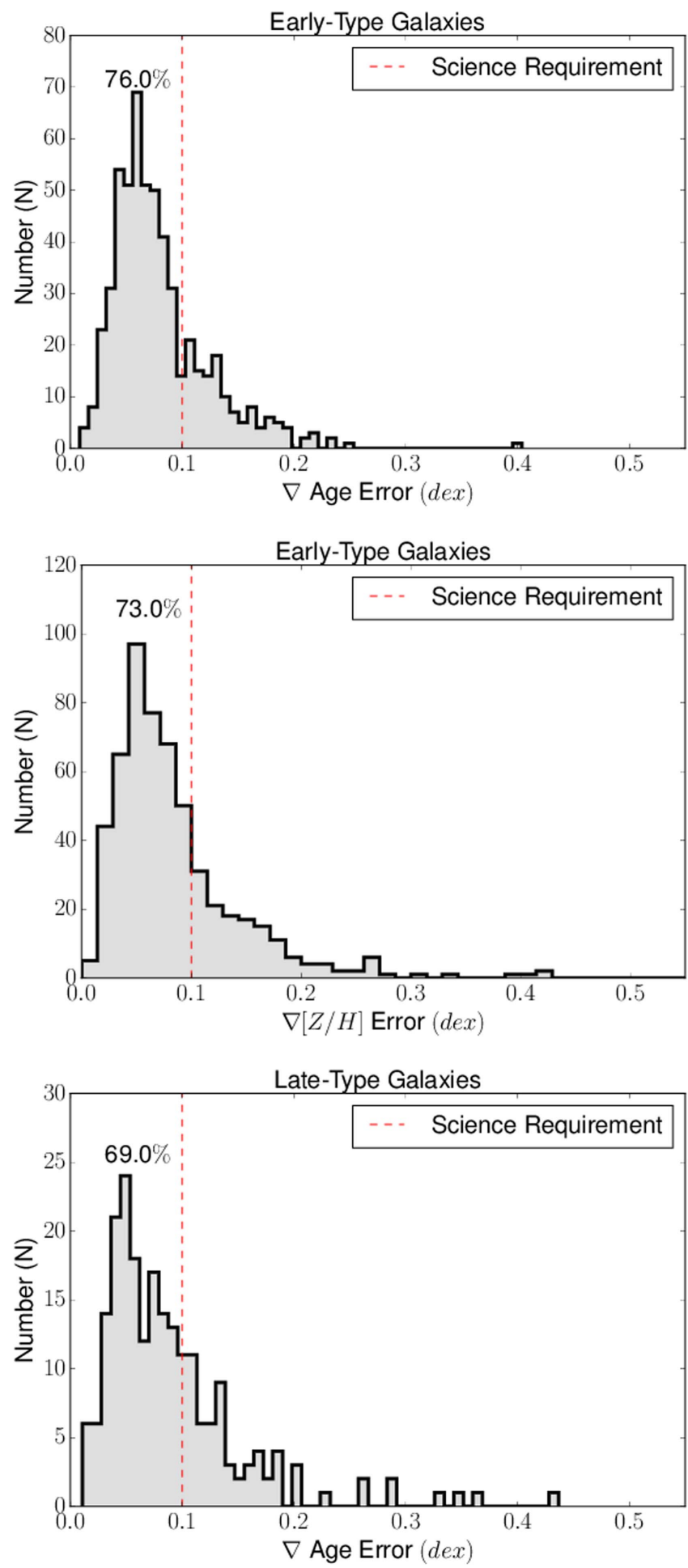

Figure 28. Uncertainty distributions of the stellar age gradient (top panel) and the metallicity gradient (middle panel) among early-type galaxies observed in the first year. The bottom panel shows the uncertainty distribution for the age gradient in late-type galaxies. The units of the gradients are per dex per decade in $R_{e}$. The vertical lines mark our science requirements, which are met by the great majority of galaxies.

for individual galaxies. The systematic errors are better than $10 \%$ in all bins, and the random errors are better than $10 \%$ at inclinations above $55^{\circ}$. Overall, we expect $62 \%$ of the sample to have a fractional error less than $10 \%$. 


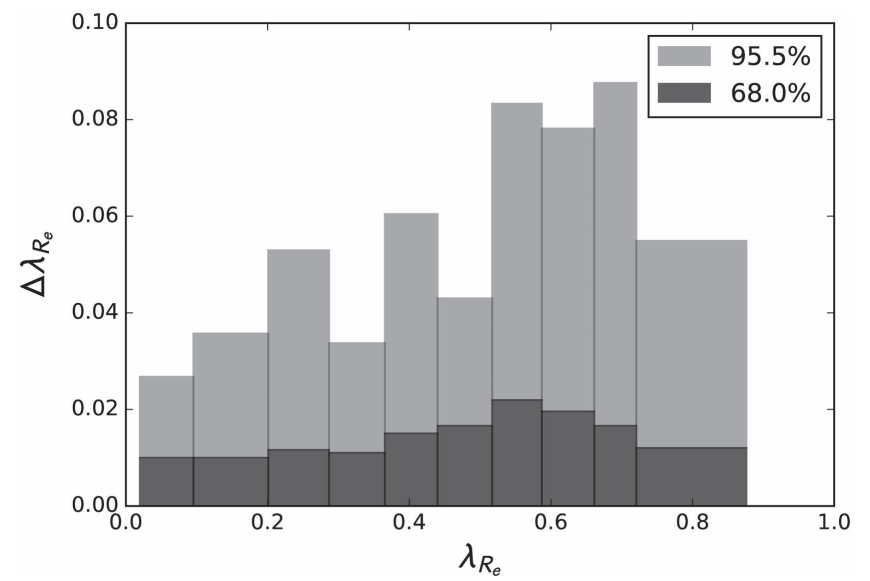

Figure 29. Distribution of the uncertainty on the specific angular momenum $\left(\Delta \lambda_{R_{e}}\right)$ as a function of $\lambda_{R_{e}}$. The black histogram shows the 68th percentile in $\Delta \lambda_{R_{e}}$ in each bin of $\lambda_{R_{e}}$. The gray histograms show the 95th percentiles.

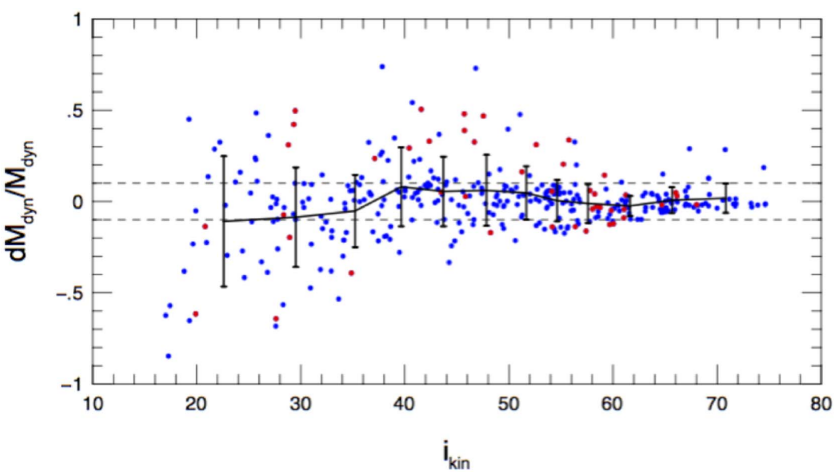

Figure 30. Fractional uncertainty on enclosed gravitating mass within $1.5 R_{e}$ as a function of the kinematic inclination, for all rotation-dominated galaxies observed by MaNGA in the first year. The blue and red points indicate blue and red galaxies, respectively. The solid line with error bars marks the mean values and standard deviation in bins of inclination. The error is dominated by uncertainty on derived inclination, which is estimated from the difference between photometric inclination and kinematic inclination. Adding the measurement errors of the gas or stellar velocity makes little difference to the results. The horizontal lines mark the science requirements. The majority of the objects in our sample satisfy this requirement.

For early-type galaxies, we estimate the enclosed gravitating mass and dark matter fraction in a different way. With the firstyear data, H. Li et al. (2016, in preparation) applied JAM to derive the dynamical mass estimate for all elliptical galaxies. The sample is defined by Galaxy Zoo classification being "elliptical," or by Sérsic index greater than 2.5 and de Vaucoulers fraction greater than 0.8 when Galaxy Zoo classification is "uncertain." Among 562 elliptical galaxies observed in the first year, 160 are rejected due to one of four reasons: many pixels having unphysical velocity dispersion (38), having fewer than 20 Voronoi bins (51), having a foreground star (12), or being a merger or in a close pair (59). Among the remaining 402 galaxies, we run JAM within a Markov chain Monte Carlo (MCMC) framework, as described by $\mathrm{Li}$ et al. (2016). From these, we estimated the statistical uncertainty on the enclosed gravitating mass and the dark matter fraction, using the 1D marginalized MCMC distributions. The distributions for these uncertainties are shown in Figure 31. About $85 \%$ of these 402 galaxies have a fractional error on total enclosed gravitating mass less than $10 \%$, and $72 \%$ have a dark matter fraction error less than $10 \%$.
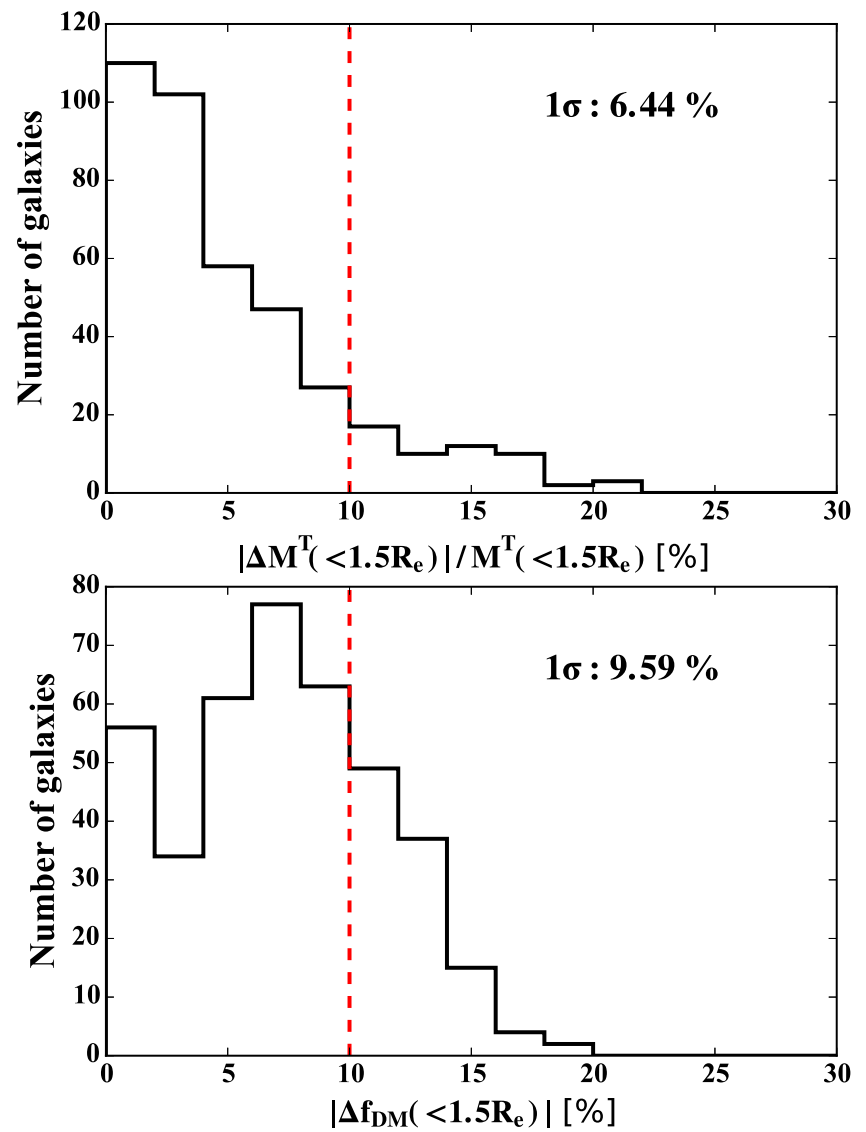

Figure 31. Top: distribution of the fractional error on enclosed gravitating mass within $1.5 R_{e}$ for early-type galaxies. Bottom: distribution of the error on dark matter fraction within $1.5 R_{e}$ for early-type galaxies. The vertical lines mark the science requirements. These errors are derived from the 1D marginalized MCMC distrubtion. They do not include the much larger systematic uncertainty associated with the JAM method. See text for details.

The overwheliming majority of the spaxels in most of these galaxies have velocity dispersion significantly above our instrumental resolution; thus, the velocity and velocity dispersion are reliably measured.

However, these errors are only relevant for the precision of the estimates, which is what we defined in the science requirements. In light of the recent work by $\mathrm{Li}$ et al. (2016), it is apparent that these random errors are dwarfed by the systematic error associated with the JAM method. Li et al. (2016) evaluated the accuracy of the JAM method using simulated galaxies from the Illustrius project (Genel et al. 2014; Nelson et al. 2015). They found that, with a $0.5 \mathrm{kpc}$ imaging resolution and $2 \mathrm{kpc}$ velocity field resolution, the true fractional uncertainty is $11 \%-16 \%$ on the total mass and $~ 33 \%$ on the dark matter mass within $2.5 R_{e}$, with relatively little bias (3\%) in the median value. If one degrades the imaging resolution to $2 \mathrm{kpc}$, there will also be a $10 \%$ systematic bias in the median value of the measured dark matter mass. Going for smaller radius may also result in worse uncertainty. This large systematic error is likely due to the simplified assumptions made in the JAM method, such as oblateness, constant $M / L$, constant anisotropy in the meridional plane, and a double power-law dark matter profile. These assumptions could fail for a significant fraction of galaxies.

Therefore, although it appears that we have met the science requirements on these quantities, currently it is unclear whether 
we can reach our science goal of measuring the stellar $M / L$ to better than $25 \%$ in order to improve the constraints on the IMF. Further simulations done with the actual MaNGA resolution for both $1.5 R_{e}$ and $2.5 R_{e}$ spatial coverage will provide the answers. In addition, the accuracy of the measurements is significantly better when higher-resolution imaging is available. Therefore, we can do significantly better in areas overlapping with HSC deep imaging fields.

\section{SUMMARY}

MaNGA is an integral field spectroscopic survey of 10,000 nearby galaxies with wide wavelength coverage at medium resolution with uniform spatial coverage in units of $R_{e}$. Up to the time of writing, we have already obtained observations for more than 2550 galaxies and are on track to finish $\sim 10,000$ by the summer of 2020 .

In this paper, we have detailed the survey science requirements, in terms of both random and systematic error$\mathrm{s}$ and how the high-level science requirements flow down in an interconnected way to the low-level requirements on the hardware, sample selection, observations, and analysis. In this context we have described in detail how the sample selection is carried forward to generate a survey footprint on the sky, how this footprint is parsed into tiles, how these tiles are targeted with plates, and how these plates are designed, fabricated, and scheduled for observation. The observing procedures are likewise detailed at a level necessary for a complete and reliable reconstruction of the survey execution. Finally, as proof of practice, we have given a complete demonstration of the data quality in both basic data products and high-level derived science products across the full first year of data.

The basic data quality of the survey is excellent. We have reached the $\mathrm{S} / \mathrm{N}$ target while staying on track to finish observing 10,000 galaxies by 2020 . We obtain a spatial resolution of about 2 ". 5 FWHM with a carefully characterized profile with uniform and near-critical sampling from multiple dithered observations. The sky subtraction is nearly Poisson even at near-IR wavelengths. Both the absolute and relative flux calibrations are better than 5\%. The spectral resolution is a function of wavelength and is characterized for each fiber in each exposure. Exposure-to-exposure variations should be taken into account if the science case warrants it.

The high-level derived science products are also of high quality. We have met the majority of the science requirements set forth, such as the precision on the SFR surface density, the gas metallicity gradient, the stellar population age, and the metallicity gradient. On the several kinematics requirements, such as the specific angular momentum, the enclosed mass, and the dark matter fraction, the systematic errors due to simplified modeling assumptions dominate the precision of the measurements. The formal errors appear to meet the science requirements, but whether the scienctific goals on kinematics could be reached awaits further analysis facilitated by detailed simulations. The first-year data will be released in SDSS Data Release 13 in summer 2016.

We thank the referee for a very constructive report that helped us to improve the paper. K.B. is supported by World Premier International Research Center Initiative (WPI Initiative), MEXT, Japan. M.A.B. acknowledges support by grant NSF/AST 1517007. A.W. acknowledges support of a Leverhulme Trust Early Career Fellowship. A.D. acknowledges support from the Grainger Foundation. D.B. acknowledges support by grant RSF 14-50-00043. M.C. acknowledges support from a Royal Society University Research Fellowship. S.M. and H.L. acknowledge support by the Strategic Priority Research Program "The Emergence of Cosmological Structures" of the Chinese Academy of Sciences Grant No. XDB09000000, and by the National Natural Science Foundation of China (NSFC) under grant number 11333003 and 11390372 (S.M.). K.M. acknowledges support by STFC.

Funding for the Sloan Digital Sky Survey IV has been provided by the Alfred P. Sloan Foundation, the U.S. Department of Energy Office of Science, and the Participating Institutions. SDSS-IV acknowledges support and resources from the Center for High-Performance Computing at the University of Utah. The SDSS Web site is http://www. sdss.org.

SDSS-IV is managed by the Astrophysical Research Consortium for the Participating Institutions of the SDSS Collaboration, including the Brazilian Participation Group, the Carnegie Institution for Science, Carnegie Mellon University, the Chilean Participation Group, the French Participation Group, Harvard-Smithsonian Center for Astrophysics, Instituto de Astrofísica de Canarias, Johns Hopkins University, Kavli Institute for the Physics and Mathematics of the Universe (IPMU)/University of Tokyo, Lawrence Berkeley National Laboratory, Leibniz Institut für Astrophysik Potsdam (AIP), Max-Planck-Institut für Astronomie (MPIA Heidelberg), MaxPlanck-Institut für Astrophysik (MPA Garching), Max-PlanckInstitut für Extraterrestrische Physik (MPE), National Astronomical Observatory of China, New Mexico State University, New York University, University of Notre Dame, Observatário Nacional/MCTI, The Ohio State University, Pennsylvania State University, Shanghai Astronomical Observatory, United Kingdom Participation Group, Universidad Nacional Autónoma de México, University of Arizona, University of Colorado Boulder, University of Oxford, University of Portsmouth, University of Utah, University of Virginia, University of Washington, University of Wisconsin, Vanderbilt University, and Yale University.

\section{REFERENCES}

Allen, J. T., Croom, S. M., Konstantopoulos, I. S., et al. 2015, MNRAS, 446, 1567

Andersen, D. R., Bershady, M. A., Sparke, L. S., et al. 2006, ApJS, 166, 505 Bacon, R., Copin, Y., Monnet, G., et al. 2001, MNRAS, 326, 23

Baldry, I. K., Glazebrook, K., Brinkmann, J., et al. 2004, ApJ, 600, 681

Beers, T. C., Flynn, K., \& Gebhardt, K. 1990, AJ, 100, 32

Belfiore, F., Maiolino, R., Maraston, C., et al. 2016, arXiv:1605.07189

Bernardi, M., Sheth, R. K., Annis, J., et al. 2003, AJ, 125, 1866

Bershady, M. A., Verheijen, M. A. W., Swaters, R. A., et al. 2010, ApJ, 716, 198

Best, P. N., Kauffmann, G., Heckman, T. M., et al. 2005, MNRAS, 362, 25

Bigiel, F., Leroy, A., Walter, F., et al. 2008, AJ, 136, 2846

Blanc, G. A., Weinzirl, T., Song, M., et al. 2013, AJ, 145, 138

Blanton, M. R., Eisenstein, D., Hogg, D. W., Schlegel, D. J., \& Brinkmann, J. 2005, ApJ, 629, 143

Blanton, M. R., Kazin, E., Muna, D., Weaver, B. A., \& Price-Whelan, A. 2011, AJ, 142,31

Blanton, M. R., \& Moustakas, J. 2009, ARA\&A, 47, 159

Blanton, M. R., \& Roweis, S. 2007, AJ, 133, 734

Brinchmann, J., Charlot, S., White, S. D. M., et al. 2004, MNRAS, 351, 1151 Bryant, J. J., Owers, M. S., Robotham, A. S. G., et al. 2015, MNRAS, 447, 2857

Bundy, K., Bershady, M. A., Law, D. R., et al. 2015, ApJ, 798, 7 Cano-Díaz, M., Sánchez, S. F., Zibetti, S., et al. 2016, arXiv:1602.02770 Cappellari, M. 2008, MNRAS, 390, 71 
Cappellari, M. 2016, arXiv:1602.04267

Cappellari, M., \& Copin, Y. 2003, MNRAS, 342, 345

Cappellari, M., Emsellem, E., Bacon, R., et al. 2007, MNRAS, 379, 418

Cappellari, M., Emsellem, E., Krajnović, D., et al. 2011, MNRAS, 413, 813

Cappellari, M., McDermid, R. M., Alatalo, K., et al. 2012, Natur, 484, 485

Cappellari, M., Scott, N., Alatalo, K., et al. 2013, MNRAS, 432, 1709

Cecil, G., Fogarty, L. M. R., Richards, S., et al. 2016, MNRAS, 456, 1299

Cenarro, A. J., Gorgas, J., Vazdekis, A., Cardiel, N., \& Peletier, R. F. 2003, MNRAS, 339, L12

Chen, X. Y., Liang, Y. C., Hammer, F., et al. 2010, A\&A, 515, A101 Colless, M., Dalton, G., Maddox, S., et al. 2001, MNRAS, 328, 1039

Conroy, C., \& van Dokkum, P. 2012a, ApJ, 747, 69

Conroy, C., \& van Dokkum, P. G. 2012b, ApJ, 760, 71

Cortese, L., Fogarty, L. M. R., Ho, I.-T., et al. 2014, ApJL, 795, L37

Croom, S. M., Lawrence, J. S., Bland-Hawthorn, J., et al. 2012, MNRAS, 421, 872

Dawson, K. S., Schlegel, D. J., Ahn, C. P., et al. 2013, AJ, 145, 10

Drory, N., MacDonald, N., Bershady, M. A., et al. 2015, AJ, 149, 77

Emsellem, E., Cappellari, M., Krajnović, D., et al. 2007, MNRAS, 379, 401

Emsellem, E., Cappellari, M., Krajnović, D., et al. 2011, MNRAS, 414, 888

Fogarty, L. M. R., Scott, N., Owers, M. S., et al. 2014, MNRAS, 443, 485

Fu, J., Kauffmann, G., Li, C., \& Guo, Q. 2012, MNRAS, 424, 2701

Genel, S., Vogelsberger, M., Springel, V., et al. 2014, MNRAS, 445, 175

Giovanelli, R., Haynes, M. P., Kent, B. R., et al. 2005, AJ, 130, 2598

Gomes, J. M., Papaderos, P., Kehrig, C., et al. 2016, A\&A, 588, A68

González Delgado, R. M., Cid Fernandes, R., García-Benito, R., et al. 2014, ApJL, 791, L16

González Delgado, R. M., Cid Fernandes, R., Pérez, E., et al. 2016, A\&A, 590, A44

González Delgado, R. M., García-Benito, R., Pérez, E., et al. 2015, A\&A, 581, A103

Gunn, J. E., Siegmund, W. A., Mannery, E. J., et al. 2006, AJ, 131, 2332

Ho, I.-T., Kewley, L. J., Dopita, M. A., et al. 2014, MNRAS, 444, 3894

Ho, I.-T., Medling, A. M., Bland-Hawthorn, J., et al. 2016, MNRAS, 457, 1257

Hogg, D. W., Blanton, M. R., Eisenstein, D. J., et al. 2003, ApJL, 585, L5

Johansson, J., Thomas, D., \& Maraston, C. 2012, MNRAS, 421, 1908

Kauffmann, G., Heckman, T. M., White, S. D. M., et al. 2003, MNRAS, 341,33

Kauffmann, G., Li, C., Zhang, W., \& Weinmann, S. 2013, MNRAS, 430, 1447

Kehrig, C., Monreal-Ibero, A., Papaderos, P., et al. 2012, A\&A, 540, A11

Kennicutt, R. C. 1998, ARA\&A, 36, 189

Kennicutt, R. C., \& Evans, N. J. 2012, ARA\&A, 50, 531

Kewley, L. J., \& Dopita, M. A. 2002, ApJS, 142, 35

Kewley, L. J., Dopita, M. A., Sutherland, R. S., Heisler, C. A., \& Trevena, J. 2001, ApJ, 556, 121
Kewley, L. J., \& Ellison, S. L. 2008, ApJ, 681, 1183

Koleva, M., Prugniel, P., de Rijcke, S., \& Zeilinger, W. W. 2011, MNRAS, 417, 1643

Kuntschner, H., Emsellem, E., Bacon, R., et al. 2010, MNRAS, 408, 97

Law, D. R., Cherinka, B., Yan, R., et al. 2016, AJ, arXiv:1607.08619

Law, D. R., Yan, R., Bershady, M. A., et al. 2015, AJ, 150, 19

Lawrence, A., Warren, S. J., Almaini, O., et al. 2007, MNRAS, 379, 1599

Li, H., Li, R., Mao, S., et al. 2016, MNRAS, 455, 3680

Mehlert, D., Thomas, D., Saglia, R. P., Bender, R., \& Wegner, G. 2003, A\&A, 407, 423

Moran, S. M., Heckman, T. M., Kauffmann, G., et al. 2012, ApJ, 745, 66

Nelson, D., Pillepich, A., Genel, S., et al. 2015, A\&C, 13, 12

Noeske, K. G., Weiner, B. J., Faber, S. M., et al. 2007, ApJL, 660, L43

Pérez, E., Cid Fernandes, R., González Delgado, R. M., et al. 2013, ApJL, 764, L1

Pettini, M., \& Pagel, B. E. J. 2004, MNRAS, 348, L59

Renzini, A., \& Peng, Y.-j. 2015, ApJL, 801, L29

Richards, S. N., Bryant, J. J., Croom, S. M., et al. 2016, MNRAS, 455, 2826

Salim, S., Charlot, S., Rich, R. M., et al. 2005, ApJL, 619, L39

Salim, S., Rich, R. M., Charlot, S., et al. 2007, ApJS, 173, 267

Sánchez, S. F., Kennicutt, R. C., Gil de Paz, A., et al. 2012, A\&A, 538, A8

Sánchez, S. F., Rosales-Ortega, F. F., Iglesias-Páramo, J., et al. 2014, A\&A, 563, A49

Sánchez-Blázquez, P., Rosales-Ortega, F. F., Méndez-Abreu, J., et al. 2014, A\&A, 570, A6

Schlegel, D. J., Finkbeiner, D. P., \& Davis, M. 1998, ApJ, 500, 525

Sharp, R., Allen, J. T., Fogarty, L. M. R., et al. 2015, MNRAS, 446, 1551

Singh, R., van de Ven, G., Jahnke, K., et al. 2013, A\&A, 558, A43

Smee, S. A., Gunn, J. E., Uomoto, A., et al. 2013, AJ, 146, 32

Spolaor, M., Kobayashi, C., Forbes, D. A., Couch, W. J., \& Hau, G. K. T. 2010, MNRAS, 408, 272

Stoughton, C., Lupton, R. H., Bernardi, M., et al. 2002, AJ, 123, 485

Strateva, I., Ivezić, Ž., Knapp, G. R., et al. 2001, AJ, 122, 1861

Thomas, D., Maraston, C., Schawinski, K., Sarzi, M., \& Silk, J. 2010, MNRAS, 404, 1775

Tremonti, C. A., Heckman, T. M., Kauffmann, G., et al. 2004, ApJ, 613, 898

Verheijen, M., Oosterloo, T., Heald, G., \& van Cappellen, W. 2009, in Panoramic Radio Astronomy: Wide-field 1-2 GHz Research on Galaxy Evolution, ed. G. Heald \& P. Serra, 10

Wilkinson, D. M., Maraston, C., Thomas, D., et al. 20152015, MNRAS, 449, 328

Wuyts, S., Förster Schreiber, N. M., van der Wel, A., et al. 2011, ApJ, 742, 96

Yan, R., Tremonti, C., Bershady, M. A., et al. 2016, AJ, 151, 8

York, D. G., Adelman, J., Anderson, J. E., et al. 2000, AJ, 120, 1579 